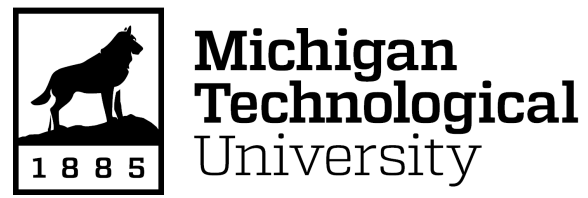

Michigan Technological University Digital Commons @ Michigan Tech

Dissertations, Master's Theses and Master's Reports

2019

\title{
A Smart Implantable Bone Fixation Plate Providing Actuation and Load Monitoring for Orthopedic Fracture Healing
}

Brad Nelson

Michigan Technological University, bnelson@mtu.edu

Copyright 2019 Brad Nelson

\section{Recommended Citation}

Nelson, Brad, "A Smart Implantable Bone Fixation Plate Providing Actuation and Load Monitoring for Orthopedic Fracture Healing", Open Access Dissertation, Michigan Technological University, 2019.

https://doi.org/10.37099/mtu.dc.etdr/898

Follow this and additional works at: https://digitalcommons.mtu.edu/etdr

Part of the Biomedical Devices and Instrumentation Commons 
A SMART IMPLANTABLE BONE FIXATION PLATE PROVIDING ACTUATION AND LOAD MONITORING FOR ORTHOPEDIC FRACTURE HEALING

By

Bradley D. Nelson

\begin{abstract}
A DISSERTATION
Submitted in partial fulfillment of the requirements for the degree of DOCTOR OF PHILOSOPHY

In Biomedical Engineering
\end{abstract}

MICHIGAN TECHNOLOGICAL UNIVERSITY

2019

(C) 2019 Bradley D. Nelson 
This dissertation has been approved in partial fulfillment of the requirements for the Degree of DOCTOR OF PHILOSOPHY in Biomedical Engineering.

Department of Biomedical Engineering

\author{
Dissertation Advisor: $\quad$ Keat Ghee Ong \\ Committee Member: Rupak Rajachar \\ Committee Member: Jingfeng Jiang \\ Committee Member: $\quad$ Parisa Pour Shahid Saeed Abadi \\ Department Chair: Sean J. Kirkpatrick
}




\section{Table of Contents}

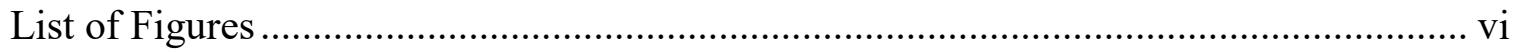

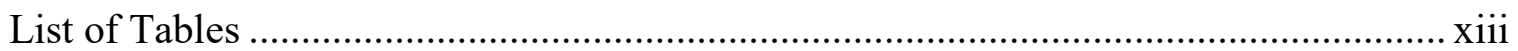

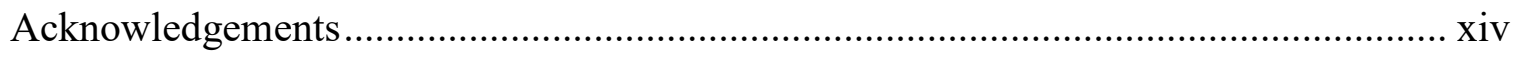

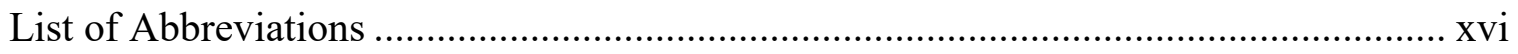

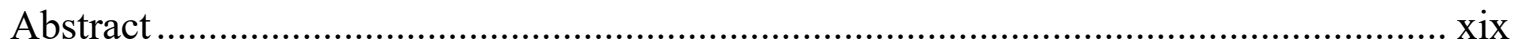

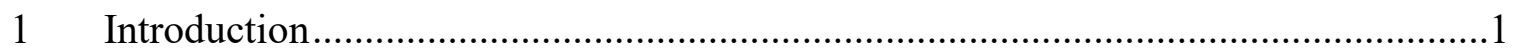

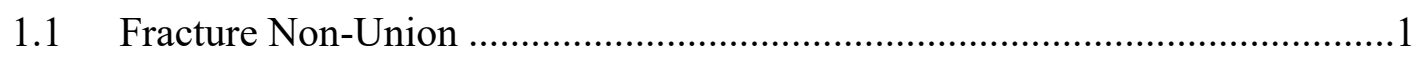

1.2 The Fracture Repair Process...................................................................2

1.3 Longitudinal Monitoring of Fracture Repair....................................................

1.4 Mechnobiology of Fracture Repair ..........................................................6

1.5 Current Treatment Methods ....................................................................

1.6 Project Scope

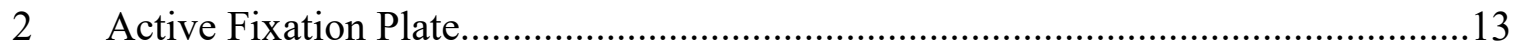

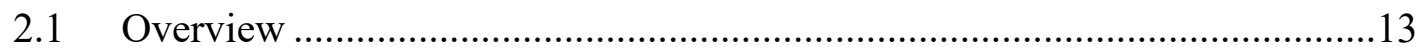

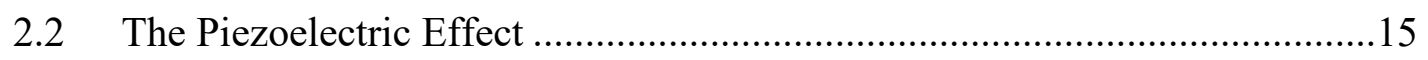

2.2.1 Mechanics of Piezoelectric Materials ..............................................16

2.2.2 Quantification of Piezoelectric Behavior..........................................18

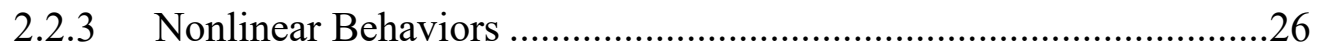

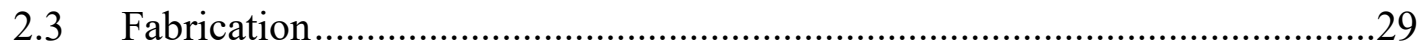

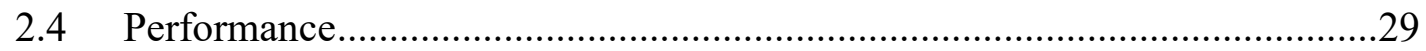

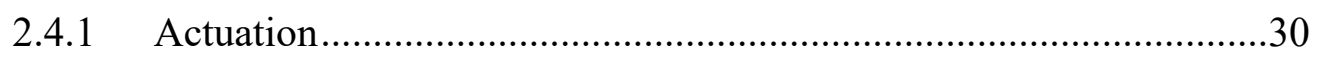




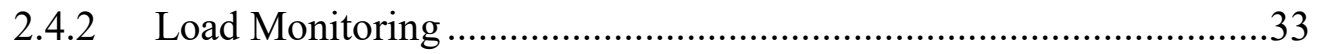

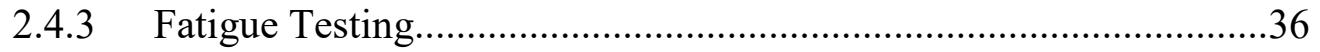

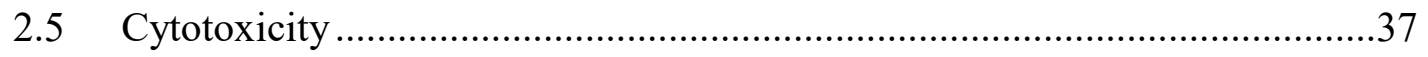

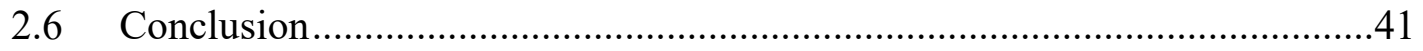

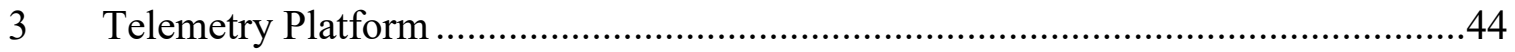

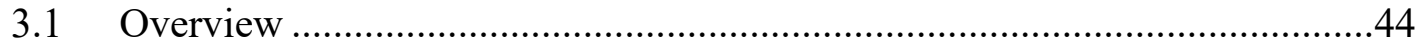

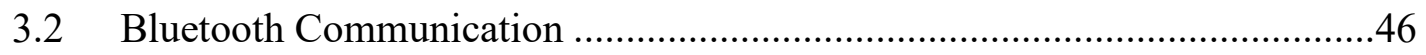

3.2.1 Pairing and Connectivity..............................................................46

3.2.2 Signal Propagation ............................................................................4

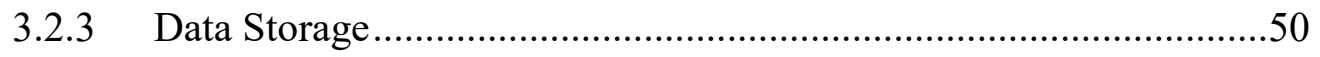

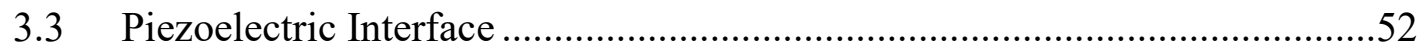

3.3.1 Actuation Pathway ....................................................................52

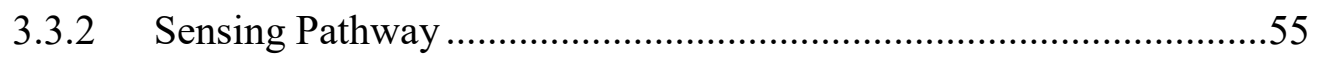

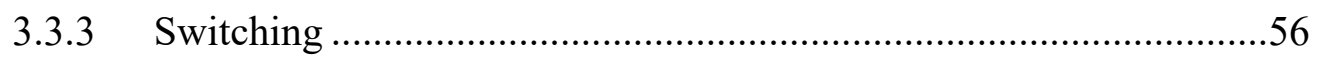

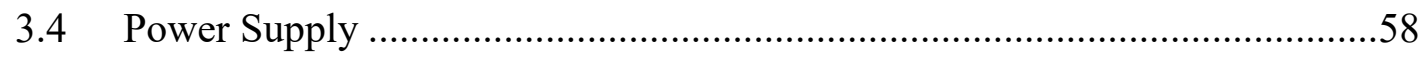

3.4.1 Wireless Power Transfer.............................................................58

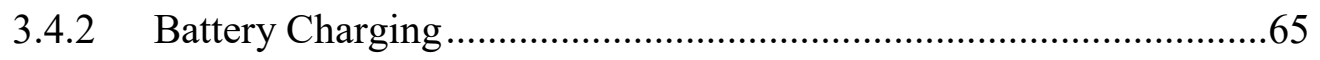

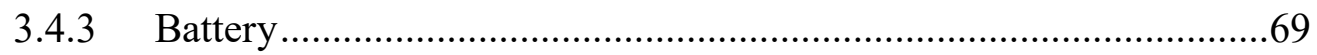

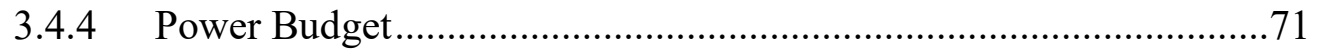

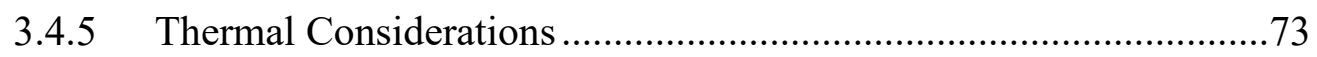

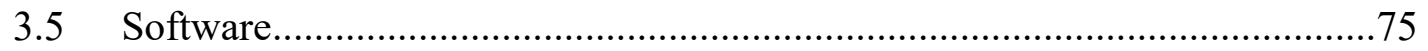

3.5.1 Implant Software ...........................................................................75

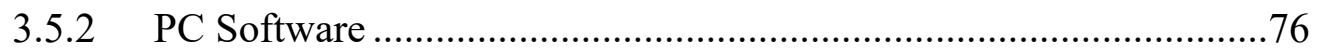

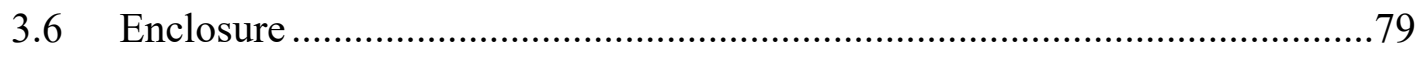

3.7 Electromagnetic Signal Propagation in Tissue...............................................81

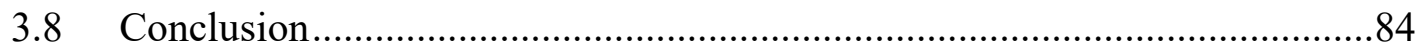




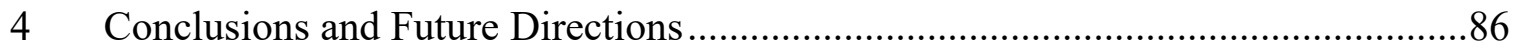

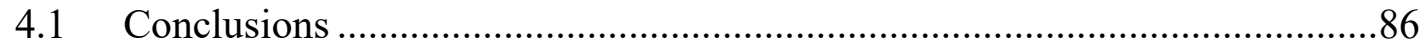

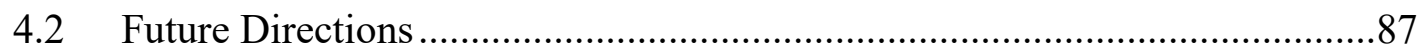

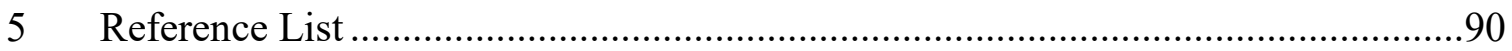

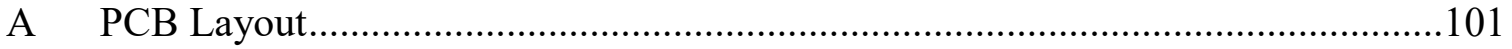

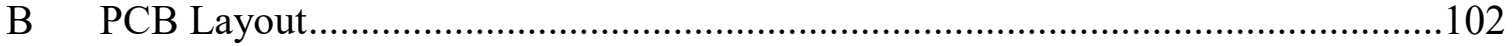

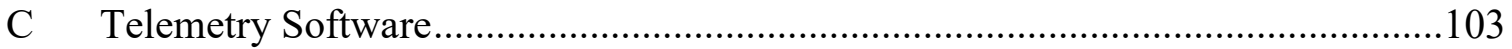

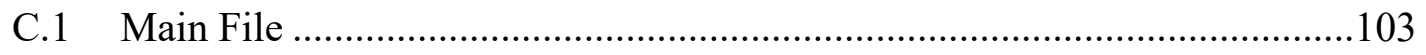

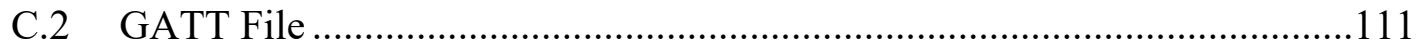

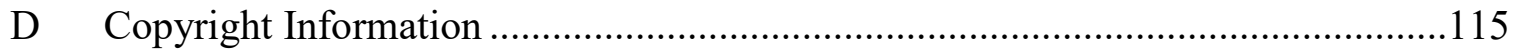




\section{List of Figures}

Figure 1.1 The major steps involved in endochondral ossification: (a) the formation of the hematoma, (b) the formation of a cartilaginous matrix, (c) the mineralization of the matrix, and (d) the remodeling of the callus area. ............................................2

Figure 1.2 Force diagram of the fixator-fracture system (a) before healing, where displacement $D=F /(E F+0)$; and (b) during healing, where displacement $D=$ $F /(E F+E C)$. As the stiffness of the callus increases, the overall displacement decreases. .5

Figure 1.3 Conceptualized plot of mechanical stimulus at the fracture site over the course of healing. Different magnitudes of mechanical stimulus promote the formation of different tissues. [36, 37], which quantify mechanical stimulus in terms of shear strain, shows that shear strains between 0.0375 and 0.1125 promote cartilage formation. Higher strains promote the formation of cartilage tissue, and lower strains promote bone formation

Figure 1.4 Model described in this work. The fixation plate is affixed across a $6 \mathrm{~mm}$ femoral defect and wired to the telemetry unit in the abdominal cavity. To quantify efficacy of treatment, a mesh containing a BMP-2 alginate is introduced to the fracture gap so that roughly $50 \%$ of fractures will heal without treatment

Figure 1.5 Complete implantable device consisting of a telemetry unit (left) wired to the fixation plate (right). The telemetry unit has dimensions 42.8 × 31.4 x $15.0 \mathrm{~mm}$. The fixation plate without riser plates has dimensions $5 \times 5 \times 25.6 \mathrm{~mm}$ .11

Figure 2.1 (a) Render and (b) photograph of the assembled piezoelectric fixation plate. The fixator dimensions without riser plates is $5 \times 5 \times 25.6 \mathrm{~mm}$, and the riser plates add an additional $2 \mathrm{~mm}$ in height. 
Figure 2.2 The unit cell of PZT. The black atoms (corners) represent $\mathrm{Pb}^{2+}$; the blue atoms (face centers) represent $\mathrm{O}^{2-}$; the red atom (body center) represents $\mathrm{Ti}^{4+}$ or $\mathrm{Zr}^{4+} \ldots 16$

Figure 2.3 Simplified behavior of a piezoelectric material, showing that $(a, b)$ applying stress to the material results in a separation of charge and (c, d) applying a voltage field across the material results in an induced strain. 17

Figure 2.4 Piezoelectric actuator featuring many interdigitated electrodes to create many smaller piezoelectric actuators within a single element. The PK2FMP2 used in this work has 59 effective layers in each chip. 18

Figure 2.5 Crystallographic directions used for describing piezoelectric axes. Along the Zaxis is direction 1, along the $\mathrm{Y}$-axis is direction 2, along the $\mathrm{X}$-axis is direction 3, around the $\mathrm{Z}$-axis is direction 4 , around the $\mathrm{Y}$-axis is direction 5 , and around the $\mathrm{X}$ axis is direction 6 .

Figure 2.6 Sample of creep behavior during the application of $15 \mathrm{~V}$ step to the actuator.

Figure 2.7 Sample of hysteresis present under cyclic actuation. The maximum hysteresis in this sample is approximately $10 \%$ of the maximum displacement .27

Figure 2.8 (a) Example of sensor drift during triangular loading in one of the more extreme cases. The average drift in this sample is $-1.7 \mathrm{mV} / \mathrm{s}$. The post-processed sample is shown in (b). .28

Figure 2.9 Mechanical testing configuration. Left: air gap to simulate an unbridged fracture. Center: rubber gap (stiffness $=12 \mathrm{MPa}$ ) to simulate cartilaginous callus. Right: Teflon gap (stiffness $=232 \mathrm{MPa}$ ) to simulate immature cortical bone........30

Figure 2.10 Actuation performance of the fixation plate (a) measured at a constant blocking force of $2 \mathrm{~N}$, (b) under different blocking forces from 2-10 N, and (c) showing how the actuation performance changes with blocking force. 31 
Figure 2.11 Actuation sensitivity with air, rubber, and Teflon gaps. The sensitivity in air is $124.9 \mathrm{~nm} / \mathrm{V}(\sigma=0.8 \mathrm{~nm} / \mathrm{V}, n=3)$, the sensitivity in rubber is $113.1 \mathrm{~nm} / \mathrm{V}(\sigma=1.0$ $\mathrm{nm} / \mathrm{V}, n=3)$, the sensitivity in Teflon is $40.8 \mathrm{~nm} / \mathrm{V}(\sigma=1.8 \mathrm{~nm} / \mathrm{V}, n=3)$.

Figure 2.12 Sample of the actuation profile of the fixation plate (amplitude $=0.7 \mu \mathrm{m}$, frequency $=31.25 \mathrm{~Hz}$ ).

Figure 2.13 Sensitivity to loading of the fixation plate without any gap material. (a) Intraplate sensitivity $(n=3)$ of $26.3 \mathrm{mV} / \mathrm{N}$; and (b) interplate sensitivity $(n=3)$ where the sensitivity ranges from $21.3 \mathrm{mV} / \mathrm{N}$ to $26.8 \mathrm{mV} / \mathrm{N}$. .34

Figure 2.14 Loading sensitivity in different materials. The sensitivity in air is $25.75 \mathrm{mV} / \mathrm{N}$ $(\sigma=0.33 \mathrm{mV} / \mathrm{N}, n=3)$, the sensitivity in rubber is $23.96 \mathrm{mV} / \mathrm{N}(\sigma=0.87 \mathrm{mV} / \mathrm{N}, n$ $=3)$, and the sensitivity in Teflon is $7.08 \mathrm{mV} / \mathrm{N}(\sigma=0.56 \mathrm{mV} / \mathrm{N}, n=3)$. .35

Figure 2.15 Voltage output of the fixation plate under sinusoidal compressive loading. The voltage difference measured when applying a $100 \mu \mathrm{m}$ load corresponds to an average of $339 \mathrm{mV}(\sigma=14 \mathrm{mV}, n=1)$. .35

Figure 2.16 Load sensing performance (a) before and (b) after 10,000 cycle fatigue test. There is no significant change in sensing performance between these two cases $(t$ $=-0.685, p=0.501)$.

Figure 2.17 Results of the cytotoxicity test at 24 and 72 hours at 10x magnification (inset at 4x magnification). Calcein-AM (green) shows live cells and ethidium homodimer-1 (red) shows dead cells.

Figure 2.18 Percentage of live cells relative to the number of total cells for media with and without exposure to fixator $(t=-2.230, p=0.050)$.

Figure 2.19 Fluorescence of cells exposed to fixators relative to the control media over (a) time exposed to fixators and over (b) time media is exposed to cells. (c) The average fluorescence of each plate relative to the control group. 
Figure 3.1 Block diagram showing the major functions of the telemetry platform described in this work.

Figure 3.2 (a) Unpaired central (C) and peripheral (P) devices. The peripheral device transmits packets until a connection is formed. When the devices are paired (b) they become the master $(\mathrm{M})$ and slave $(\mathrm{S})$ respectively.

Figure 3.3 Relative error rate of received signals at different distances in different media. Error rate is quantified as the byte error rate relative to the total packet length. There is much greater variance in error rate within trial conditions than between trial conditions .50

Figure 3.4 The GATT (Generic Attribute) profile used by the device described in this work.

Figure 3.5 Common topologies for switched DC/DC converters considered in this design:

(a) boost converter, (b) flyback converter, and (c) charge pump. .53

Figure 3.6 Actuation pathway. The TI DRV2667 generates voltages large enough to actuate the piezoelectric stack and can generate arbitrary waveforms .55

Figure 3.7 Schematic showing switching elements and their connections. .57

Figure 3.8 Loosely-coupled inductive wireless power transfer system. The transmitter coil used in this design is on the left; the receiver coil used in this design is on the right. A sinusoidal signal supplied to the transmitter coil generates a magnetic field. This magnetic field induces a sinusoidal current at the receiver coil.

Figure 3.9 Schematic of wireless power transmitter. The signal generated by a function generator is amplified before being transmitted by a coil inductor. 60 
Figure 3.10 Impedance spectrum of both (a) transmitter $(\mathrm{n}=3)$ and (b) receiver $(n=3)$ coils. Impedance significantly increases as the frequency approaches the resonant frequency for both coil designs.

Figure 3.11 Cross-sectional view of the simulated flux density of the magnetic field generated by the transmitter coil at $2 \mathrm{~A}$ current

Figure 3.12 Cross-sectional view of the simulated flux density of the magnetic field generated by the transmitter coil with ferrite shield at $2 \mathrm{~A}$ current.

Figure 3.13 Wireless power transfer efficiency through animal tissue showing (a) both vertical and horizontal effects of distance, (b) effects of displacement in the vertical direction at varying horizontal displacements, and (c) effects of displacement in the horizontal direction at varying vertical displacements.

Figure 3.14 Schematic for the rectification of the received power signal. Current is induced in the inductor by a nearby magnetic field. This AC current is then rectified using a diode bridge, capacitor, and zener diode

Figure 3.15 Schematic of the battery charger, charge sense, and battery level monitoring circuit.

Figure 3.16 Current received through wireless power transfer efficiency through animal tissue showing (a) both vertical and horizontal effects of distance, (b) effects of displacement in the vertical direction at varying horizontal displacements, and (c) effects of displacement in the horizontal direction at varying vertical displacements.

Figure 3.17 Battery voltage monitor profile $(n=3)$. The measured voltage $\left(\mathrm{V}_{\text {in }} / 2\right)$ is not isolated from the $\mathrm{ADC}$, so additional resistances pull the measurement away from the expected curve. Due to consistent behavior, the voltage monitor is simply characterized instead of introducing a voltage follower to the circuit. .68 
Figure 3.18 Schematic of the power supply for $2.5 \mathrm{~V}$ components

Figure 3.19 (a) Typical battery discharge profile for lithium polymer batteries. (b) Measured battery discharge curve for DTP502535. .70

Figure 3.20 (a) Typical battery charging profile for lithium polymer batteries. (b) Battery charging curve obtained using the DTP502535. .71

Figure 3.21 Maximum temperatures reached by the external transmitter coil $(n=3)$ as a function of supply current (RMS).

Figure 3.22 Port selection box. This is used to open serial communication with the BLED112 to enable Bluetooth communication. .76

Figure 3.23 List of discovered devices. The entry for Z1 has been expanded to reveal additional information including signal strength, bond, and a list of advertised information in hexadecimal format. .77

Figure 3.24 Table showing connected devices and the most recent data that has been received from them. .78

Figure 3.25 Window allowing writing of values to the server. .78

Figure 3.26 3D printed enclosure (a) containing the device components, (b) in exploded view, and (c) as photograph. The bottom is the superficial side of the telemetry unit.

Figure 3.27 Fixator attached to explanted rat femur via screws. The defect size is 6 $\mathrm{mm}$. .82

Figure 3.28 Load monitored by the fixation plate in the animal cadaver during manual actuation of the femur. The forces measured are in the range of 4 to $12 \mathrm{~N}$. .83 
Figure 4.1 Potential future fixation plate design featuring machined endcaps. The removal of separate riser plates eliminates a common failure mode experienced in similar

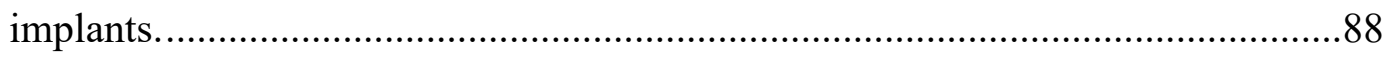




\section{List of Tables}

Table 2.1 Piezoelectric constants and their definitions [69]. $T$ is stress $\left(\mathrm{N} / \mathrm{m}^{2}\right), S$ is strain (dimensionless), $D$ is electric displacement $\left(\mathrm{C} / \mathrm{m}^{2}\right), E$ is electric field $(\mathrm{V} / \mathrm{m}) \ldots \ldots .19$

Table 2.2 Live/dead assay cell counts.............................................................................

Table 3.1 Approximate transmission range of the telemetry unit described in this work. In the water trial, the sensor was packaged and submerged in a container filled with tap water. In the tissue trial, the sensor was implanted in the cadaver of an adult Sprague-Dawley rat.

Table 3.2 Experimental average current and power consumption for the major subsystems

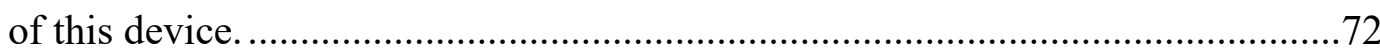




\section{Acknowledgements}

First of all, I would like to thank my advisor Prof. Keat Ghee Ong. His constant guidance along with his flexibility and care for his students have pushed me to succeed since my time as an undergraduate student. I would not be at this point without his constant encouragement to push myself.

I would also like to thank each member of my committee for their willingness to serve on my committee with their time and expertise.

Thank you to the members of the Ong lab for their assistance and support in this project, especially Salil Sidharthan Karipott, Deepak Jude Denny, Joe Stuck, and Cem Cedetas for their assistance in data collection. Also thanks to Srinivas Kannan for his assistance with the cell culture work.

I must thank Prof. Bob Guldberg and the members of his lab for their help getting our animal work off the ground. In particular, Brett Klosterhoff has been an invaluable support throughout this project thanks to his knowledge of the animal model. This project would still not be finished if I was not able to learn from him along the way.

I also thank Prof. Orhan Soykan for his support during my time as a teaching assistant and for his flexibility during long weeks of surgery.

Special thanks to Terry Major and Robert Bartlett's lab at the University of Michigan for the chance to learn from their surgical skills.

Additional thanks are due to Prof. George Muschler at the Cleveland Clinic for his orthopedic expertise.

Special thanks must go to my family and friends for their support in this long process as well as their eagerness to consistently pull me away from it. More people than I could possibly name have helped me more than they will ever know. 
This project would not have been possible without the financial assistance provided by the National Institute of Health (NIBIB grant 1R15 EB023648-10) and Portage Health Foundation.

Soli Deo Gloria. 


\section{List of Abbreviations}

3D Three dimensional

AC Alternating current

ADC Analog to digital converter

ANOVA Analysis of variance

AWG American wire gauge

BLE Bluetooth low-energy

BMP-2 Bone morphogenic protein 2

$\mathrm{CC} / \mathrm{CV}$ Constant current/constant voltage

CEM $43^{\circ} \mathrm{C}$ Cumulative effective minutes at $43^{\circ} \mathrm{C}$

CRC Cyclic redundancy check

CSV Comma separated value

DAC Digital to analog converter

DAQ Data acquisition device

DC Direct current

FBS Fetal bovine serum

GATT Generic attribute

GPIO General purpose input/output

I2C Inter-integrated circuit 
IC Integrated circuit

IEEE Institute of electrical and electronics engineers

ISM Industrial scientific medical

L2CAP Logical link control and adaptation protocol

LED Light emitting diode

LIPUS Low-intensity pulsed ultrasound

LMHF Low-magnitude high-frequency

MEM- $\alpha$ Minimum effective medium - alpha modification

MEMS Micro-electro-mechanical system

MOSFET Metal-oxide-semiconductor field-effect transistor

MSC Mesenchymal stem cell

PCB Printed circuit board

PCL Polycaprolactone

PFA Perfluoroalkoxy alkane

PTFE Polytetrafluoroethylene (Teflon)

PZT Lead zirconate titanate

RMS Root mean square

SD Sprague Dawley

SIG Special interest group

xvii 
USB Universal serial bus

UUID Universally unique identifier

WPF Windows presentation foundation

WPT Wireless power transfer

xviii 


\section{Abstract}

Fracture non-union occurs in roughly $5-10 \%$ of all fracture cases, and current interventions are both time-consuming and costly. There is therefore significant incentive to develop new tools to improve fracture healing outcomes. Several studies have shown that lowmagnitude, high-frequency (LMHF) mechanical loading can promote faster healing and reduce the risk of refracture in critical-size long bone fractures. This is typically done using whole-body vibration, which may result in undesirable systemic effects on the rest of the body. This work discusses an implantable piezoelectric fixation plate that can both apply LMHF loading directly to the fracture site using flexible scheduling and indirectly monitor the progress of healing by using the increasing stiffness of the fracture callus. The design and performance of the piezoelectric bone plate show that the device can apply the target treatment and has the sensitivity to be used to observe the progress of healing. An accompanying telemetry system using BLE communication is also introduced which has a footprint of suitable size to be used in rodent studies and can provide the power necessary for piezoelectric actuation. These results pave the way for future studies regarding the efficacy and optimization of LMHF treatments in fracture healing models. 


\section{Introduction}

\subsection{Fracture Non-Union}

Orthopedic fractures are common among all populations, with 12 to 15 million cases reported annually in the United States [1]. The most common treatments for fractures involve a combination of stabilization and time. This often takes the form of splints, casts, or various types of both internal and external fixation devices. In most cases, stabilized fractures are able to bridge and mineralize enough for the stabilization to be removed in about 8-16 weeks [2]. However, like most medical interventions, not all cases are resolved with equal success.

Fracture non-union - the failure of the bone to bridge properly during healing - is a pervasive problem that occurs in approximately $5-10 \%$ of reported fractures [3, 4]. The underlying cause of fracture non-union is not always known. A wide range of risk factors have been identified which are associated with increased occurrence of non-unions, such as osteoarthritis or the use of opioids [3,5]. However, these risk factors do little to help clinicians identify which fractures will result in non-unions.

For the $5-10 \%$ of patients that do not heal properly, additional interventions are needed in order for the fracture to heal. The cost of the additional interventions to successfully treat fracture non-unions is severe to patients, both in terms of inconvenience and finance. It is common for follow-up treatments to treat fracture non-union to take roughly 6 months, though they may continue for 2-3 years [6]. The cost of this process varies by method and country. A market review for the year 2006 found the median cost of non-union intervention in the U.S. was $\$ 25,556$ per patient, nearly $220 \%$ of the cost of the cost of a successful fracture healing without non-union [7]. A review published in the same year found the cost in the U.K. to be an average of $£ 29,204$ (approximately $\$ 51,234$ ) per patient [8]. Through current methods, non-union healing is both a lengthy and expensive process. 
It is very desirable to develop more effective tools and methodologies for the treatment of fracture non-unions to reduce both the personal and financial burdens on the patient. To develop these tools and methodologies, it is important to consider the mechanobiology of fracture repair and how it relates to existing treatment methods. Potential new methods of treatment may emerge that decrease the burden on both patient and physician by iterating on current treatments and research.

\subsection{The Fracture Repair Process}

There are two distinct fracture healing pathways which are often referred to as primary and secondary fracture healing [9]. Primary fracture healing-alternatively called intramembranous ossification-most commonly occurs in small fractures which experience limited strain. It is very rare for fractures of this nature to result in non-unions. Instead, large or complex fractures frequently heal through secondary fracture healing, also known as endochondral ossification [10]. This process is characterized by the formation and later mineralization of a fracture callus: a cartilaginous matrix between the bone fragments (Figure 1.1).

(a)

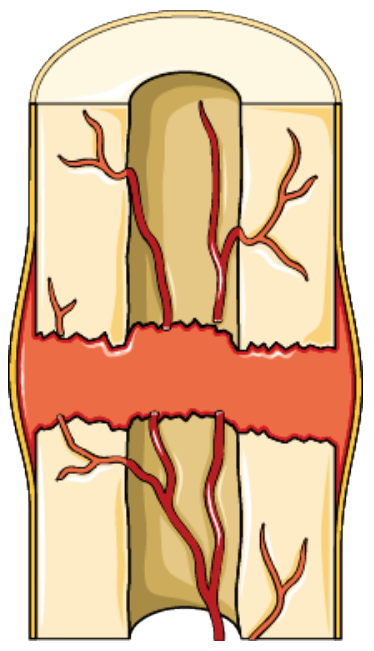

(b)

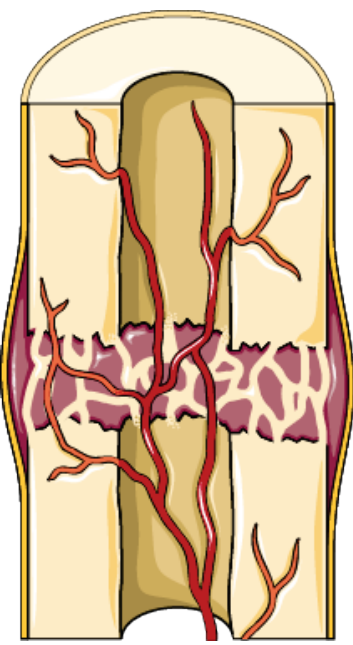

(c)

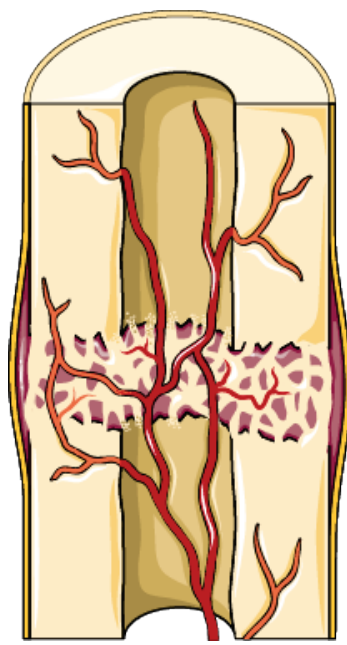

(d)

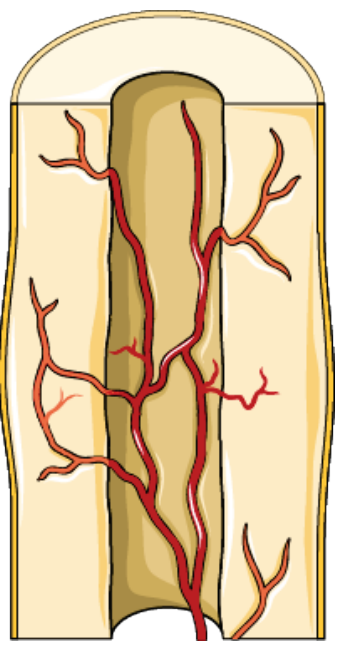

Figure 1.1 The major steps involved in endochondral ossification: (a) the formation of the hematoma, (b) the formation of a cartilaginous matrix, (c) the mineralization of the matrix, and (d) the remodeling of the callus area. 
The process of endochondral ossification is often described in a series of stages. Many authors use a three stage model containing an inflammation stage, healing phase, and remodeling phase [11]. Others use a four stage model which divides the repair phase into phases for the formation of the soft and hard callus [12,13]. While other models exist, such as those focusing on stages visible in radiography [14], the three stage model will be used to provide context for this work.

The first stage is often referred to as the inflammation phase. When the bone is fractured, the bone vasculature is ruptured along with it, resulting in an influx of blood and granulation tissue into the fracture cite. This forms the hematoma, which both provides a small degree of stabilization to the fracture and provides many immunological cells that can be used in the repair process. The hematoma is typically formed within the first 72 hours after the injury, but may take up to 2 weeks in rare cases [14].

During the repair stages, the fracture is bridged with a cartilaginous soft callus which is then replaced with a calcified hard callus. Typically, a significant amount of cartilage is present in the fracture gap by the middle of the second week [15], and the soft callus usually reaches its maximal size at 14 days [16]. After 14 days, the mineralization of the callus begins as the chondrocytes hypertrophy [2]. This mineralization turns the soft (or cartilaginous) callus into a hard (or bony) callus. Successful fracture healing is typically identified by a completed bridge of calcified tissue across the fracture gap. This typically occurs after 8-16 weeks [17].

After the callus material has been calcified, this woven bone matrix is remodeled into the structure of the surrounding bone. This process allows the damaged bone to return to the strength it had before the injury, which is particularly important for lamellar bone with a stronger axial strength. Post-fracture remodeling occurs at a faster rate than the normal remodeling experienced by uninjured tissue [18]. This remodeling process frequently takes years in humans, but can occur in less than 8 weeks in rodents [2]. 
Correctly identifying when a fracture is healed has important clinical implications. Patients who are considered to be healed prematurely and have their fixation devices removed are prone to refracture [19]. Additionally, speed of fracture healing is an important measurement in evaluating new fracture treatment methods. Radiography is commonly used to determine when a fracture has been healed completely. Using this method, a fracture is considered healed when the bridging of the fracture gap has been completely bridged with calcified matrix [2]. When possible, external fixation devices can be removed to more directly measure the stiffness of the bone [20]. For internal fixation devices, this is impossible without highly invasive surgery. Instead, wireless sensors are commonly used to indirectly monitor the progress of healing without surgical procedures [21].

\subsection{Longitudinal Monitoring of Fracture Repair}

Many "smart implants" have been developed for a wide range of applications by incorporating wireless sensors into orthopedic implants. Some such devices use strain gauges or load cells to better understand the mechanical forces acting on an implant during everyday activities [22-24], while others use sensors to detect worsening conditions such as spinal fusion [25]. Others have used sensors to measure the progress of fracture healing.

Knowledge of the strength of the healed bone provides valuable information to clinicians throughout the healing process. Additional interventions may become necessary if the fracture is not healing as expected, and incomplete healing leads to an increased risk of refracture [19]. It is common to use X-ray imaging to determine the size of the fracture callus during the fracture healing process. However, the size of the calcified callus alone does not indicate its mechanical properties [26]. The mechanical properties of externally fixated fractures can be determined using direct mechanical testing by removing the fixation device and measuring the force applied to the transcutaneous screws which is required to achieve a specified angle of deflection [20]. Instead, many have attached strain gauges to fixation plates to indirectly measure the stiffness of the fracture callus [27, 28]. As the stiffness of the callus increases, the measured strain decreases (Figure 1.2). 

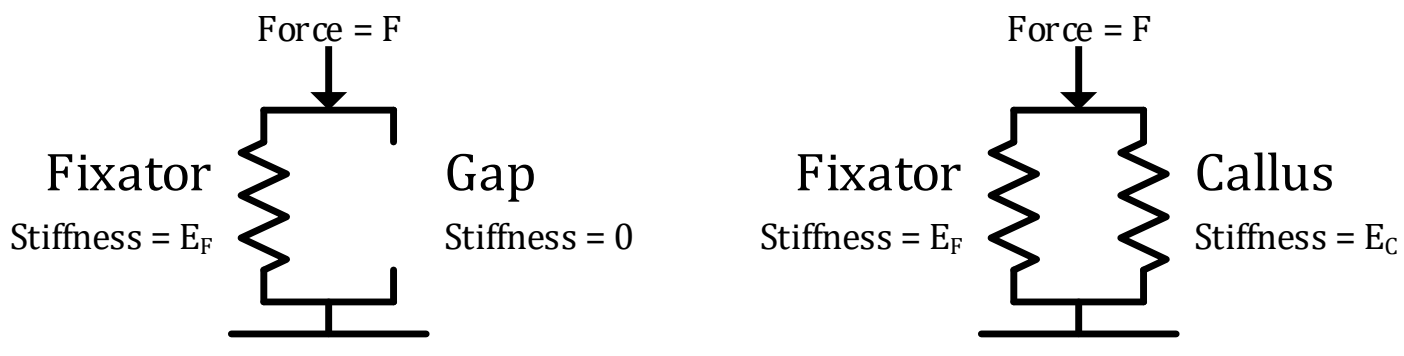

Figure 1.2 Force diagram of the fixator-fracture system (a) before healing, where displacement $D=F /\left(E_{F}+0\right)$; and (b) during healing, where displacement $D=$ $F /\left(E_{F}+E_{C}\right)$. As the stiffness of the callus increases, the overall displacement decreases.

Strain gauges have been implemented in fixation plates to observe longitudinal trends in bone stiffness throughout the fracture healing process [21, 29]. By tracking the relative changes in strain during gait, strain gauge measurements can be used to indicate relative changes in the callus stiffness. Although it is difficult to use current implantable sensorbased fixators to measure the absolute stiffness of the fracture callus, but the information it provides is still valuable in a clinical setting. For example, delayed stiffening may indicate delayed healing or non-union; increased strain can indicate a loosening of the fixation device or callus resorption.

Strain gauge-based stiffness data can also be used to evaluate the effectiveness of novel treatment methods throughout the healing process [29, 30]. Endpoint data, such as stiffness and vascularization measurements, are often supplemented with X-rays to obtain a better picture of the progress of healing over time. By incorporating strain-based measurements throughout the healing process, more direct measurements of healing progress may be taken at various time points to obtain a more complete picture of the mechanical features of the healing bone. 


\subsection{Mechnobiology of Fracture Repair}

It is well known that mechanical factors in the local fracture environment play a major role in fracture healing [31]. Large or complex fractures do not heal properly if the loads at the fracture site are too small [32]. It has long been understood that controlling the mechanical fracture environment can direct the course of healing. The stiffness of the fracture environment increases significantly over time in the presence of motion [33]. It may be noted that non-mechanical components are typically included with mechanical treatments [34]. For example, The addition of BMP-2 in an alginate to the fracture site has been shown to significantly promote bone formation [35].

The reason that the mechanical environment plays a critical role in fracture healing is because it is the major factor in the differentiation of mesenchymal stem cells into osteoblastic cells or fibroblasts [36]. Several models have been proposed to describe the relationship between forces such as strain and the generation of different tissue types during fracture healing [32, 37-39]. These theories propose that the stromal mesenchymal stem cells (MSC) present in the hematoma differentiate into different cells such as fibroblasts, chondroblasts, and osteoblasts depending on the mechanical environment. These differentiated cells also detect stresses and strains in their environment, and release growth factors to promote the formation of new bone and vascular tissues [40]. Factors including the timing [41], magnitude [42], and frequency [43] of mechanical stimulation have been shown to influence the speed and quality of fracture healing. 


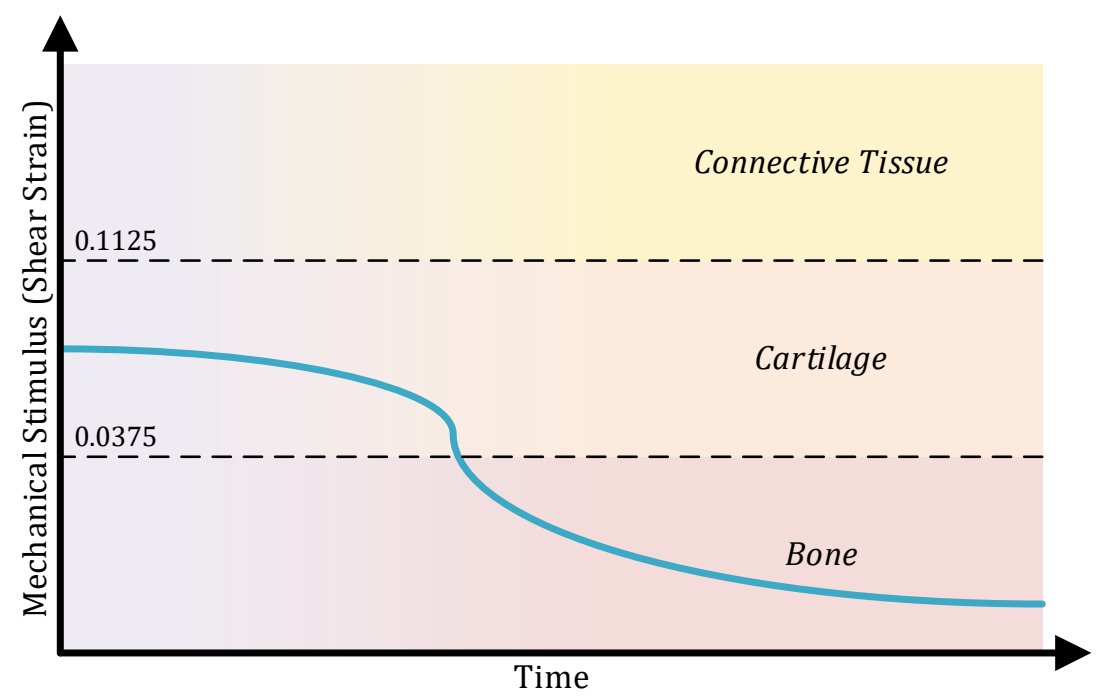

Figure 1.3 Conceptualized plot of mechanical stimulus at the fracture site over the course of healing. Different magnitudes of mechanical stimulus promote the formation of different tissues. [36, 37], which quantify mechanical stimulus in terms of shear strain, shows that shear strains between 0.0375 and 0.1125 promote cartilage formation. Higher strains promote the formation of cartilage tissue, and lower strains promote bone formation.

Figure 1.3 shows the generalized relationship of strain on resultant tissue. When high strain is induced in the fracture site, such as may occur without proper fixation, connective tissue forms which inhibits fracture healing. Moderate strains, such as those present in early stages of the endochondral ossification process, promote the creation of cartilage. This helps to form the soft callus during the repair phase. Low strains promote the formation of bone tissue. Bone is typically formed once the soft callus sufficiently stiffens the fracture site. Very low strains, such as those present in excessively rigid fixation, promote the resorption of bone. Based on this model, endochondral ossification can be promoted by applying moderate strains to the fracture site after the formation of the hematoma so that the cartilaginous soft callus forms successfully. Therefore, continuous monitoring of strain at the fracture site can be useful in inferring progress of healing and the strength of the newly formed bone. 


\subsection{Current Treatment Methods}

Several regimes of mechanical stimulation have been used in fracture healing. The traditional method for fracture healing is to incrementally increase the weight applied to the fractured bone over time [44]. Even with such established methods, 5-10\% of fracture treatments end in delayed or non-unions $[3,45]$. The cause is not always known.

The use of functional (or body weight) loading on fractures has been shown to improve healing speed [46]. Functional loading is especially common on load-bearing limbs such as legs. Partial weight bearing is often prescribed during the healing process [44]. During this treatment, the patient will incrementally increase the weight applied to the limb throughout the healing process until full body weight may be used. This treatment is typically difficult for patients to perform [47]. It is also possible to modulate the strains applied to the fracture site by modifying the compliance of a fixation plate. More compliant plates can increase the volume of bone formed in the fracture gap to improve healing outcomes [48]. Although mechanical loading has been known to be instrumental in the healing of bone fractures, research has shown that loading too early can result in slower healing [49].

Significantly lower strain magnitudes have been used in low-intensity pulsed ultrasound (LIPUS) treatments. This treatment method uses an external ultrasound generator to apply ultrasonic vibration through tissue to the target bone. The loading in this regime is typically defined by the power of the signal [50], but the effect in terms of displacement is expected to be on the order of nanometers [51]. The signal is commonly composed of megahertz pulses at kilohertz intervals [52]. LIPUS studies have showed promising results of decreased healing time and increased bone volume [53].

A particularly beneficial mechanical regime for fracture healing is the low-magnitude, high-frequency (LMHF) [54]. LMHF loading, which is typically between 20-90 Hz at less than $10 \%$ of the displacement experienced during ambulation, has shown faster healing and improvement to the mechanical properties of the healed bone $[43,55]$. However, most 
LMHF research uses external actuating fixation plates for larger animal models [43], or vibrating tables or platforms for small animals to shake the entire animal instead of the local area of treatment [55]. This makes the exact magnitude of the force applied to the fracture site difficult to identify. Furthermore, whole body vibration also introduces systemic effect on the test subject, thus LMHF still faces challenges before it can be translated clinically.

\subsection{Project Scope}

This overarching goal of this project is the development of a novel internal femoral fixation plate that can provide LMHF loading to a critical-sized rodent femoral defect [48], and monitor the strain of the fixation plate over time to observe the healing process (Figure 1.4). To evaluate improvements to fracture healing caused by mechanical treatment in a critical-sized defect, BMP-2 is commonly added to the fracture site. In these cases, a hollow cylindrical polycaprolactone (PCL) mesh can be inserted at the fracture site so that each end of the mesh covers bone near the fracture site. Once this mesh is in plate, an alginate containing BMP-2 may be injected into the mesh via a syringe. The amount of BMP-2 is such that roughly $50 \%$ of fractures will bridge without additional interventions so that the results of the investigation may be statistically analyzed. This dissertation focuses on the design, fabrication, and characterization of the internal femoral fixation plate. 


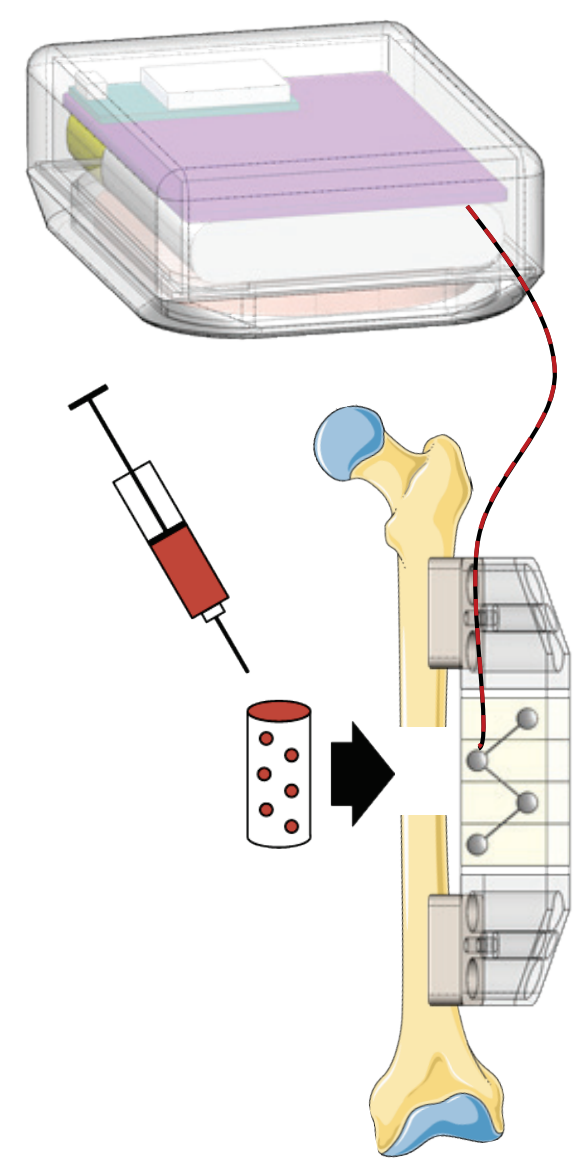

Figure 1.4 Model described in this work. The fixation plate is affixed across a $6 \mathrm{~mm}$ femoral defect and wired to the telemetry unit in the abdominal cavity. To quantify efficacy of treatment, a mesh containing a BMP-2 alginate is introduced to the fracture gap so that roughly $50 \%$ of fractures will heal without treatment.

The design of this device, shown in Figure 1.5, contains two main components: an internal fixation plate which both senses mechanical loading and applies tension to the fracture site at a range of magnitudes and frequencies, and a control unit which receives commands and transmits measurements wirelessly. The fixation plate is physically wired to the control unit, which is implanted inside the abdominal cavity of the test subject. This device will be used to both apply mechanical stimulation directly to the fracture site, as well as to monitor the resulting healing in real time. 


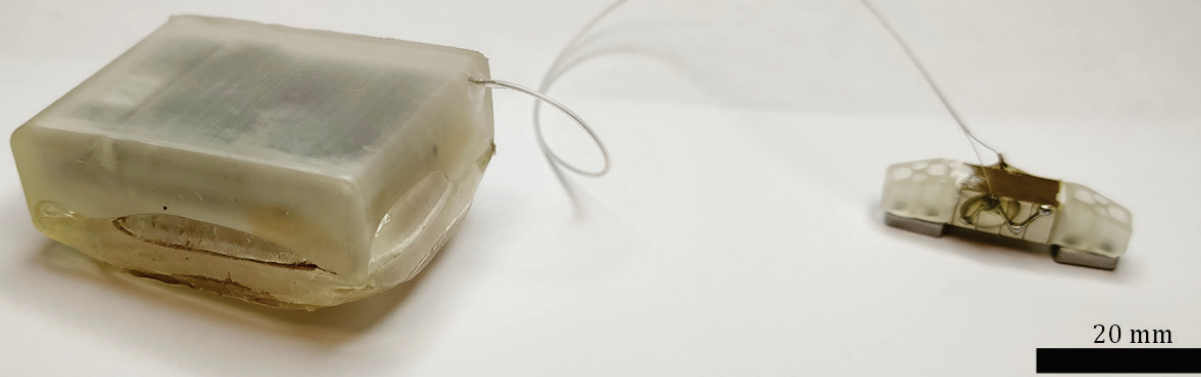

Figure 1.5 Complete implantable device consisting of a telemetry unit (left) wired to the fixation plate (right). The telemetry unit has dimensions $42.8 \times 31.4 \times 15.0$ $\mathrm{mm}$. The fixation plate without riser plates has dimensions $5 \times 5 \times 25.6 \mathrm{~mm}$.

This project has the following specific aims:

\section{Aim 1: Create an internal bone fixation plate with piezoelectric actuation and load monitoring for femoral fractures in rats.}

The design, fabrication, and mechanical testing of the active fixation plate is described in Chapter 2. The fixation plate will use a stack of piezoelectric elements to provide local actuation to the fracture site as well as to monitor loading experienced during ambulation.

Success in this aim will consist in demonstrating the ability of the fixation plate to both provide actuation in the LMHF range (displacement on the order of 0.1 to $10 \mu \mathrm{m}$ ) and monitor loading in the physiological range (loads on the order of roughly 1 to $15 \mathrm{~N}$ ). The loading sensitivity of the plate is expected to change measurably in off-axis loading tests when compressing materials of varying physiological stiffnesses (from no stiffness for unbridged calluses to roughly $10 \mathrm{MPa}$ for the soft callus and up to $1000 \mathrm{MPa}$ for immature bone) in order to monitor changes in bone stiffness. 


\section{Aim 2: Create a fully implantable telemetry control system to generate voltage signals to control actuation, to allow load monitoring of the piezoelectric material, and to allow wireless control and data collection.}

The design, fabrication, and characterization of the telemetry control system is described in Chapter 3. The telemetry unit will utilize Bluetooth Low-Energy (BLE) communication to control actuation and sensing, as well as to report data to an external computer. Circuit components and their functions will be described and characterized when appropriate.

Success in this aim will consist in generating voltage signals to the fixation plate that can generate LMHF loading (roughly 0.1 to $10 \mu \mathrm{m}$ displacements at roughly $35 \mathrm{~Hz}$ ). The control unit must be able to provide these signals continuously for at least 20 minutes at a time without draining the battery so that a typical LMHF loading treatment may be applied. The battery lifetime of the device must be able to provide power for a minimum of 8 weeks. A wirelessly rechargeable battery will be used to attain this. In addition, both the wireless telemetry and wireless charging systems must be able to operate through tissue when the device is implanted in an animal. 


\section{Active Fixation Plate}

\subsection{Overview}

Internal fixation plates have been used to treat fractures since the late $19^{\text {th }}$ century [56]. Rigid fixation methods have long been used to guide the fracture through intramembranous ossification. These plates are used to hold the fractured portions of bone in position and restrict movement during healing. This method is very reliable for small fractures, and is effective in larger fractures if the fracture gap can be reduced [57]. As a result, more flexible fixators are often preferred.

More compliant fixation has been used to guide fracture healing through the process of endochondral ossification. Methods such as those described by Ilizarov [58] have shown greater success at healing non-unions and other hard-to-treat fractures. These fixators allow for micromotion in the fracture site, promoting the formation of a cartilaginous matrix to bridge bone fragments before calcifying the resulting tissue.

Few examples of fixators that actively apply loading are present in the literature. One example of this uses Terfenol-D, a magnetostrictive material, to apply actuation using a magnetic field [43]. Active fixators may be used to apply and further investigate alternative loading regimes such as low-magnitude high-frequency (LMHF) vibration treatment.

The active element of the fixation plate (Figure 2.1) described in this work is the PK2FMP2 (ThorLabs, Newton, NJ, USA). This element is a stack of four piezoelectric chips made of a proprietary PZT (Lead Zirconate Titanate) material. A thin, ceramic plate is adhered to both ends of the piezoelectric stack to help provide a more uniform loading distribution. Electrodes are exposed on both sides of the stack so wires may be attached to provide actuation or sensing behavior. 
(a)

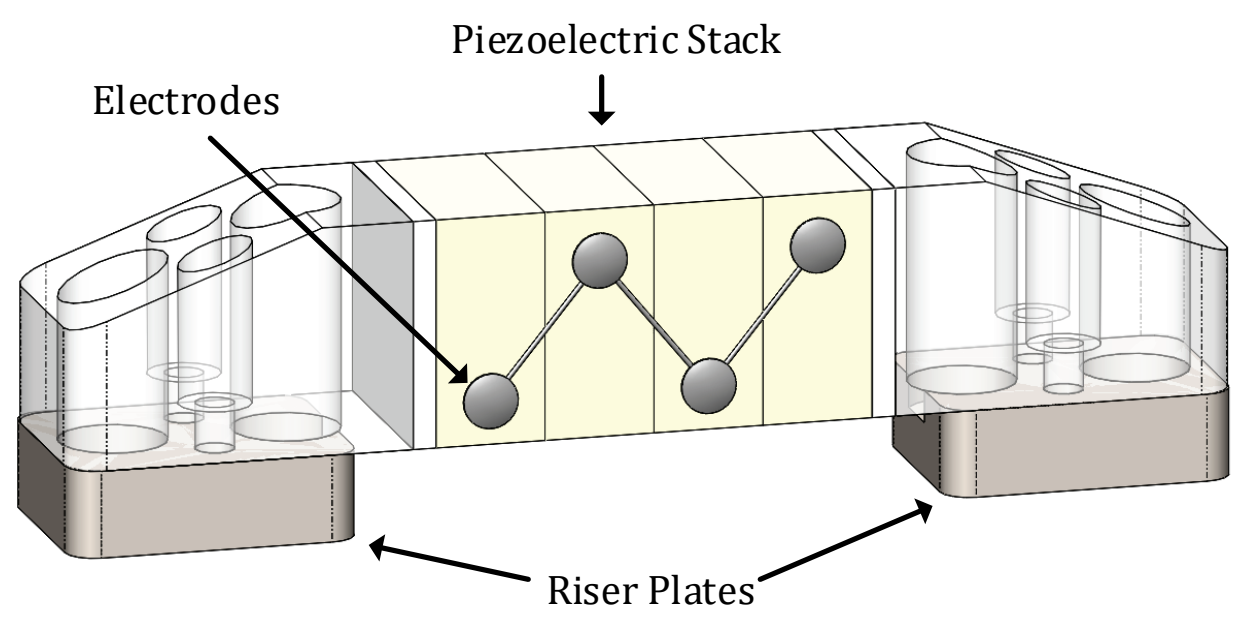

(b)

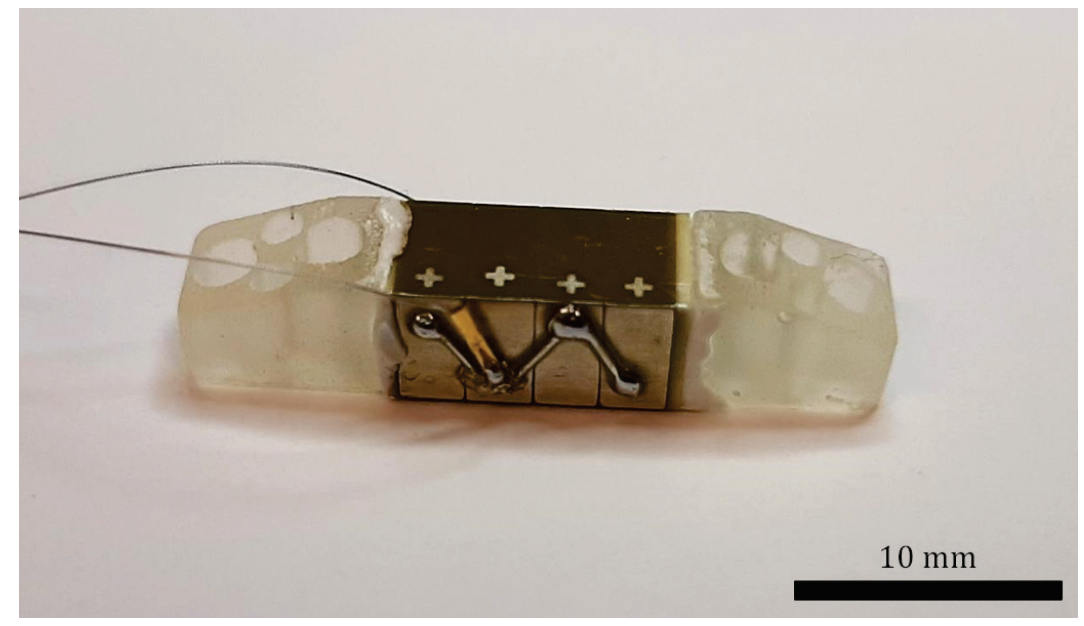

Figure 2.1 (a) Render and (b) photograph of the assembled piezoelectric fixation plate. The fixator dimensions without riser plates is $5 \times 5 \times 25.6 \mathrm{~mm}$, and the riser plates add an additional $2 \mathrm{~mm}$ in height.

3D-printed endcaps are adhered to both ends of the piezoelectric stack to create the fixation plate. These endcaps are printed on a FormLabs 2 using the FormLabs Dental LT resin (RS-F2-DLCL-01; Somerville, MA, USA). These endcaps allow the fixation plate to be attached to the femur using screws. Two stainless steel riser plates are also attached to these endcaps via screws in order to elevate the fixation plate above the site of callus formation. 
The active fixation plate described in this chapter is rated for voltages up to $75 \mathrm{~V}$. At this maximum voltage, approximately $10 \mu \mathrm{m}$ of displacement can be obtained in air, allowing this device to operate in a wide range of LMHF magnitudes (up to $10 \%$ of functional loading). Piezoelectric actuators are known for their fast response time, and actuation frequency is typically limited by the slew rate of the amplifier system instead of the material itself. LMHF frequencies of less than $100 \mathrm{~Hz}$ are easily obtainable, and the most common actuation frequency of $35 \mathrm{~Hz}$ is demonstrated. The loading sensitivity of this plate is roughly $25 \mathrm{mV} / \mathrm{N}$ when not compressing a solid material. This sensitivity decreases when compressing solid materials to roughly $24 \mathrm{mV} / \mathrm{N}$ at $12 \mathrm{MPa}$ (simulating cartilage) and to $7 \mathrm{mV} / \mathrm{N}$ at $232 \mathrm{MPa}$ (simulating the hard callus) so that the stiffness of the bone may be compared longitudinally over time. The plate is coated in parylene-C and shows no immediate decrease in either osteoblast viability or relative live/dead counts during the first few days of exposure.

\subsection{The Piezoelectric Effect}

Certain materials have properties that result in a relationship between the shape of the material and its charge distribution. The effects caused by this relationship are known as piezoelectric effects. The direct piezoelectric effect, first demonstrated in 1880 [59], describes how physical deformation of these materials result in a measurable voltage across that material. The inverse piezoelectric effect, first demonstrated in 1881 [60], describes how the application of an electric field to these materials results in physical deformation.

Because of these properties, piezoelectric materials may be used as both actuators and sensors. Piezoelectric actuators are commonly used for high precision displacements [61, 62] or MEMS applications [63]. Piezoelectric sensors are often used in MEMS devices as pressure sensors [64], microphones [65], and strain sensors [66, 67]. 


\subsubsection{Mechanics of Piezoelectric Materials}

Many piezoelectric materials, including the material used in this fixation plate, are ceramics composed of poled domains containing a perovskite crystal structure (Figure 2.2). These crystals contain a charged ion at the center of the unit cell $\left(\mathrm{Ti}^{4+}\right.$ or $\mathrm{Zr}^{4+}$ in the case of PZT). This ion moves away from the center of the unit cell when the unit cell is strained, resulting in a displacement of charge. Similarly, if the center ion is pushed away from center due to the presence of an electric field, the unit cell around it will strain to a new low energy configuration.

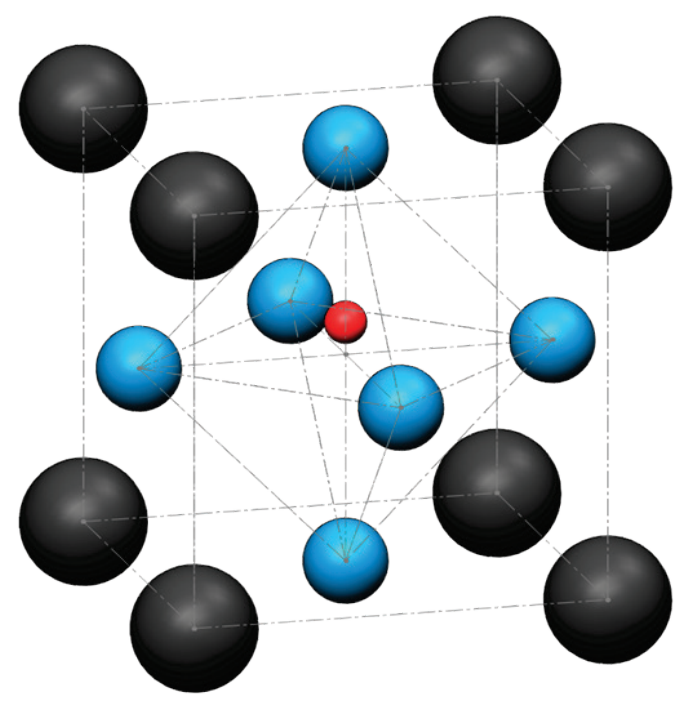

Figure 2.2 The unit cell of PZT. The black atoms (corners) represent $\mathrm{Pb}^{2+}$; the blue atoms (face centers) represent $\mathrm{O}^{2-}$; the red atom (body center) represents $\mathrm{Ti}^{4+}$ or $\mathrm{Zr}^{4+}$.

A typical piezoelectric material contains many domains containing these unit cells aligned in different directions. These domains are reoriented so that they are aligned in the same direction through a process called poling. This results in a material that can have a microscale or greater actuation performance. The behavior of a piezoelectric material is shown in Figure 2.3. 
(a)

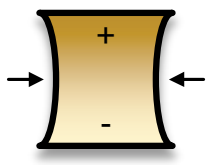

(c)

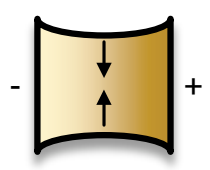

(b)

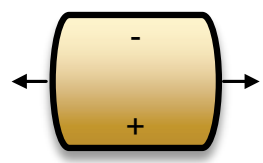

(d)

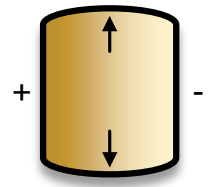

Figure 2.3 Simplified behavior of a piezoelectric material, showing that $(a, b)$ applying stress to the material results in a separation of charge and (c, d) applying a voltage field across the material results in an induced strain.

The actuation displacement (commonly referred to as the free stroke) is a function of the applied stress and voltage, not the size of the actuator. Because of this, most linear actuators are made using stacks of smaller piezoelectric elements. For example, the piezoelectric stack used in this work features a pair of interdigitated electrodes (Figure 2.4) that apply voltage to smaller portions of the piezoelectric material to increase the actuation performance. 


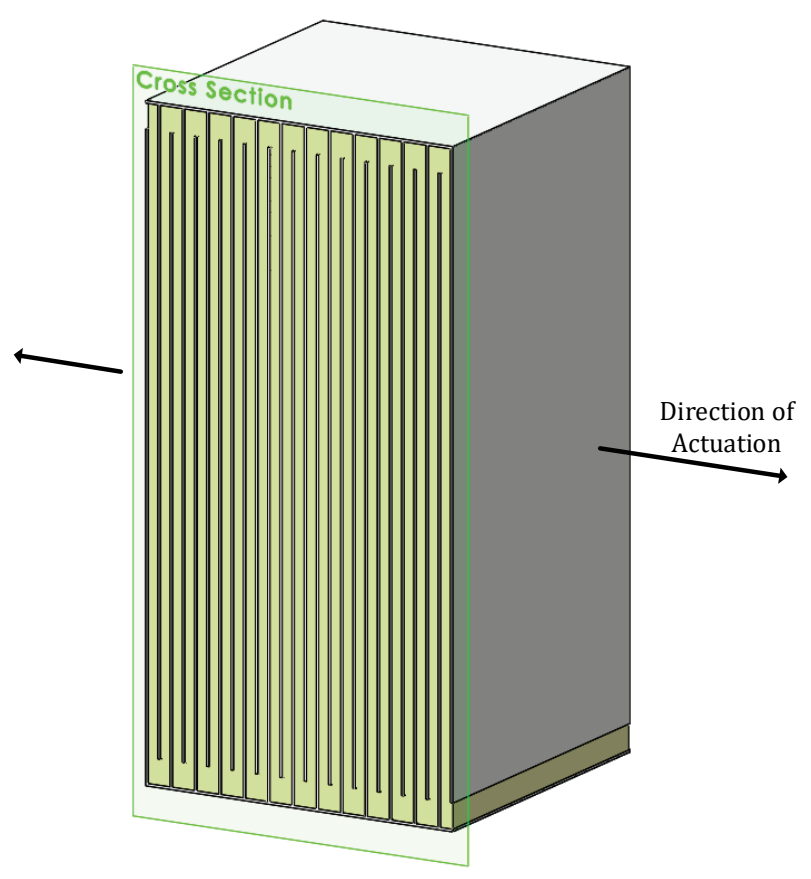

Figure 2.4 Piezoelectric actuator featuring many interdigitated electrodes to create many smaller piezoelectric actuators within a single element. The PK2FMP2 used in this work has 59 effective layers in each chip.

\subsubsection{Quantification of Piezoelectric Behavior}

The magnitude of the piezoelectric effect of a material may be specified by a constant called the piezoelectric constant. This effect may be described in terms of both physical (stress and strain) and electrical (displacement and field) parameters. Due to this, there are four different piezoelectric constants that are used to relate each physical parameter with each electrical parameter (Table 2.1) [68]. 
Table 2.1 Piezoelectric constants and their definitions [69]. $T$ is stress $\left(\mathrm{N} / \mathrm{m}^{2}\right), S$ is strain (dimensionless), $D$ is electric displacement $\left(\mathrm{C} / \mathrm{m}^{2}\right), E$ is electric field $(\mathrm{V} / \mathrm{m})$.

\begin{tabular}{|ccc|}
\hline Constant & Units & Definition \\
$d$ & $\mathrm{~m} / \mathrm{V}, \mathrm{C} / \mathrm{N}$ & $d=\frac{D}{T}=\frac{S}{E}$ \\
$g$ & $\mathrm{Vm} / \mathrm{N}, \mathrm{m}^{2} / \mathrm{V}$ & $g=\frac{E}{T}=\frac{S}{D}$ \\
$e$ & $\mathrm{C} / \mathrm{m}^{2}$ & $e=\frac{D}{S}=\frac{-T}{E}$ \\
$h$ & $\mathrm{~V} / \mathrm{m}, \mathrm{N} / \mathrm{C}$ & $h=\frac{-E}{S}=\frac{-T}{D}$ \\
\hline
\end{tabular}

Piezoelectric behaviors can be quantified using a system of two constitutive equations. There are four such systems of equations, one system for each constant described above [69]. Each equation allows for different quantities (stress, strain, electric displacement, and electric field) to be solved for. Thus the set of equations used in the description of a piezoelectric application depends on which quantities are most appropriate in that system.

In the first system of equations, the strain $S$ and electric displacement $D$ are a function of stress $T$ and electric field $E$. The constitutive equations may be written as:

$$
\begin{gathered}
S_{i j}=s_{i j k l}^{E} T_{k l}+d_{k i j} E_{k} \\
D_{i}=d_{i k l} T_{k l}+\varepsilon_{i k}^{T} E_{k}
\end{gathered}
$$

where $S$ is strain (dimensionless), $s$ is compliance $\left(\mathrm{m}^{2} / \mathrm{N}\right)$ at a constant electric field $E, T$ is stress $\left(\mathrm{N} / \mathrm{m}^{2}\right), d$ is the piezoelectric constant $(\mathrm{m} / \mathrm{V}), E$ is electric field $(\mathrm{V} / \mathrm{m}), D$ is electric displacement $\left(\mathrm{C} / \mathrm{m}^{2}\right)$, and $\varepsilon$ is permittivity $(\mathrm{F} / \mathrm{m})$.

The second system of equations represents strain $S$ and electric field $E$ as a function of stress $T$ and electric displacement $D$. The constitutive equations may be written as:

$$
S_{i j}=s_{i j k l}^{D} T_{k l}+g_{k i j} D_{k}
$$




$$
E_{i}=-g_{i k l} T_{k l}+\beta_{i k}^{S} D_{k}
$$

where $S$ is strain (dimensionless), $s$ is compliance $\left(\mathrm{m}^{2} / \mathrm{N}\right)$ at a constant electric displacement $D, T$ is stress $\left(\mathrm{N} / \mathrm{m}^{2}\right), g$ is the piezoelectric constant $(\mathrm{Vm} / \mathrm{N}), D$ is electric displacement $\left(\mathrm{C} / \mathrm{m}^{2}\right), E$ is electric field $(\mathrm{V} / \mathrm{m})$, and $\beta$ is impermittivity $(\mathrm{m} / \mathrm{F})$.

The third system of equations describes stress $T$ and electric displacement $D$ as a function of strain $S$ and electric field $E$. These equations may be written as:

$$
\begin{aligned}
& T_{i j}=c_{i j k l}^{E} S_{k l}-e_{k i j} E_{k} \\
& D_{i}=e_{i k l} S_{k l}+\varepsilon_{i j}^{S} E_{k}
\end{aligned}
$$

where $T$ is stress $\left(\mathrm{N} / \mathrm{m}^{2}\right), c$ is elastic stiffness $\left(\mathrm{N} / \mathrm{m}^{2}\right)$ at constant electric field $E, S$ is strain (dimensionless), $e$ is the piezoelectric constant $\left(\mathrm{C}^{2} \mathrm{~m}^{2}\right), E$ is electric field $(\mathrm{V} / \mathrm{m}), D$ is electric displacement $\left(\mathrm{C} / \mathrm{m}^{2}\right)$, and $\varepsilon$ is permittivity $(\mathrm{F} / \mathrm{m})$.

The final system of equations describes stress $T$ and electric field $E$ in terms of strain $S$ and electric displacement $D$. The constitutive equations may be written as:

$$
\begin{aligned}
& T_{i j}=c_{i j k l}^{D} S_{k l}-h_{k i j} D_{k} \\
& E_{i}=-h_{i k l} S_{k l}+\beta_{i k}^{S} D_{k}
\end{aligned}
$$

where $T$ is stress $\left(\mathrm{N} / \mathrm{m}^{2}\right), c$ is stiffness $\left(\mathrm{N} / \mathrm{m}^{2}\right)$ at a constant electric displacement $D, S$ is strain (dimensionless), $h$ is the piezoelectric constant $(\mathrm{V} / \mathrm{m}), D$ is the electric displacement $\left(\mathrm{C} / \mathrm{m}^{2}\right), E$ is electric field $(\mathrm{V} / \mathrm{m})$, and $\beta$ is impermittivity $(\mathrm{m} / \mathrm{F})$.

Any of these sets of equations may be used depending on which is most convenient for the application. In this work, it is convenient to work in terms of strain in order to quantify actuation performance. It is also convenient to work in terms of voltage (electric field multiplied by distance) for microcontroller-based applications. Therefore, the set of 
equations 2.3 and 2.4 will be used in this work. This system of equations may be rewritten in matrix form:

$$
\left[\begin{array}{c}
S_{i j} \\
D_{i}
\end{array}\right]=\left[\begin{array}{cc}
S_{i j k l}^{E} & d_{k i j} \\
d_{i k l} & \varepsilon_{i k}^{T}
\end{array}\right]\left[\begin{array}{c}
T_{k l} \\
E_{k}
\end{array}\right]
$$

where subscripts $i$ and $j$ represent axial and shear components of the dependent quantities, and subscripts $k$ and $l$ represent axial and shear components of the independent quantities. Piezoelectric materials are anisotropic by nature. Therefore, it is appropriate to use tensors to calculate the behavior of piezoelectric materials. For materials with a cubic crystal structure, such as PZT, the crystallographic directions 1-6 are commonly used, where 1-3 represent axial directions and 4-6 represent shear directions. These directions with reference to the piezoelectric stack used in this work are shown in Figure 2.5.

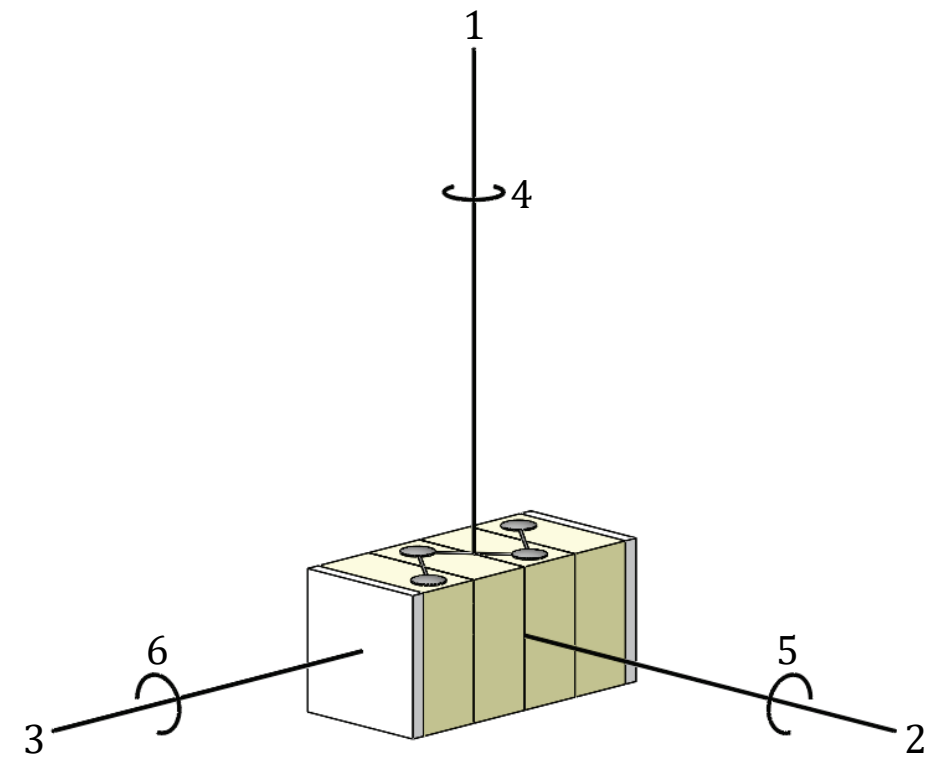

Figure 2.5 Crystallographic directions used for describing piezoelectric axes. Along the $\mathrm{Z}$-axis is direction 1 , along the $\mathrm{Y}$-axis is direction 2, along the $\mathrm{X}$-axis is direction 3 , around the $\mathrm{Z}$-axis is direction 4 , around the $\mathrm{Y}$-axis is direction 5 , and around the $\mathrm{X}$-axis is direction 6 .

Using these orientations, the components of the matrix in equation 2.9 may be represented in tensor form. The strain $S_{i j}$ is represented as axial strains $S_{l-3}$ and shear strains $S_{4-6}$ as: 


$$
S_{i j}=\left[\begin{array}{l}
S_{1} \\
S_{2} \\
S_{3} \\
S_{4} \\
S_{5} \\
S_{6}
\end{array}\right]
$$

In this work, the axial strain experienced along the fixation plate during actuation is represented by $S_{3}$.

The stress $T_{k l}$ is represented in terms of axial stress $T_{1-3}$ and shear stress $T_{4-6}$ as:

$$
T_{k l}=\left[\begin{array}{l}
T_{1} \\
T_{2} \\
T_{3} \\
T_{4} \\
T_{5} \\
T_{6}
\end{array}\right]
$$

In this work, $T_{3}$ contains the main component of the stress experienced by the fixation plate during load monitoring.

The electric displacement $D_{i}$ and electric field $E_{k}$ are described in terms of axial components only as:

$$
\begin{aligned}
D_{i} & =\left[\begin{array}{l}
D_{1} \\
D_{2} \\
D_{3}
\end{array}\right] \\
E_{k} & =\left[\begin{array}{l}
E_{1} \\
E_{2} \\
E_{3}
\end{array}\right]
\end{aligned}
$$

During actuation, the electric field is applied as $E_{3}$. During load monitoring, $D_{3}$ contains the electric displacement that will be measured by the device.

The physical compliance of the material $s_{i j k l}$ at constant electric field $E$ is represented in terms of axial and shear directions as: 


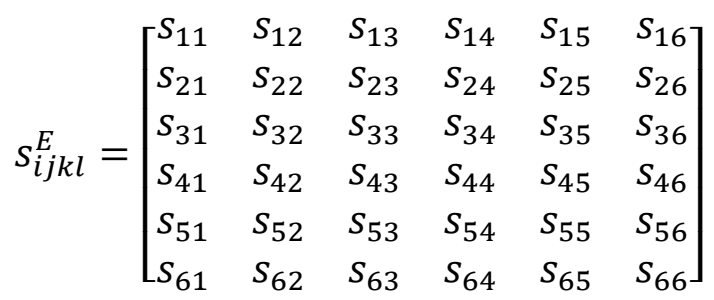

so that each component of the matrix $s_{i j k l}$ describes the displacement caused in the $i j$ direction caused by a force in the $k l$ direction. For example, $s_{12}$ describes how a material deforms in the 1 direction when loaded in the 2 direction. Due to the limited shear compliance of most piezoelectric materials [69, 70], equation 2.14 may usually be simplified to:

$$
s_{i j k l}^{E}=\left[\begin{array}{cccccc}
s_{11} & s_{12} & s_{13} & 0 & 0 & 0 \\
s_{21} & s_{22} & s_{23} & 0 & 0 & 0 \\
s_{31} & s_{32} & s_{33} & 0 & 0 & 0 \\
0 & 0 & 0 & s_{44} & 0 & 0 \\
0 & 0 & 0 & 0 & s_{55} & 0 \\
0 & 0 & 0 & 0 & 0 & s_{66}
\end{array}\right]
$$

The piezoelectric constants $d_{i k l}$ and $d_{k i j}$ representing the relationship between voltage and displacement are represented by:

$$
\begin{aligned}
d_{i k l} & =\left[\begin{array}{llllll}
d_{11} & d_{12} & d_{13} & d_{14} & d_{15} & d_{16} \\
d_{21} & d_{22} & d_{23} & d_{24} & d_{25} & d_{26} \\
d_{31} & d_{32} & d_{33} & d_{34} & d_{35} & d_{36}
\end{array}\right] \\
d_{k i j} & =\left[\begin{array}{lll}
d_{11} & d_{21} & d_{31} \\
d_{12} & d_{22} & d_{32} \\
d_{13} & d_{23} & d_{33} \\
d_{14} & d_{24} & d_{34} \\
d_{15} & d_{25} & d_{35} \\
d_{16} & d_{26} & d_{36}
\end{array}\right]
\end{aligned}
$$

so that $d_{i k l}$ describes how the electric field in the $k l$ direction influence strain in the $i$ direction, and so that $d_{k i j}$ describes how the strain in the $i j$ direction influence the electric field in the $k$ direction. In a piezoelectric material polled in the 3 direction, these can usually simplify as [70]: 


$$
\begin{aligned}
d_{i k l} & =\left[\begin{array}{cccccc}
d_{11} & d_{12} & d_{13} & 0 & 0 & 0 \\
0 & 0 & 0 & d_{24} & 0 & 0 \\
0 & 0 & 0 & 0 & d_{35} & 0
\end{array}\right] \\
d_{k i j} & =\left[\begin{array}{ccc}
0 & 0 & d_{31} \\
0 & 0 & d_{32} \\
0 & 0 & d_{33} \\
0 & d_{24} & 0 \\
d_{15} & 0 & 0 \\
0 & 0 & 0
\end{array}\right]
\end{aligned}
$$

Finally, the permittivity of the material $\varepsilon_{i k}$ at a constant stress $T$ may be described as:

$$
\varepsilon_{i k}^{T}=\left[\begin{array}{lll}
\varepsilon_{11} & \varepsilon_{12} & \varepsilon_{13} \\
\varepsilon_{21} & \varepsilon_{22} & \varepsilon_{23} \\
\varepsilon_{31} & \varepsilon_{32} & \varepsilon_{33}
\end{array}\right]
$$

and may generally be reduced to:

$$
\varepsilon_{i k}^{T}=\left[\begin{array}{ccc}
\varepsilon_{11} & 0 & 0 \\
0 & \varepsilon_{22} & 0 \\
0 & 0 & \varepsilon_{33}
\end{array}\right]
$$

Substituting equations $10-14,16,17$, and 20 into equation 9 yields:

$$
\left[\begin{array}{l}
S_{1} \\
S_{2} \\
S_{3} \\
S_{4} \\
S_{5} \\
S_{6} \\
D_{1} \\
D_{2} \\
D_{3}
\end{array}\right]=\left[\begin{array}{lllllllll}
s_{11} & s_{12} & s_{13} & s_{14} & s_{15} & s_{16} & d_{11} & d_{21} & d_{31} \\
s_{21} & s_{22} & s_{23} & s_{24} & s_{25} & s_{26} & d_{12} & d_{22} & d_{32} \\
s_{31} & s_{32} & s_{33} & s_{34} & s_{35} & s_{36} & d_{13} & d_{23} & d_{33} \\
s_{41} & s_{42} & s_{43} & s_{44} & s_{45} & s_{46} & d_{14} & d_{24} & d_{34} \\
s_{51} & s_{52} & s_{53} & s_{54} & s_{55} & s_{56} & d_{15} & d_{25} & d_{35} \\
s_{61} & s_{62} & s_{63} & s_{64} & s_{65} & s_{66} & d_{16} & d_{26} & d_{36} \\
d_{11} & d_{12} & d_{13} & d_{14} & d_{15} & d_{16} & \varepsilon_{11} & \varepsilon_{12} & \varepsilon_{13} \\
d_{21} & d_{22} & d_{23} & d_{24} & d_{25} & d_{26} & \varepsilon_{21} & \varepsilon_{22} & \varepsilon_{23} \\
d_{31} & d_{32} & d_{33} & d_{34} & d_{35} & d_{36} & \varepsilon_{31} & \varepsilon_{32} & \varepsilon_{33}
\end{array}\right]\left[\begin{array}{l}
T_{1} \\
T_{2} \\
T_{3} \\
T_{4} \\
T_{5} \\
T_{6} \\
E_{1} \\
E_{2} \\
E_{3}
\end{array}\right]
$$

or, when using the simplified equations $15,18,19$, and 21 yields: 


$$
\left[\begin{array}{l}
S_{1} \\
S_{2} \\
S_{3} \\
S_{4} \\
S_{5} \\
S_{6} \\
D_{1} \\
D_{2} \\
D_{3}
\end{array}\right]=\left[\begin{array}{ccccccccc}
S_{11} & S_{12} & s_{13} & 0 & 0 & 0 & 0 & 0 & d_{31} \\
S_{21} & S_{22} & S_{23} & 0 & 0 & 0 & 0 & 0 & d_{32} \\
S_{31} & S_{32} & S_{33} & 0 & 0 & 0 & 0 & 0 & d_{33} \\
0 & 0 & 0 & s_{44} & 0 & 0 & 0 & d_{24} & 0 \\
0 & 0 & 0 & 0 & S_{55} & 0 & d_{15} & 0 & 0 \\
0 & 0 & 0 & 0 & 0 & s_{66} & 0 & 0 & 0 \\
0 & 0 & 0 & 0 & d_{15} & 0 & \varepsilon_{11} & 0 & 0 \\
0 & 0 & 0 & d_{24} & 0 & 0 & 0 & \varepsilon_{22} & 0 \\
d_{31} & d_{32} & d_{33} & 0 & 0 & 0 & 0 & 0 & \varepsilon_{33}
\end{array}\right]\left[\begin{array}{c}
T_{1} \\
T_{2} \\
T_{3} \\
T_{4} \\
T_{5} \\
T_{6} \\
E_{1} \\
E_{2} \\
E_{3}
\end{array}\right]
$$

To solve for the strain induced in the direction of actuation $S_{3}$, Equation 2.23 simplifies to:

$$
S_{3}=s_{31} T_{1}+s_{32} T_{2}+s_{33} T_{3}+d_{33} E_{3}
$$

or, when $T_{l-3}=0$ this may be written in terms of displacement $\Delta X$ and voltage $\Delta V$ in place of strain $S$ and voltage field $E$ :

$$
\Delta X_{3}=d_{33} \Delta V_{3}
$$

According to the manufacturer, the sensitivity of piezoelectric actuation $d_{33}$ for this material is $0.710 \mathrm{~nm} / \mathrm{V}$ [71]. However, this is the sensitivity of each piezoelectric element, not the entire stack. The stack contains 4 chips, each consisting of 59 effective layers. Therefore, the sensitivity of the stack as a whole is expected to be $167 \mathrm{~nm} / \mathrm{V}$. Using Equation 2.25, the expected displacement of the actuator at $75 \mathrm{~V}$ when no load is applied to the fixation plate $\left(T_{1-3}=0\right)$ is $12.6 \mu \mathrm{m}$ (cf. advertised experimental displacement of 11.2 $\mu \mathrm{m})$.

A similar derivation may be done using Equations 2.3 and 2.4 to identify the electric field $E_{i}$ of the actuator as a function of applied load $T_{k l}$. The result of this derivation for the electric field in the direction of actuation alone $E_{3}$ in terms of stress $T$ and electric displacement $D$ is:

$$
E_{3}=g_{31} T_{1}+g_{32} T_{2}+g_{33} T_{3}+\beta_{33} D_{3}
$$


Where $g_{31-33}$ represents the piezoelectric constant in terms of $\mathrm{V} / \mathrm{N}$, and $\beta$ represents the impermittivity of the material in terms of $\mathrm{m} / \mathrm{F}$. The sensitivity $g_{33}$ provided by the manufacturer is $25 \mathrm{mV} / \mathrm{N}$ [71]. This normalized version of the $g_{33}$ constant represents the expected loading sensitivity of the actuator.

\subsubsection{Nonlinear Behaviors}

Real piezoelectric materials exhibit several types of nonlinear behaviors. These behaviors - which include creep, hysteresis, and drift—should be taken into consideration when using piezoelectric materials as sensors and actuators.

Creep during actuation is a major concern in applications which use piezoelectric devices for precision micropositioning [72]. When voltage is applied to a piezoelectric actuator, it is common to observe damping of the target displacement. Figure 2.6 shows the settling behavior of the bone plate in use when $15 \mathrm{~V}$ is applied. Creep is not likely to be a significant concern in this application because the precision required during actuation is not as precise as what is needed in other applications like microscopy.

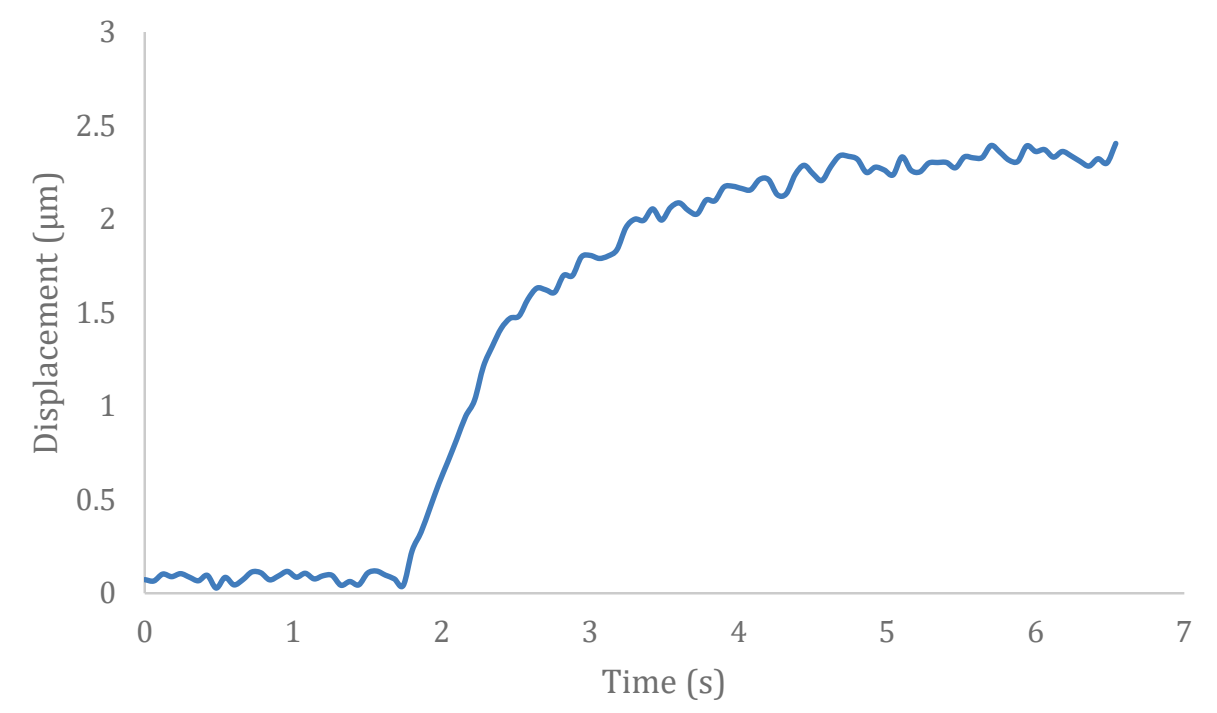

Figure 2.6 Sample of creep behavior during the application of $15 \mathrm{~V}$ step to the actuator. 
Hysteresis is present in piezoelectric materials under both actuation and loading [73]. Defects in the material such as vacancies and domain walls inhibit uniform reorientation of charge in the material [74]. The piezoelectric constants have been often described as complex numbers that account for this phase behavior [75]. This adds complexity to the use of piezoelectric materials as linear actuators and sensors because this hysteresis must be accounted for. However, when measuring the magnitude of cyclic loading, the hysteresis is less important. This is true for both cyclic actuation and load monitoring. An example of the hysteresis present in this device is shown in Figure 2.7. Piezoelectric materials such as the one used in this device typically show much more significant hysteresis, but this may be damped by the compliant and off-axis nature of the fixation plate design.

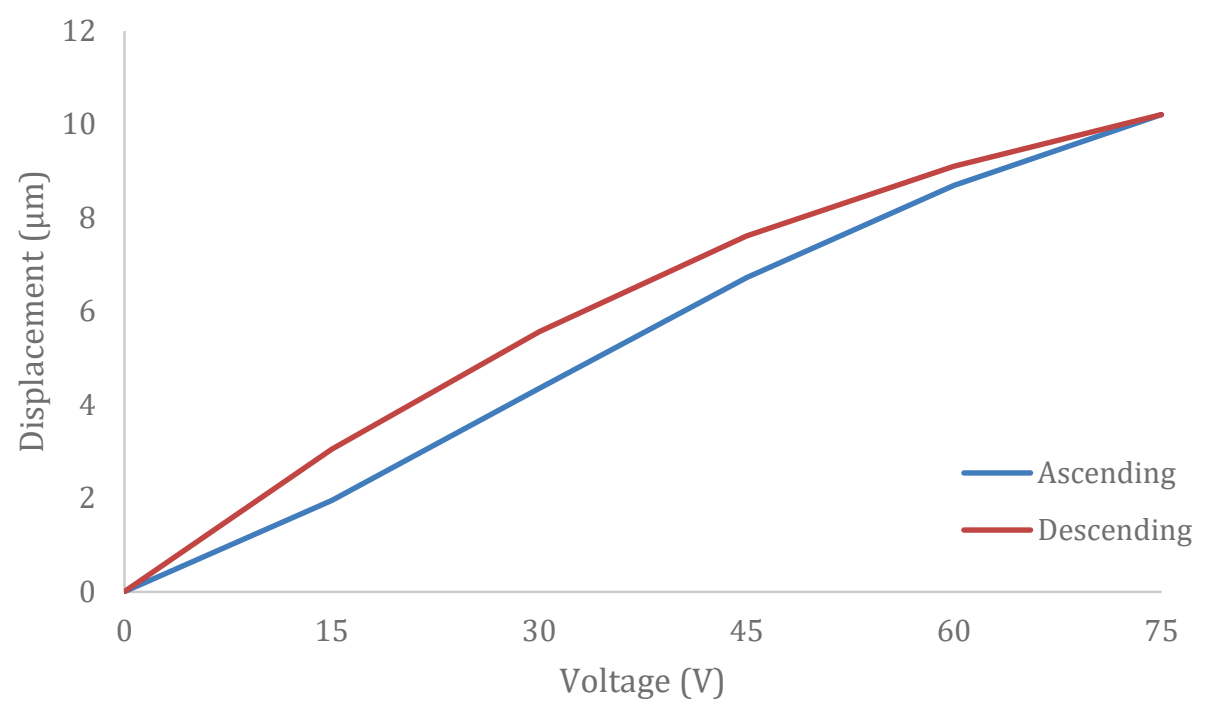

Figure 2.7 Sample of hysteresis present under cyclic actuation. The maximum hysteresis in this sample is approximately $10 \%$ of the maximum displacement.

Drift can be a significant issue in some sensing applications using piezoelectric materials. However, drift is less significant as long as the magnitude of the load being measured is relatively high and the measurement duration is relatively short [76]. Differential measurements can be used for measuring the cyclic loading that occurs during ambulation. Figure 2.8 shows an example of significant voltage drift occurring while measuring the application of a triangular cyclic load. The amplitude of the signal (the volume of interest 
in this application) may be clearly extracted, and post-processing may be used to account for the drift over time intervals on the order of minutes.

(a)

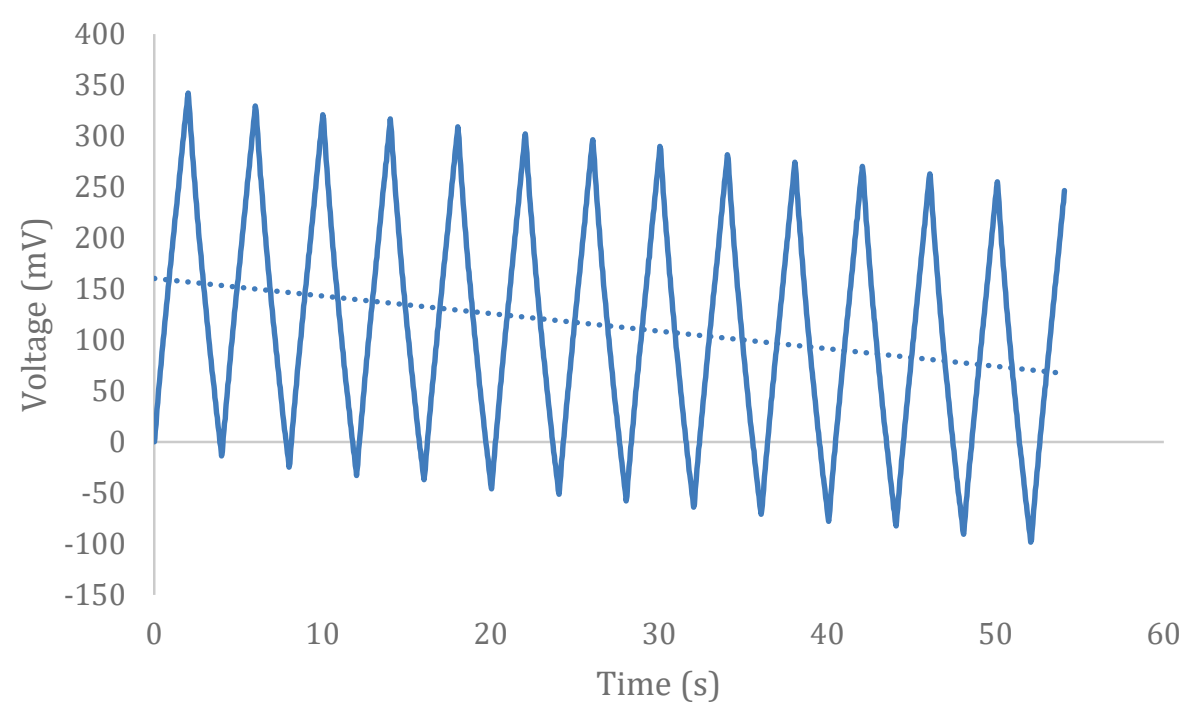

(b)

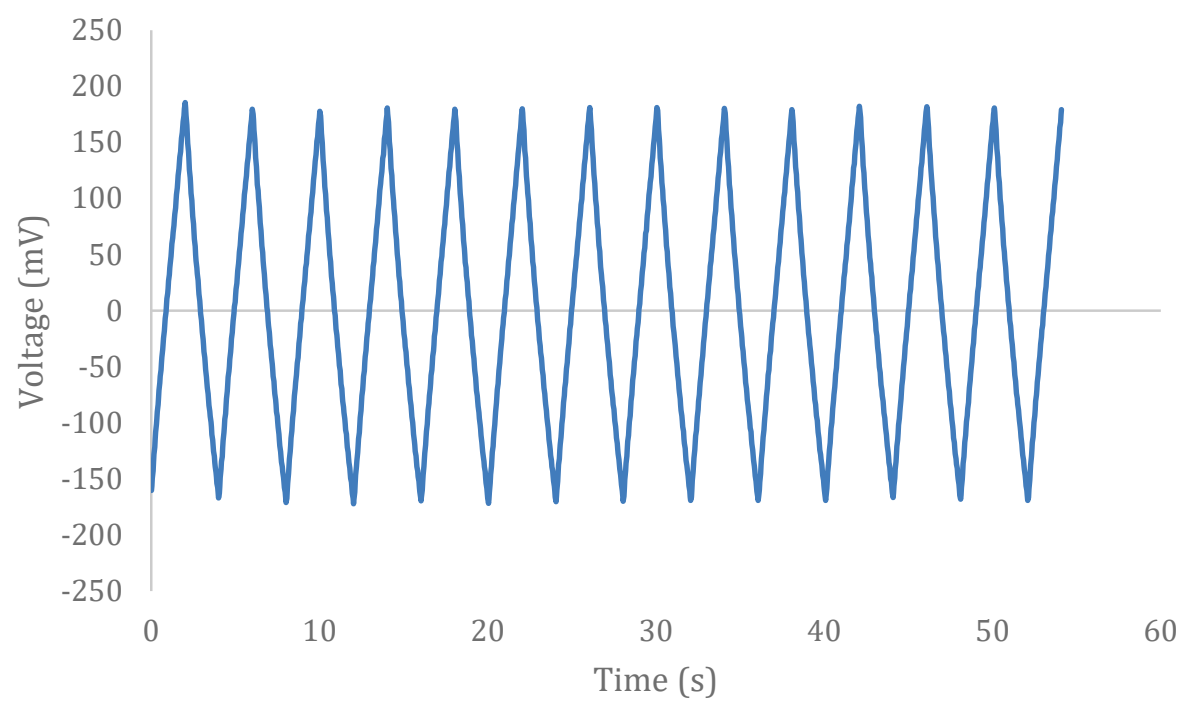

Figure 2.8 (a) Example of sensor drift during triangular loading in one of the more extreme cases. The average drift in this sample is $-1.7 \mathrm{mV} / \mathrm{s}$. The post-processed sample is shown in (b). 
While drift may bring the voltage measurements out of range of a particular ADC, this may be accounted for by the application of $0 \mathrm{~V}$ across the material before measurement. The drift tends toward $0 \mathrm{~V}$ in the absence of external loading. Drift in the load monitoring data may be accounted for using computer post-processing.

\subsection{Fabrication}

The endcaps for the fixation plate are 3D printed using the FormLabs Dental LT resin (RSF2-DLCL-01) using recommended washing and curing cycles. Before curing, the endcaps are sanded and the holes are drilled and countersunk to maintain greater uniformity. These are attached to the ceramic ends of the piezoelectric stack using TS10 Torr Seal (ThorLabs, Newton, NJ, USA) and cured for 2 hours at $60^{\circ} \mathrm{C}$.

Once the plates are fabricated, the film around the piezoelectric stack is removed, and the wires provided by the manufacturer are unsoldered. Two PFA-coated stainless-steel wires (A-M Systems, Sequim, WA, USA) are cut to 5 inches in length and glued together. These wires are inserted into a silicone sleeve (A-M Systems, Sequim, WA, USA) with diameter 0.025 inches. Omnetics nano-contacts (Omnetics Connector Corporation, Minneapolis, $\mathrm{MN}, \mathrm{USA}$ ) are crimped to the exposed ends of each wire and soldered to the piezoelectric stack. A $25 \mu \mathrm{m}$ layer of parylene-C was applied to the finished plates using the SCS Labcoter 2 (Specialty Coating Systems, Indianapolis, IN, USA). Two 316L stainless steel riser plates $(0.25 \times 0.20 \times 0.075$ inch $)$ are attached to the endcaps of the actuator using screws.

\subsection{Performance}

The actuation performance of the fixation plate was measured using the Electroforce 3200 mechanical tester (TA Instruments, New Castle, DE, USA) mechanical tester. The fixation plate was attached to two 6061 aluminum blocks with a gap between them to simulate a fractured bone following previously established methods [29]. Rubber (Young's modulus $=12 \mathrm{MPa}$ ) and Teflon (Young's modulus =232 MPa) testing blocks were inserted into the 
gap to simulate the stiffness of the soft callus and immature bone (Young's modulus $=10$ $\mathrm{MPa}$ and $1000 \mathrm{MPa}$ [36]) respectively. The testing configurations are shown in Figure 2.9.
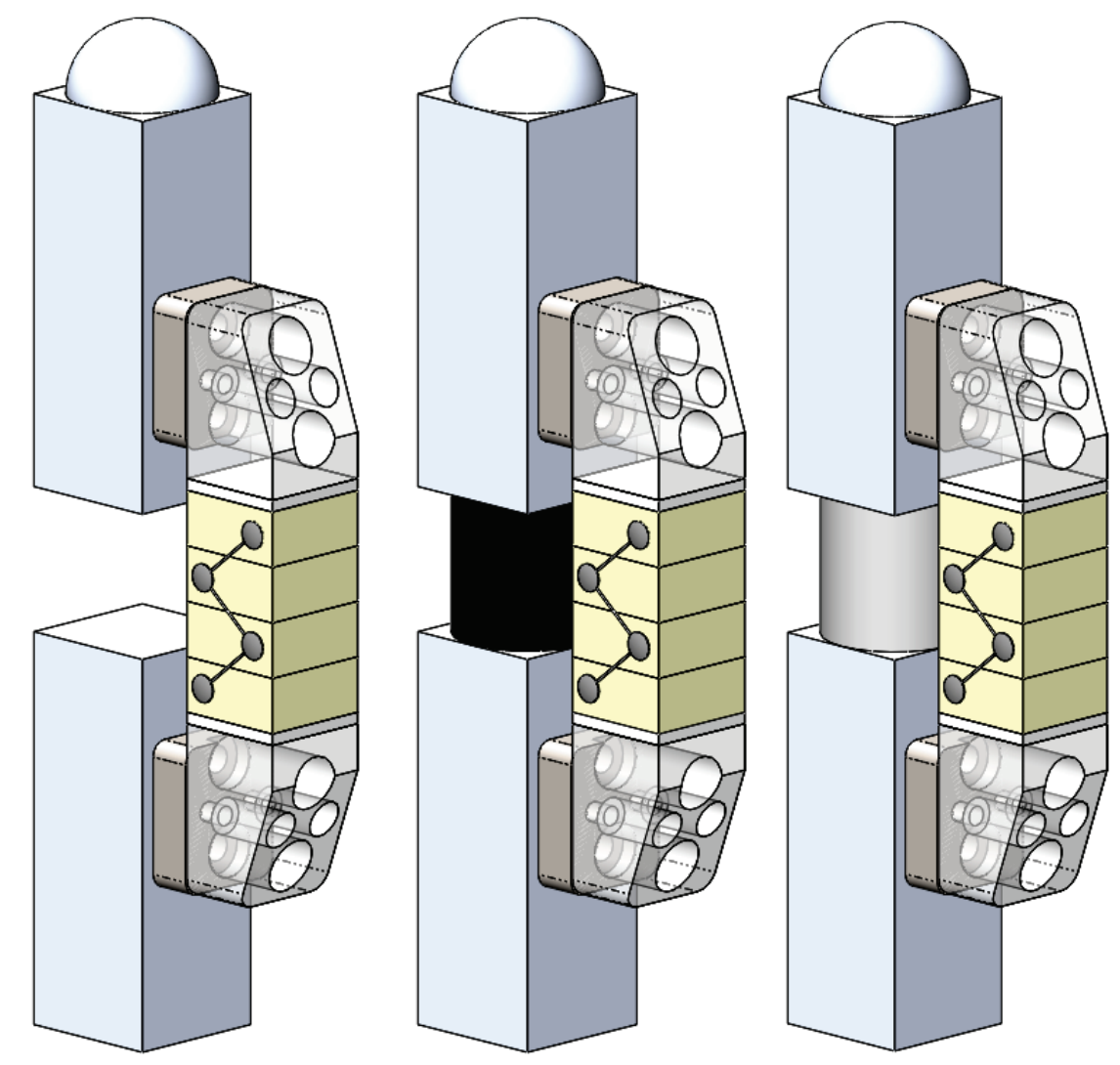

Figure 2.9 Mechanical testing configuration. Left: air gap to simulate an unbridged fracture. Center: rubber gap (stiffness $=12 \mathrm{MPa}$ ) to simulate cartilaginous callus. Right: Teflon gap (stiffness $=232 \mathrm{MPa}$ ) to simulate immature cortical bone.

\subsubsection{Actuation}

Constant blocking forces of 2, 5, and $10 \mathrm{~N}$ were applied to the fixation plate by the mechanical tester. Voltages from 0 to $75 \mathrm{~V}$ were applied to the fixation plate bidirectionally in $15 \mathrm{~V}$ increments and the resulting displacement was measured. The results are shown in Figure 2.10. 
(a)

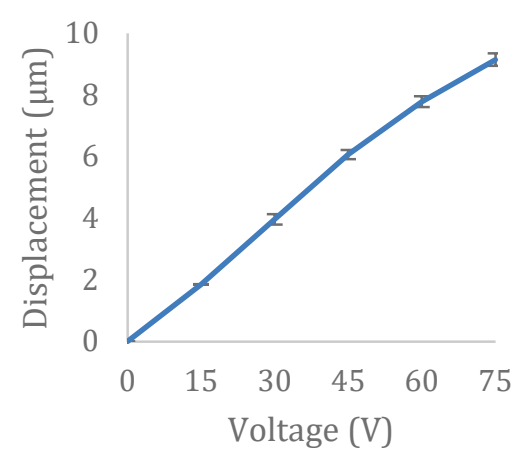

(b)

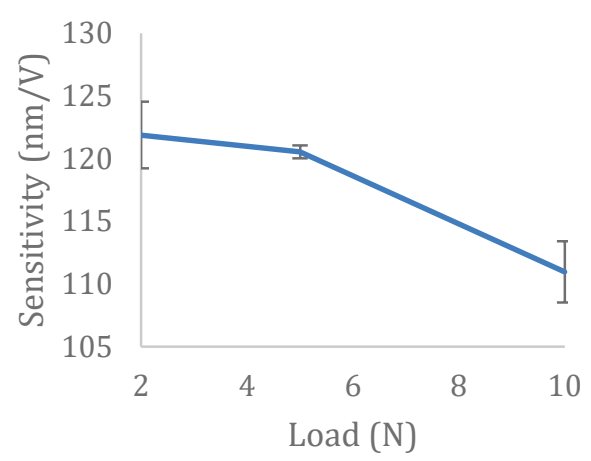

(c)

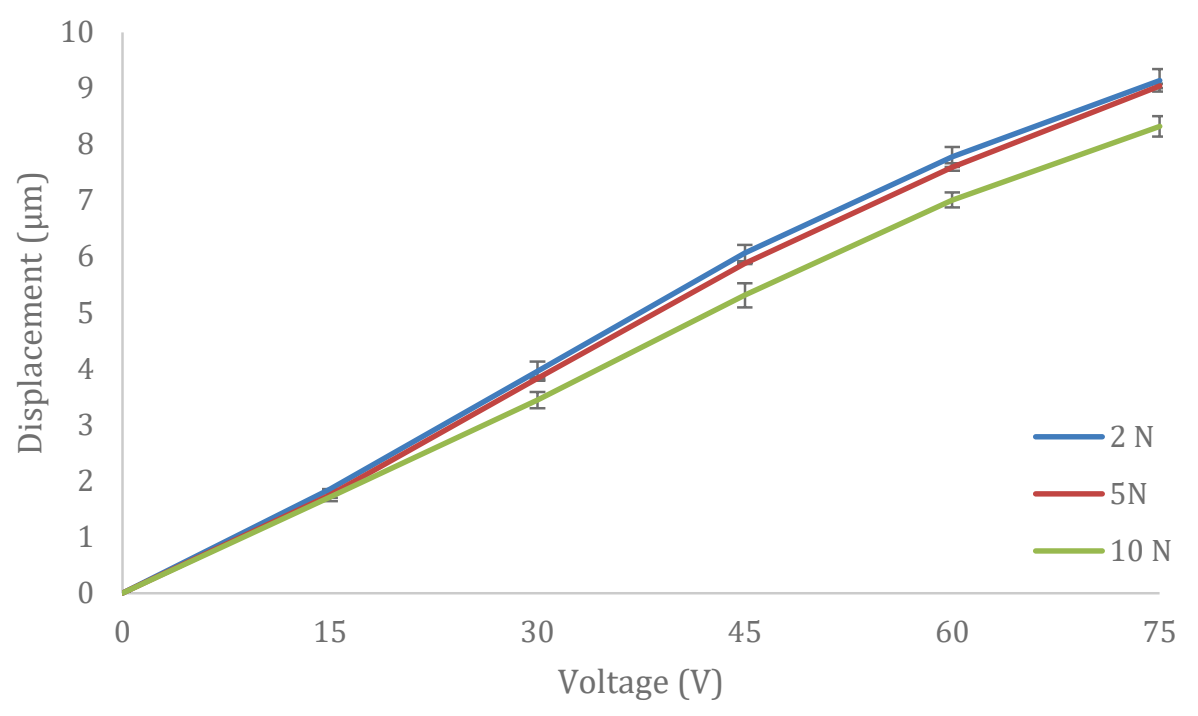

Figure 2.10 Actuation performance of the fixation plate (a) measured at a constant blocking force of $2 \mathrm{~N}$, (b) under different blocking forces from 2-10 N, and (c) showing how the actuation performance changes with blocking force.

The displacement provided by the fixator is roughly linear with the applied voltage. There is a decrease in sensitivity as the fixator approaches its rated voltage. The actuation sensitivity of the plate under low blocking force $(2 \mathrm{~N})$ is $126.4 \mathrm{~nm} / \mathrm{V}(\sigma=8.4 \mathrm{~nm} / \mathrm{V}, n=$ $3)$. Under higher blocking force $(10 \mathrm{~N})$, this decreases to $111.0 \mathrm{~nm} / \mathrm{V}(n=1)$.

The blocking force, or load resisting piezoelectric actuation, influences the sensitivity of the piezoelectric actuator. The difference in actuation sensitivity during the course of bone healing was investigated by changing the material in the gap between the aluminum testing 
blocks. An air gap was used to simulate a fracture without bridging. A rubber insert was used to simulate the presence of the soft callus after bridging. A Teflon insert was used to simulate the stiffness of immature bone. The sensitivities of the actuator when compressing these materials is shown in Figure 2.11. A one-way ANOVA shows that the material has a clearly significant effect on the actuation sensitivity of the fixator $(F(2,6)=4123.224, p<$ $0.001)$.

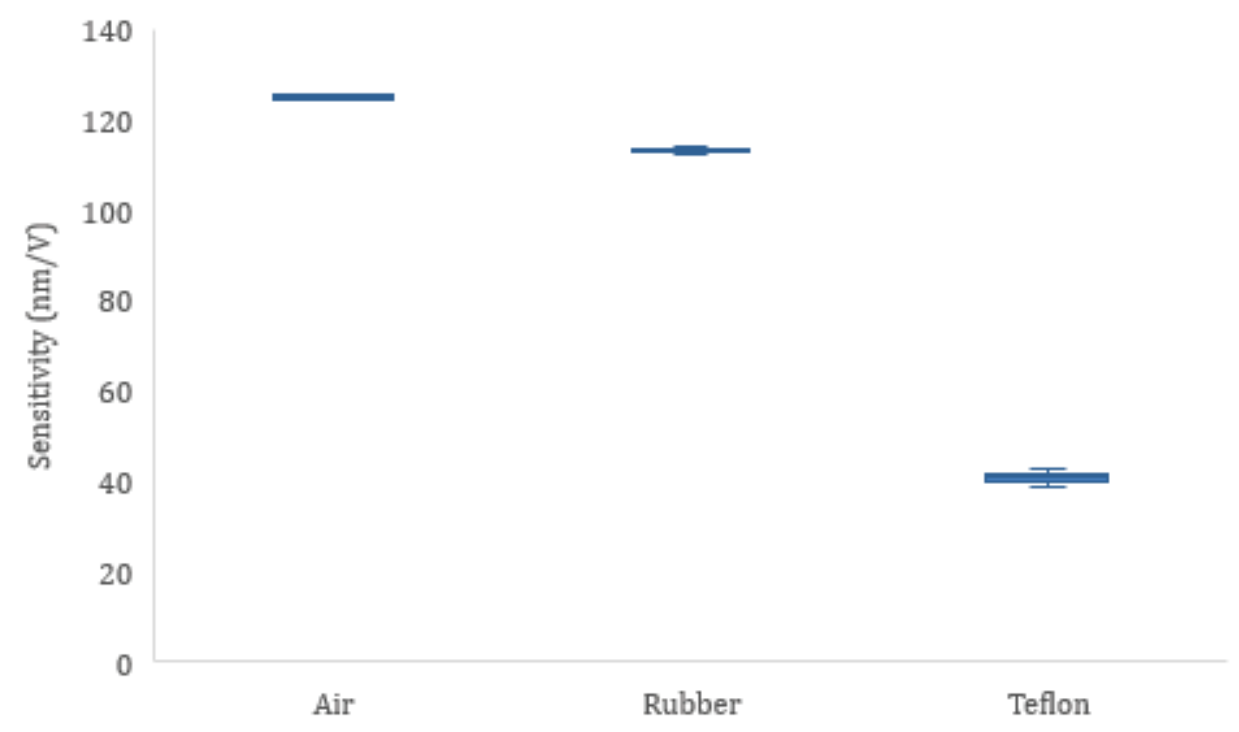

Figure 2.11 Actuation sensitivity with air, rubber, and Teflon gaps. The sensitivity in air is $124.9 \mathrm{~nm} / \mathrm{V}(\sigma=0.8 \mathrm{~nm} / \mathrm{V}, n=3)$, the sensitivity in rubber is $113.1 \mathrm{~nm} / \mathrm{V}$ $(\sigma=1.0 \mathrm{~nm} / \mathrm{V}, n=3)$, the sensitivity in Teflon is $40.8 \mathrm{~nm} / \mathrm{V}(\sigma=1.8 \mathrm{~nm} / \mathrm{V}, n=3)$.

A sample of the actuation performance of the fixation plate on the air gap is shown in Figure 2.12. The frequency of actuation is $31.25 \mathrm{~Hz}$, and the amplitude is approximately $0.7 \mu \mathrm{m}$. These match the desired profile of LMHF actuation in this animal model. The peaks of the sinusoid show significant distortion. These artifacts may be due to vibration against the mechanical tester, due to the mechanical tester attempting to maintain a constant load, or they may be present in the actuation profile itself. Even if these artifacts are present, the profile closely represents the desired LMHF regime. 


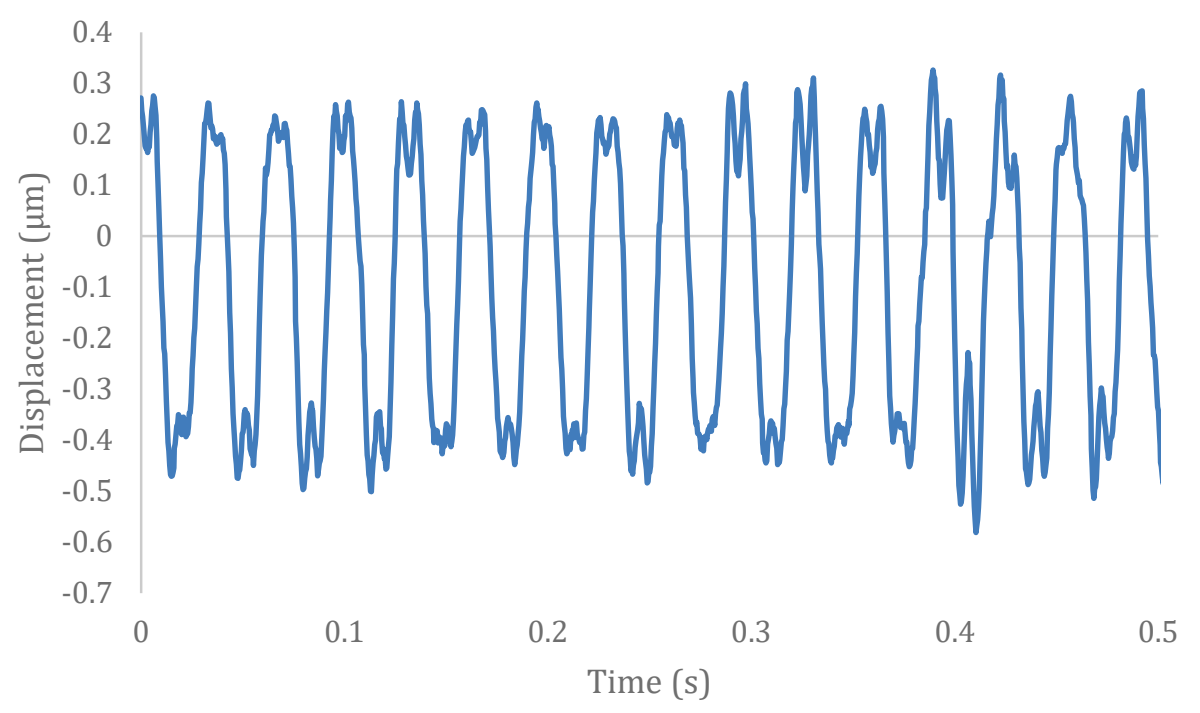

Figure 2.12 Sample of the actuation profile of the fixation plate (amplitude $=0.7$ $\mu \mathrm{m}$, frequency $=31.25 \mathrm{~Hz}$ ).

\subsubsection{Load Monitoring}

The load monitoring properties of the piezoelectric fixator show highly linear behaviors. The sensitivity of the fixation plate was measured by applying cyclic triangular waveforms to the testing configuration with an air gap. The results (Figure 2.13) show that the sensitivity of load monitoring is approximately $26.5 \mathrm{mV} / \mathrm{N}$. There are deviations in this sensitivity between fixators due to manufacturing errors resulting in sensitivities from 21.3 $\mathrm{mV} / \mathrm{N}$ to $26.8 \mathrm{mV} / \mathrm{N}$. 
(a)

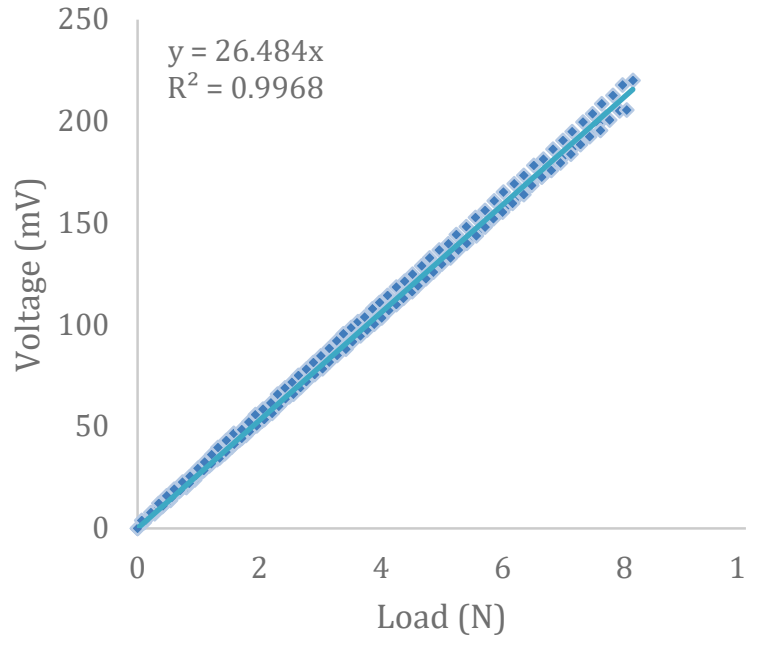

(b)

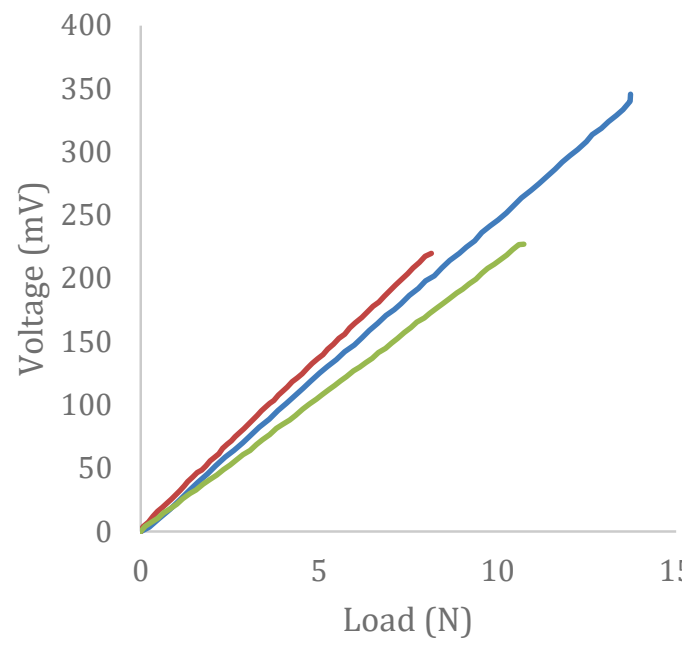

Figure 2.13 Sensitivity to loading of the fixation plate without any gap material. (a) Intraplate sensitivity $(n=3)$ of $26.3 \mathrm{mV} / \mathrm{N}$; and (b) interplate sensitivity $(n=3)$ where the sensitivity ranges from $21.3 \mathrm{mV} / \mathrm{N}$ to $26.8 \mathrm{mV} / \mathrm{N}$.

Rubber and Teflon testing blocks were inserted between the aluminum blocks to simulate the stiffness of the soft callus and immature bone to observe how the sensitivity of the fixator changes throughout the fracture healing process. Figure 2.14 shows how the sensitivity decreases from $26 \mathrm{mV} / \mathrm{N}$ in air to $7 \mathrm{mV} / \mathrm{N}$ in Teflon. A one-way ANOVA shows a significant difference between the sensitivities of the three materials $(F(2,27)=2692.384$, $p<0.001)$. 


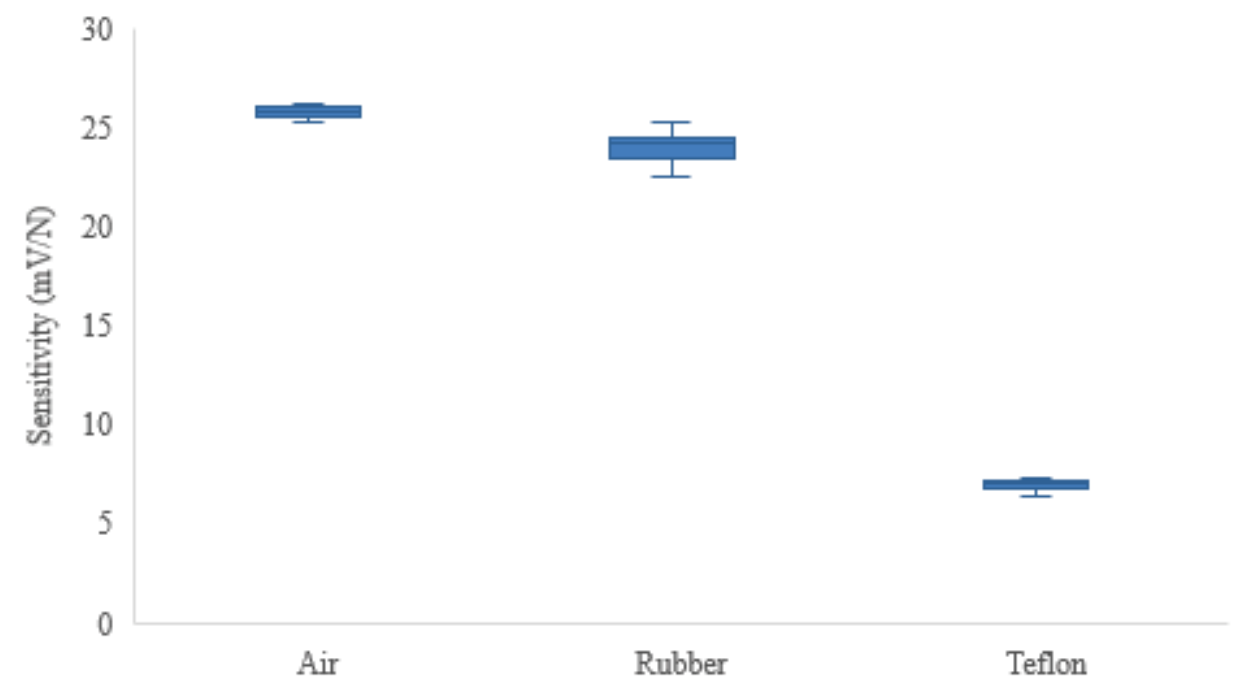

Figure 2.14 Loading sensitivity in different materials. The sensitivity in air is 25.75 $\mathrm{mV} / \mathrm{N}(\sigma=0.33 \mathrm{mV} / \mathrm{N}, n=3)$, the sensitivity in rubber is $23.96 \mathrm{mV} / \mathrm{N}(\sigma=0.87$ $\mathrm{mV} / \mathrm{N}, n=3)$, and the sensitivity in Teflon is $7.08 \mathrm{mV} / \mathrm{N}(\sigma=0.56 \mathrm{mV} / \mathrm{N}, n=3)$.

Figure 2.15 shows the voltage output of the fixation plate under cyclic loading with an air gap similar to what is experienced in vivo. Under a $0.1 \mathrm{~mm}$ cyclic load, the average voltage difference is approximately $339 \mathrm{mV}$.

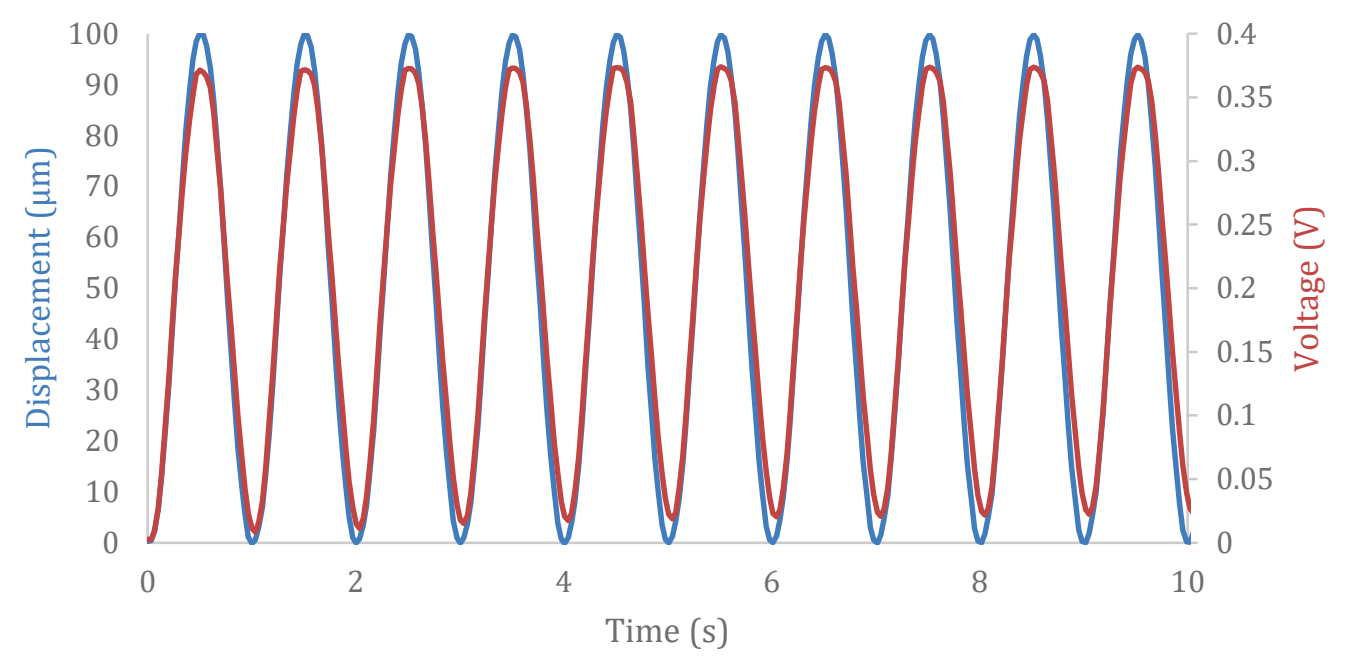

Figure 2.15 Voltage output of the fixation plate under sinusoidal compressive loading. The voltage difference measured when applying a $100 \mu \mathrm{m}$ load corresponds to an average of $339 \mathrm{mV}(\sigma=14 \mathrm{mV}, n=1)$. 


\subsubsection{Fatigue Testing}

Fatigue testing was performed on the fixation plates $(n=1)$ for 10,000 cycles to simulate physiological loading (displacement $=0.1 \mathrm{~mm}$, frequency $=1 \mathrm{~Hz}$ ). A triangular displacement of $0.1 \mathrm{~mm}$ was applied to the fixation plate both before and after the fatigue testing so that the sensing performance could be compared.

None of the plates tested broke during fatigue testing. Prior to the fatigue test (Figure $2.16 \mathrm{a})$, the fixation plate had a sensitivity of $27.406 \mathrm{mV} / \mathrm{N}(\sigma=0.483 \mathrm{mV} / \mathrm{N})$. After the fatigue test (Figure 2.16b), the plate had a sensitivity of $27.569 \mathrm{mV} / \mathrm{N}(\sigma=0.680 \mathrm{mV} / \mathrm{N})$. This shows that the loading sensitivity of the fixator does not change significantly over the lifetime of the device $(t=-0.685, p=0.501)$. 
(a)

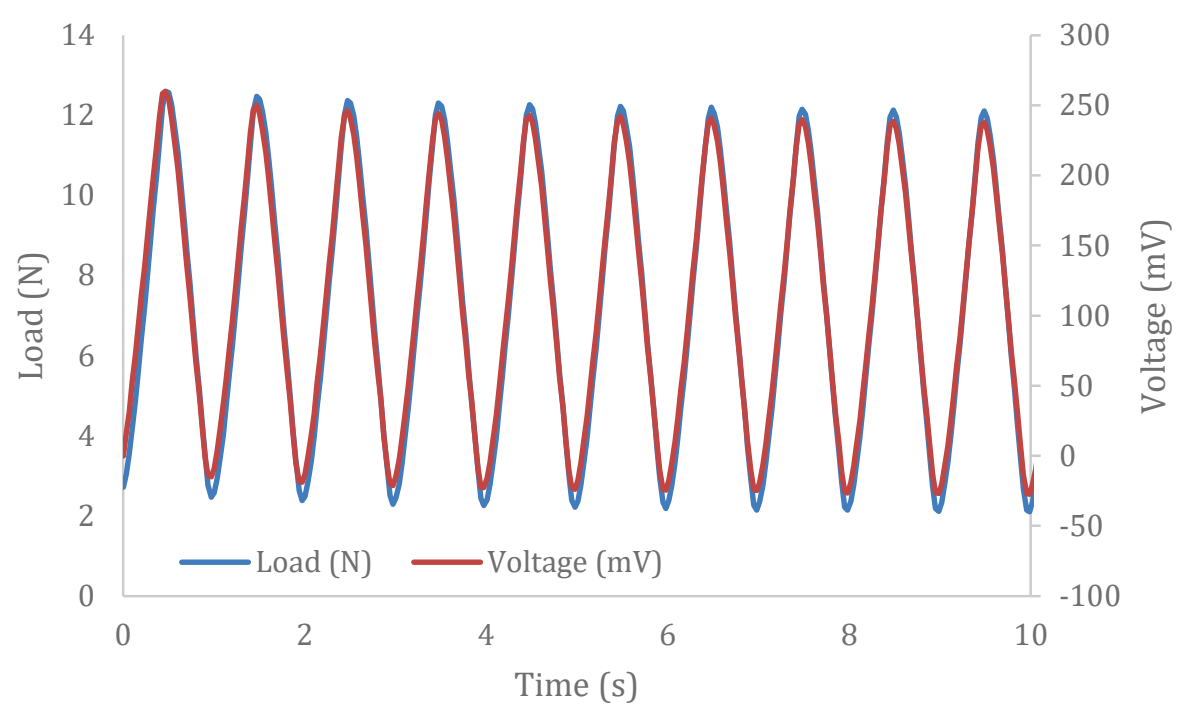

(b)

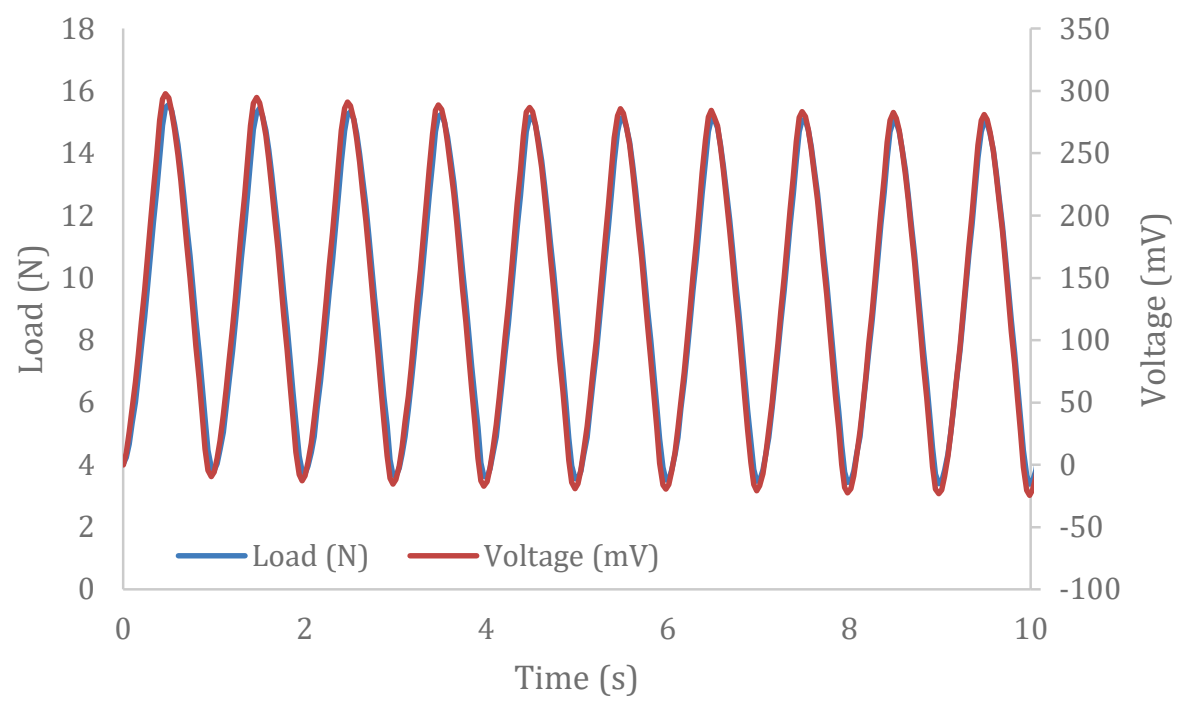

Figure 2.16 Load sensing performance (a) before and (b) after 10,000 cycle fatigue test. There is no significant change in sensing performance between these two cases $(t=-0.685, p=0.501)$.

\subsection{Cytotoxicity}

Due to the novel design of the fixation plate, a preliminary cytotoxicity test was performed on the parylene-coated fixation plates using MC3T3-E1 sub-clone 14 osteoblasts 
(American Type Culture Collection, Manassas, VA, USA). Culture media was made by adding $10 \%$ by volume FBS (Sigma Aldrich, St. Louis, MO, USA) and $0.5 \%$ by volume Pen-Strep (Sigma Aldrich, St. Louis, MO, USA) to MEM- $\alpha$ (Gibco ${ }^{\text {TM }}$ A1049001). Fixation plates $(n=3)$ were added to vials of $30 \mathrm{~mL}$ of cell culture media. $10 \mathrm{~mL}$ of media was removed at 24 and 72 hours and added to the osteoblasts. 24 hours after the addition of the media, calcein-AM (Sigma Aldrich, St. Louis, MO, USA) and ethidium homodimer-1 (Sigma Aldrich, St. Louis, MO, USA) stains were added to the cultures and the cultures were imaged to observe cell death.

A cell viability test was also performed by exposing fixators $(n=3)$ to MEM- $\alpha$ culture media with $10 \%$ by volume FBS and $0.5 \%$ by volume Pen-Strep. The media was exposed to the fixators for 24,48 , and 72 hours, and control media was stored with them in the same incubator at $37^{\circ} \mathrm{C}$ with $5 \% \mathrm{CO}_{2}$. At the end of the 24,48 , and 72 hour intervals, the media was removed and added to MC3T3-E1 sub-clone 14 osteoblasts in 96 well plates and allowed to grow in an incubator at $37^{\circ} \mathrm{C}$ with $5 \% \mathrm{CO}_{2}$ for 24,48 , and 72 hours. At the end of this time, CellTiter-Blue ${ }^{\circledR}$ cell viability assay (Promega, Madison, WI, USA) was introduced to the cells and imaged 4 hours later.

Sample images from the live/dead assay are shown in Figure 2.17. These results are shown quantitatively in Figure 2.18 and Table 2.2. A t-test shows a significant increase $(t=-2.230$, $p=0.050)$ in the relative abundance of live cells in the experimental group $(\mu=99.53 \%, \sigma$ $=0.30 \%)$ relative to the control $(\mu=98.86 \%, \sigma=0.67 \%)$. However, there is no decrease in the ratio of live cells to the total number of cells. 


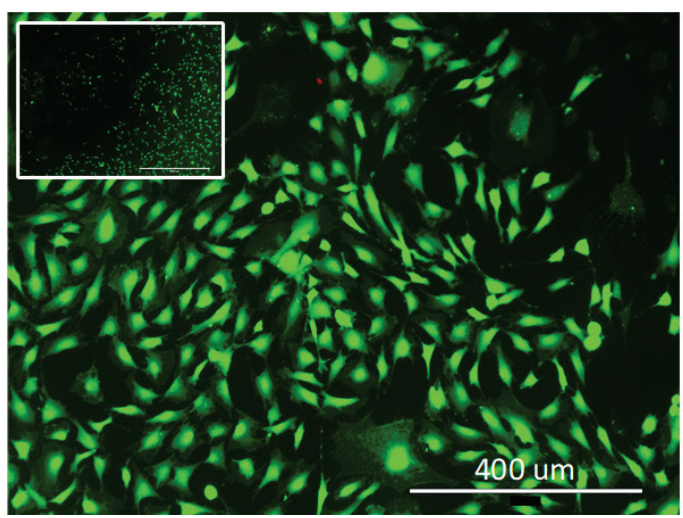

Control, 24 hours

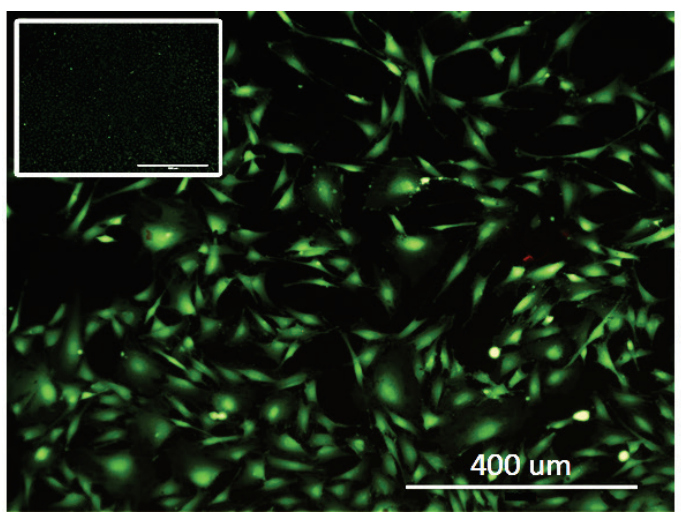

Control, 72 hours

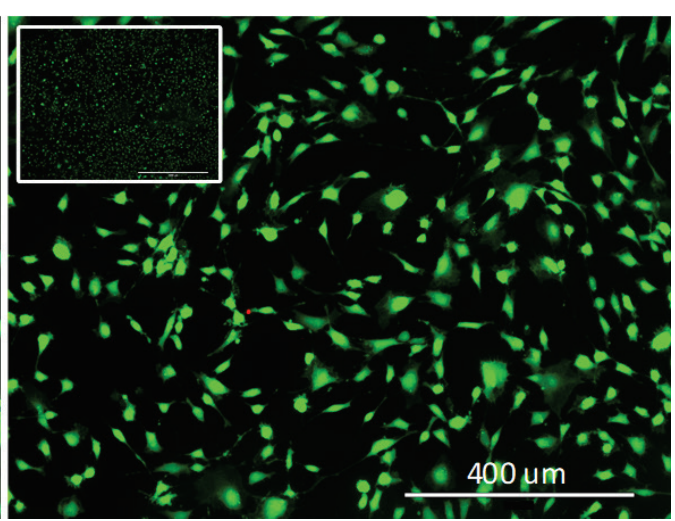

Experimental, 24 hours

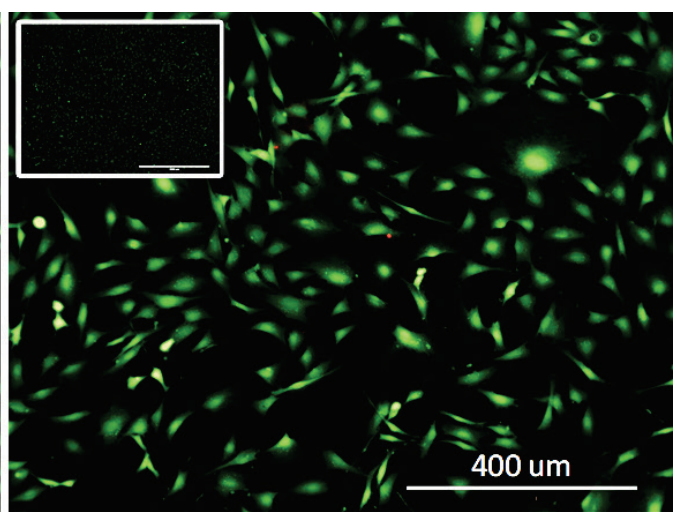

Experimental, 72 hours

Figure 2.17 Results of the cytotoxicity test at 24 and 72 hours at 10x magnification (inset at 4x magnification). Calcein-AM (green) shows live cells and ethidium homodimer-1 (red) shows dead cells. 


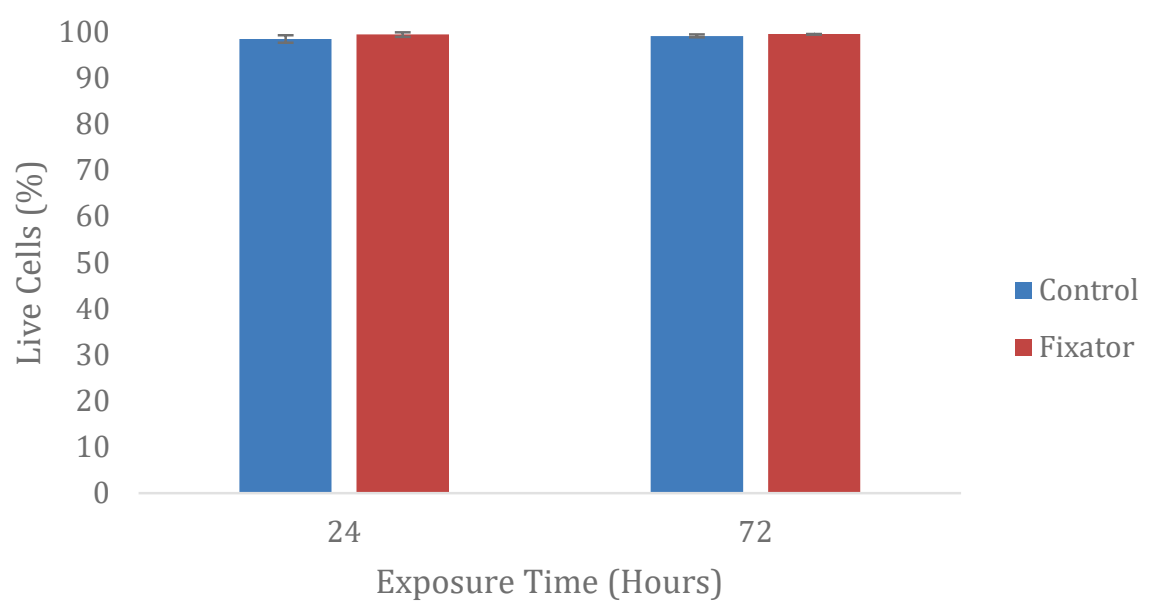

Figure 2.18 Percentage of live cells relative to the number of total cells for media with and without exposure to fixator $(t=-2.230, p=0.050)$.

Table 2.2 Live/dead assay cell counts.

\begin{tabular}{|ccccccc|}
\hline \multicolumn{7}{c|}{ Control } \\
Sample & Live & Dead & Ratio & Live & Dead & Ratio \\
1 & 128 & 3 & 0.977 & 321 & 2 & 0.994 \\
2 & 132 & 2 & 0.985 & 216 & 2 & 0.991 \\
3 & 312 & 2 & 0.994 & 202 & 1 & 1 \\
& & \multicolumn{72}{c}{ Hour Exposure } & & \\
1 & 271 & 3 & 0.989 & 266 & 1 & 0.996 \\
2 & 417 & 2 & 0.995 & 240 & 1 & 0.996 \\
3 & 117 & 1 & 0.992 & 194 & 1 & 0.995 \\
\hline
\end{tabular}

The results of the cell viability assay are shown in Figure 2.19. A t-test shows no significant difference $(t=-1.147, p=0.253)$ in fluorescence between osteoblasts exposed to the fixator media $\left(\mu=19.0 \times 10^{6}, \sigma=10.8 \times 10^{6}\right)$ compared to those exposed to the control media $(\mu=$ $\left.17.4 \times 10^{6}, \sigma=8.5 \times 10^{6}\right)$. This indicates that there is no statistically significant difference in cell viability within 3 days due to exposure to the fixator. 

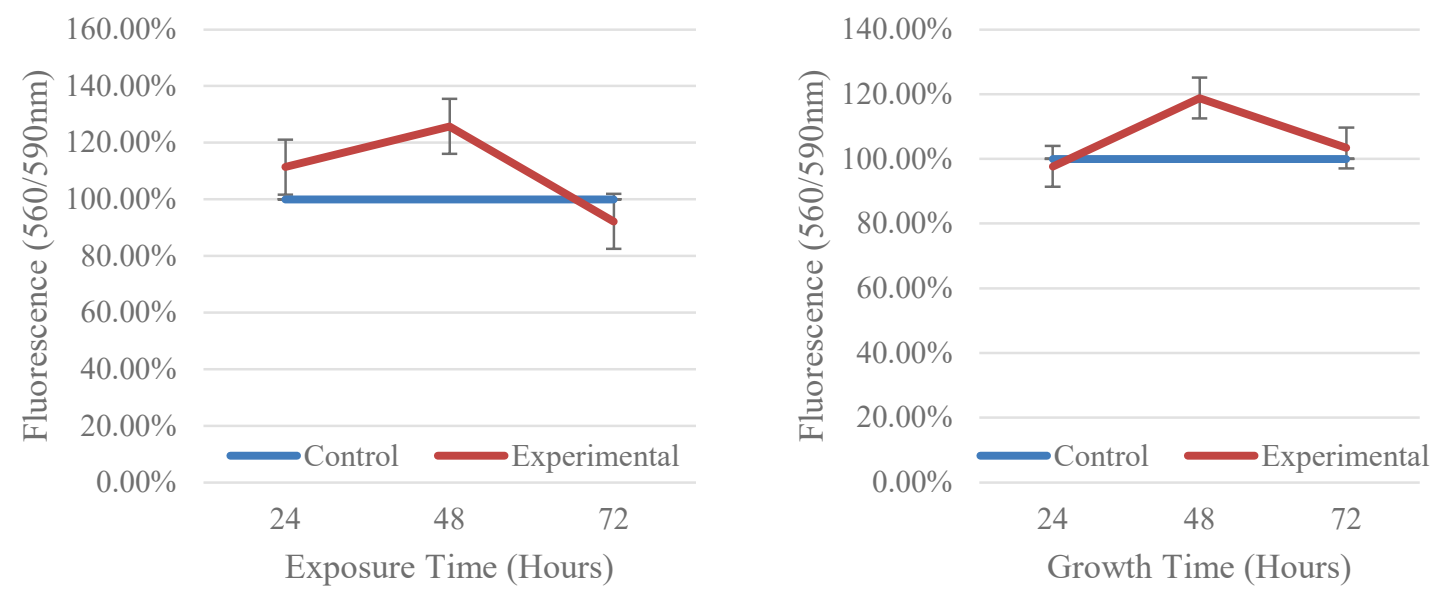

(c)

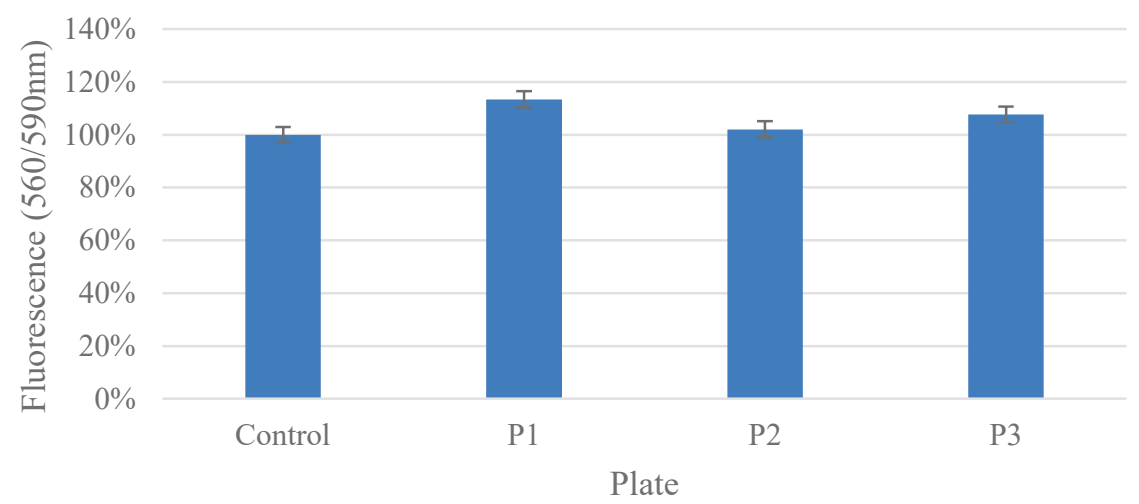

Figure 2.19 Fluorescence of cells exposed to fixators relative to the control media over (a) time exposed to fixators and over (b) time media is exposed to cells. (c) The average fluorescence of each plate relative to the control group.

\subsection{Conclusion}

The actuation sensitivity of the fixation plate described in this work is roughly $126 \mathrm{~nm} / \mathrm{V}$. This is somewhat lower than the calculated sensitivity of $167 \mathrm{~nm} / \mathrm{V}$. The difference is likely due to imperfect transmission of displacement from the piezoelectric actuator through the endcaps to the bone. It appears that, in the current design, approximately $75 \%$ of the 
displacement occurring within the plate is transferred through the screws to the bone, likely due to the elasticity of the polymer endcaps and off-axis nature of the load application.

The load monitoring sensitivity of this plate matches the values provided by the manufacturer exactly. Both values are roughly $25 \mathrm{mV} / \mathrm{N}$. The difference in efficiency during actuation and load monitoring may again be due to how the off-axis loads are transferred. During load monitoring, the off-axis loading results in some bending, but the entirety of the load is transmitted through the plate before the bone begins to heal.

The piezoelectric fixation plate described in this work is able to apply cyclic loading in the LMHF regime at $0.7 \mu \mathrm{m}$ and $31.25 \mathrm{~Hz}$ during the early healing phase before bridging has occurred. Based on previous research, this regime should be beneficial to the speed of soft callus formation. Both the frequency and amplitude of the actuation may be modified based on the signal supplied to the fixation plate, making this plate a versatile tool in the investigation of the use of LMHF actuation on fracture healing. The magnitude of the actuation decreases as the fracture heals due to increased blocking forces, which promote the formation of bone once the callus has bridged. By investigating when to begin and end actuation therapies, this tool may be useful in improving the scientific understanding of fracture healing.

The fixation plate is also able to function as a load monitoring device to observe the progress of healing quantitatively. As the callus stiffens, the loading sensitivity decreases so that the same loading pattern present during animal ambulation will result in decreasing voltages as the callus stiffens. This requires further investigation in vivo in order to observe how closely the differential voltage corresponds to fracture strength, but similar strain gauge technology has been used clinically for several decades and shown promising results.

While piezoelectric materials have several non-linear behaviors such as creep, hysteresis, and drift; these pose only minor problems in this application. Cyclic actuation and loading profiles significantly reduce the importance of creep and hysteresis when compared with precision sensing and actuating applications. Drift may pose a problem, but it typically 
remains within a $\pm 2 \mathrm{~V}$ range from a $0 \mathrm{~V}$ offset in testing, which means that is remains within the range of a typical microcontroller ADC. 


\section{Telemetry Platform}

\subsection{Overview}

The telemetry platform described in this work has two primary roles: to provide voltage signals for actuation of the fixation plate, and to provide a load monitoring system for the fixation plate. It serves as a communication node between the user and the internal fixation

plate. Through the means of the telemetry unit, the user may start and stop actuation and sensing on command. The loads monitored are also measured by the telemetry unit and relayed to a computer.

This device contains several major sub-systems (Figure 3.1). The interface between the telemetry platform and the piezoelectric fixation plate features two parallel pathways, one for actuation and one for sensing. These pathways may be enabled or disabled using onboard solid-state photorelays. The bidirectional radio communication is compliant with the Bluetooth 4.0 standard and utilizes the Bluetooth Low Energy (BLE) protocol. Both the radio and the piezoelectric interface are controlled with an 8051 microcontroller. Power is supplied to the telemetry platform via a regulated lithium-polymer battery. This battery is recharged using inductive wireless power transfer. 


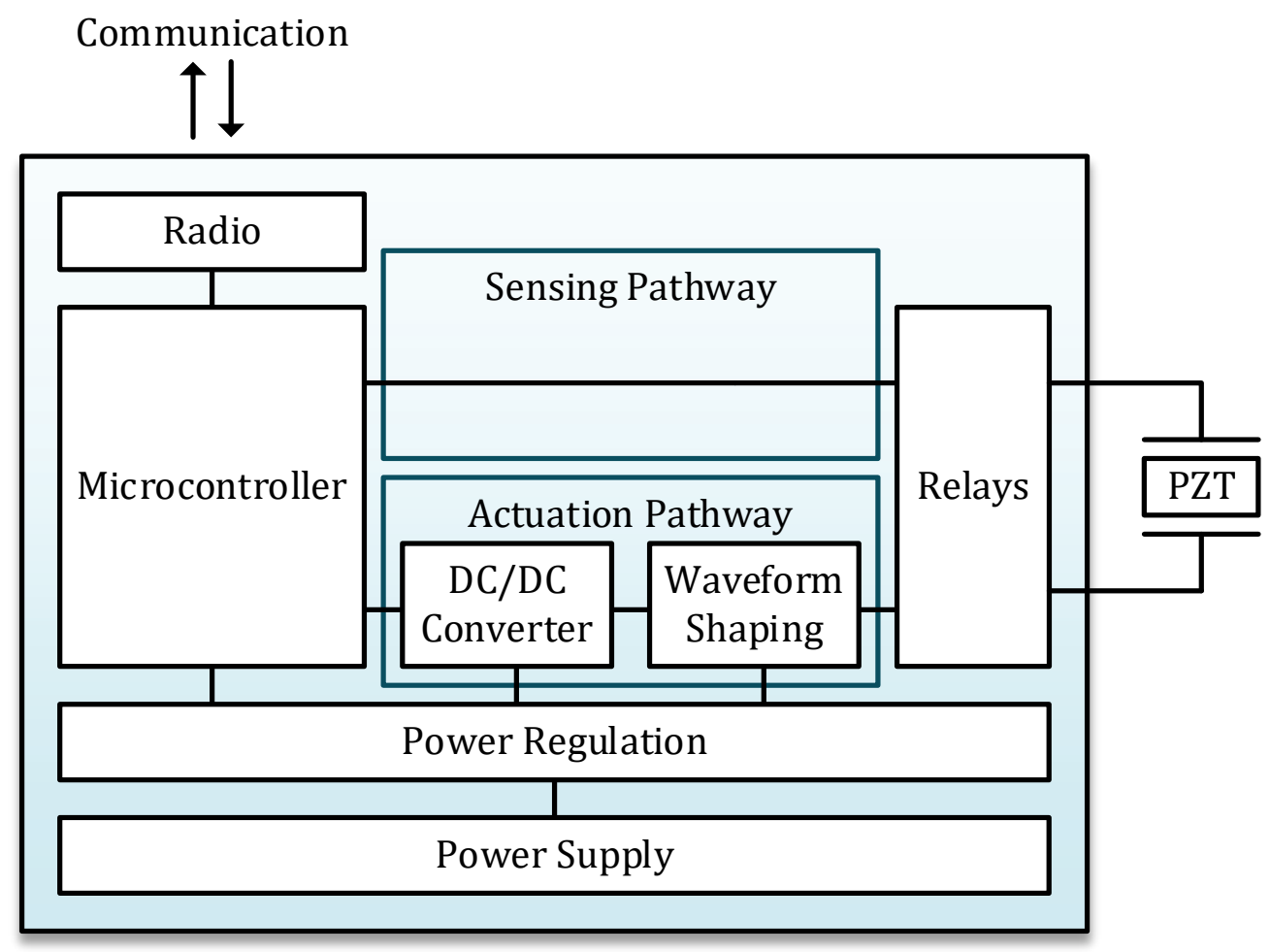

Figure 3.1 Block diagram showing the major functions of the telemetry platform described in this work.

The telemetry unit presented in this chapter can either provide voltages to generate strains in the fixation plate or monitor the voltage of the fixation plate for load monitoring. It uses relays to switch between actuating and sensing modes and can disable both modes when not in use. Actuation and sensing mode cannot be used concurrently. The actuation pathway is able to generate voltages of up to $200 \mathrm{~V}$ at up to $1992 \mathrm{~Hz}$. The sensing pathway is able to measure voltages from $-2.5 \mathrm{~V}$ to 2.5 with rollover for negative values. Bluetooth Low-Energy is used to enable wireless communication for control and data logging, and this communication system is able to transmit through tissue. A $400 \mathrm{mAh}$ battery is used to provide up to 1 hour of constant actuation or up to 72 hours of constant sensing performance. A wireless recharging system is implemented to recharge the battery through the body tissue. 


\subsection{Bluetooth Communication}

Bluetooth technology is growing rapidly for many different uses including audio streaming, data transfer, location services, and device networking [77]. The Bluetooth Low Energy, or BLE, protocol is aimed at low-power data transfer applications such as implantable medical devices. The foundation of the telemetry system for this work is the Bluegiga BLE113 wireless module (Silicon Labs, Austin, TX). This module combines an 8051 microcontroller with a Bluetooth 4.0 compliant radio [78].

\subsubsection{Pairing and Connectivity}

In version 4 of the Bluetooth Low Energy protocol, communication primarily occurs between sensor pairs (Figure 3.2). Several pairs of roles are used to describe how the devices in these pairs relate to each other in different ways and at different times. On startup, Bluetooth devices are unpaired. and several roles are used to describe their behavior in the pair. When receiving power, Bluetooth devices are unpaired (Figure 3.2a). An unpaired device called a peripheral advertises itself by repeatedly transmitting packets of information, often including its name, address, and some of its functionality, to any nearby devices. Nearby devices called central devices, usually a phone or computer, act as scanners and will read these packets. These packets can be parsed, and the scanner can then initiate a connection with a connectable nearby device. The devices are paired once this connection is formed (Figure 3.2b), and the central becomes the master and the peripheral becomes the slave. 
(a)

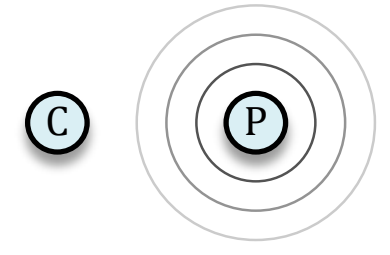

(b)

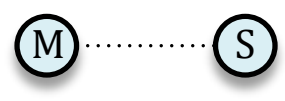

Figure 3.2 (a) Unpaired central (C) and peripheral (P) devices. The peripheral device transmits packets until a connection is formed. When the devices are paired (b) they become the master (M) and slave (S) respectively.

The telemetry unit described in this work has the roles of the peripheral and slave devices. It advertises its name, address, and some of its functions so that it can be identified as the telemetry for the piezoelectric fixation plate. The central/master device used in this work is the BLED112 (Silicon Labs, Austin, TX), a USB hub that interfaces with software that will be discussed in Section 3.5.2.

\subsubsection{Signal Propagation}

To observe the effect of path loss an attenuation on the maximum transmission range of the transceiver used in this work, the TI CC2540 Dongle was used in conjunction with the SmartRF Protocol Packet Sniffer (Texas Instruments, Dallas, TX, USA) to detect BLE packets at varying distances from an advertising transmitter. Testing was performed in an enclosed environment representative of what would be present in many animal testing facilities at three roughly eight-hour intervals. The BLE113 was packaged in a watertight 3D-printed enclosure and placed in air, inside a small (5 $\mathrm{cm}$ diameter) beaker filled with tap water, and surgically implanted into the abdominal cavity of adult Sprague-Dawley rat cadavers $(n=3)$. The packet sniffer was used to detect the advertising packets sent by the transceiver at varying distances until no more packets were received. The transmission range was identified as the distance at which no more packets were received. 
Distance is not the only factor in reliable wireless communication, and many communication errors are induced by other factors. Various propagation effects are present in most practical environments. Material boundaries such as walls introduce errors into signal transmission due to factors such as reflection, scattering, and diffraction [79]. It is also increasingly common to have other wireless signals operating in the same frequency range, such as Wi-Fi, Bluetooth, and other similar communication standards. As signals overlap, the number of transmission errors increase. Signal power also decreases over distance so that there is a maximum signal radius for a transceiver.

The same packet sniffer was used again to observe the errors introduced by the environment. Packets received from the transmitter-once again located in air, a beaker of water, and implanted in a rat abdominal cavity — were compared with the expected contents of the packets to quantify the byte error rate. This error rate was then multiplied by the number of bytes in these packets to obtain a relative measure of the number of packets containing errors.

The results of the signal propagation experiments are shown in Table 3.1. In both air and water, the packet sniffer was able to detect packets through the full length of the room. When the signal was attenuated by placing the transmitter in the abdominal cavity of a rat cadaver, the transmission range varied more. Sometimes the sensor was detected across the full length of the room, sometimes the sensor could not be detected only 5 meters away. Many factors influence transmission range, such as transmitter power, receiver sensitivity, and the relative noise level. Therefore, these results do not constitute a de facto maximum range. Instead, they show the relative effects of the environment. By placing the transceiver behind a roughly $1 \mathrm{~mm}$ wall of tissue, the transmission range dropped to roughly $70 \%$ of its performance in air. Therefore, there are often other considerations present in implantable sensors that are not required in wearable sensors. 
Table 3.1 Approximate transmission range of the telemetry unit described in this work. In the water trial, the sensor was packaged and submerged in a container filled with tap water. In the tissue trial, the sensor was implanted in the cadaver of an adult Sprague-Dawley rat.

\begin{tabular}{|cc|}
\hline Transmitter & Transmission \\
Location & Range $(\mathrm{m})$ \\
Air & $>20$ \\
Water & $>20$ \\
Tissue & $5->20$ \\
\hline
\end{tabular}

One major effect of path loss and attenuation is the inability to maintain a connection between two devices. This effect, commonly known as link loss, can be a significant problem in implantable devices. In Bluetooth, the control of the link is defined in the Logical Link Control and Adaptation Layer Protocol (L2CAP, [80]). the slave device (the device that is being controlled) is required to send updates to the master (the device which controls the other device) within a brief time window at regular intervals. If the slave device fails to send an update during several successive connection intervals, the connection will be lost.

The expected trend due to path loss and attenuation is visible in Figure 3.3. More errors are present at greater distances. However, the number of errors varies much more significantly within trials than between trials. This effect is magnified in more lossy media such as tissue and water. This is due to the effects of interference, whether those present in the environment by other Bluetooth and Wi-Fi devices or those present due to multipath effects such as reflection. Two conclusions can be inferred from this experiment. (1) In general, tissue has the most packet loss, while air has the least loss. (2) In this noisy testing environment, the effect of time and location had a much more significant effect than distance. For example, the test in tissue at $1 \mathrm{~m}$ had more byte errors than packets. 


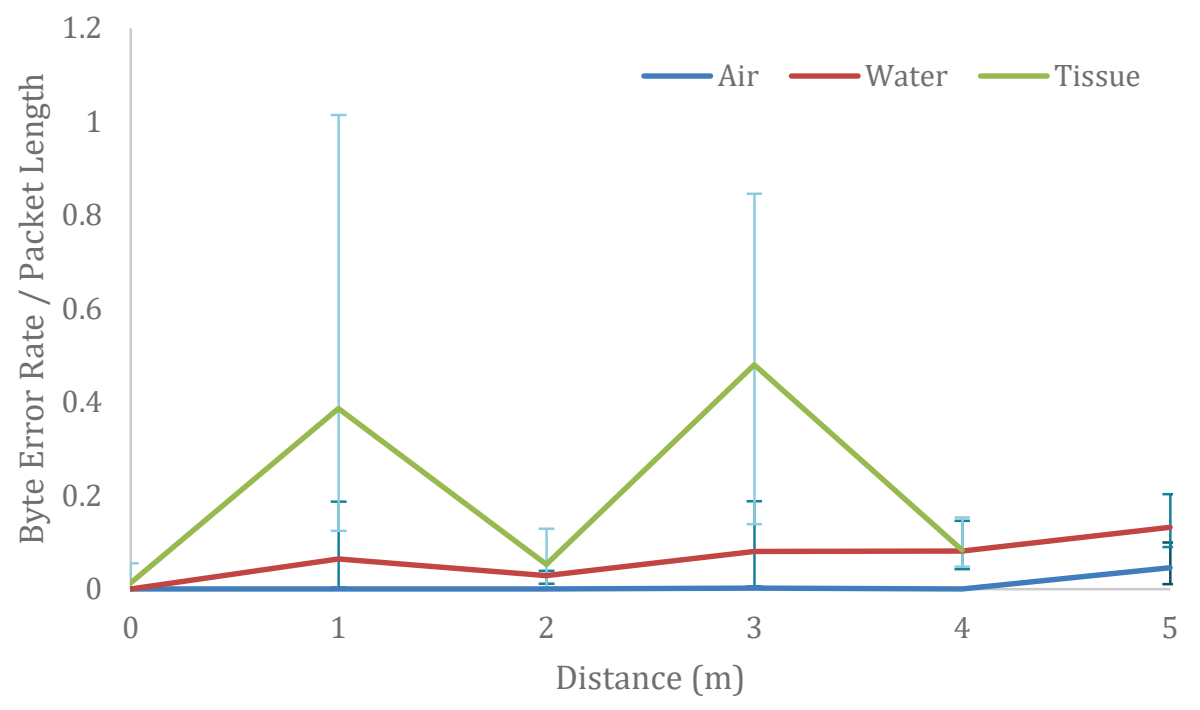

Figure 3.3 Relative error rate of received signals at different distances in different media. Error rate is quantified as the byte error rate relative to the total packet length. There is much greater variance in error rate within trial conditions than between trial conditions.

\subsubsection{Data Storage}

Data is stored locally on a single device known as the server in the client/server role pair. In this work, the implantable sensor always acts as the server. The client device can receive pushed data from the server, ask the server for specific information, or initiate changes to the server. Data is stored hierarchically using blocks called attributes: a data structure containing an address called a UUID (universally unique identifier), properties describing things like read/write access, and a payload. Each Bluetooth server has a single generic attribute (GATT) profile, which one or more services, which in turn contain one or more characteristics. A service contains a group of related device features, so that a device may have a battery state service and an actuation service. Characteristics expose a state of the device, so the battery state service may have characteristics to report the battery voltage, whether it is charging or not, and even charge current.

The GATT profile used by this device is shown graphically in Figure 3.4. 


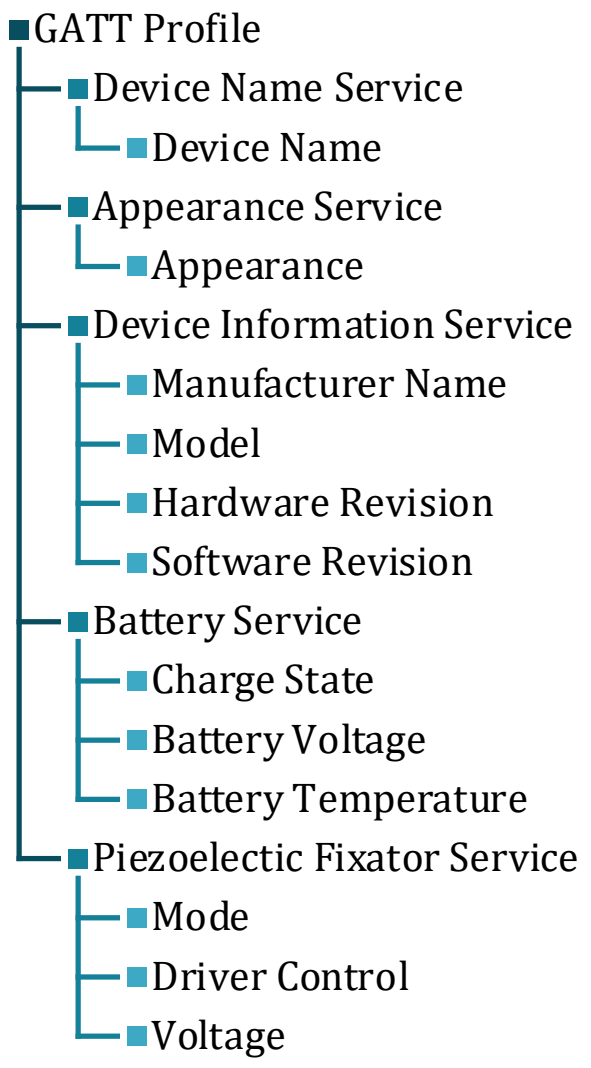

Figure 3.4 The GATT (Generic Attribute) profile used by the device described in this work.

The Device Name Service simply contains the name of the device, which is provided via software to easily identify the device. The name provided is included in the data packets that are advertised by unpaired devices.

The Appearance Service is required in BLE 4.0 and serves as an easy way to identify the device as a specific class of device, such as a heart rate monitor or thermometer. Since this device does not fall into any of the official categories, its appearance is unknown.

The Device Info Service provides further information about the device, such as the manufacturer and model of the device. It also contains the hardware and software information numbers to make troubleshooting easier when the device is inaccessible due to implantation. 
The Battery Service used in this profile is slightly different than the one provided by the Bluetooth SIG. To facilitate wireless charging, the charge state of the battery may be used to identify if the battery is charging, or as an indirect measure of current by reading the voltage that is being pulled low by the battery charger. The voltage level of the battery is also reported. To improve safety of the device, the temperature can also be reported. Using external software to control the wireless charging, wireless power transfer can be stopped if temperature levels exceed safe levels (see section 3.4.5).

The Piezoelectric Service provides access to the functions relating to the piezoelectric fixation plate. The mode of the device may be controlled so that it can be in actuation mode, sensing mode, or low-power mode. The actuation driver may also be controlled here by changing the voltage and frequency of the actuation. In addition, the actuation can be started for 20 minutes or stopped on command. When in sensing mode, the voltage of the actuator is reported here and pushed to the client device.

\subsection{Piezoelectric Interface}

The interface with the piezoelectric fixation plate is composed of two parallel pathways for actuation and sensing. These pathways operate asynchronously. In other words, load monitoring cannot be performed at the same time as actuation.

\subsubsection{Actuation Pathway}

There are two major subsystems that are present in this actuation pathway: a DC/DC converter and a waveform generator. Piezoelectric actuators are well known for requiring high voltages to generate modest actuation. Because of this, a DC/DC converter will be used to generate a sufficiently high voltage while still using a small, rechargeable battery.

Various topologies of DC/DC converters exist. Figure 3.5 shows some of the common topologies of switching converters where the output voltage is greater than the input voltage that were considered for this design. In a boost converter (Figure 3.5a), current is 
stored in the inductor when the switch is closed. When the switch is open, the inductor provides current through the load. The output voltage is determined by factors such as the switching frequency and inductance. The major limitation of this design is that the input and the output are not isolated, which can lead to possible damage in high voltage applications.
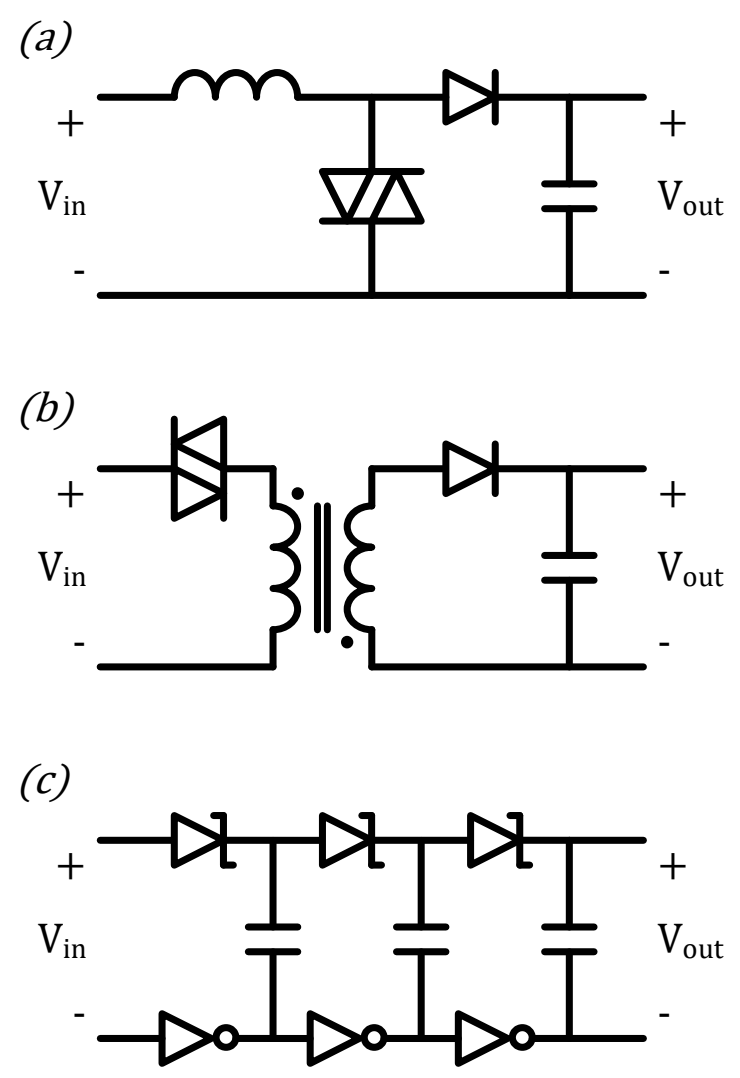

Figure 3.5 Common topologies for switched DC/DC converters considered in this design: (a) boost converter, (b) flyback converter, and (c) charge pump.

The flyback converter (Figure 3.5b) is similar to the boost converter, except it features a transformer in place of the inductor. This provides two significant benefits. First, the transformer allows the output voltage to be isolated from the input voltage, significantly reducing the risk of damage due to the high generated voltages. In addition, by varying the number of turns on the transformer, higher voltages may be generated. However, transformers tend to be significantly larger than inductors. 
The charge pump (Figure 3.5c) features a series of diodes and capacitors. During the switching cycle, voltage is stored in the capacitors equal to the voltage drop across the diode. This provides a simple, inductorless design. However, due to the high number of individual capacitors needed to generate high voltages, charge pumps are not often used to generate voltages significantly higher than the source voltage, and the size of the resulting converter can be very big. Due to the size requirements of this device, the boost converter was selected to avoid the addition of large components.

The second component in the actuation pathway chain is the generation of the target waveform. A digital-analog converter (DAC) may be used to generate an arbitrary waveform, which can be smoothed using capacitors. In this design, a sine wave is the target waveform, so there is no need for a complicated waveform generator.

The final design selected for this device integrate both a boost converter and waveform shaper into one integrated circuit (Figure 3.6). The TI DRV2667 (Texas Instruments, Dallas, TX, USA) is able to generate voltages up to $200 \mathrm{~V}$ and can create arbitrary waveforms. While it requires several large peripheral components, such as inductors and capacitors, the full actuation pathway can fit in roughly $1 \mathrm{~cm}^{2}$. The output voltage is defined by resistors, and $\mathrm{I} 2 \mathrm{C}$ communication is used to configure and control the waveform generation. 


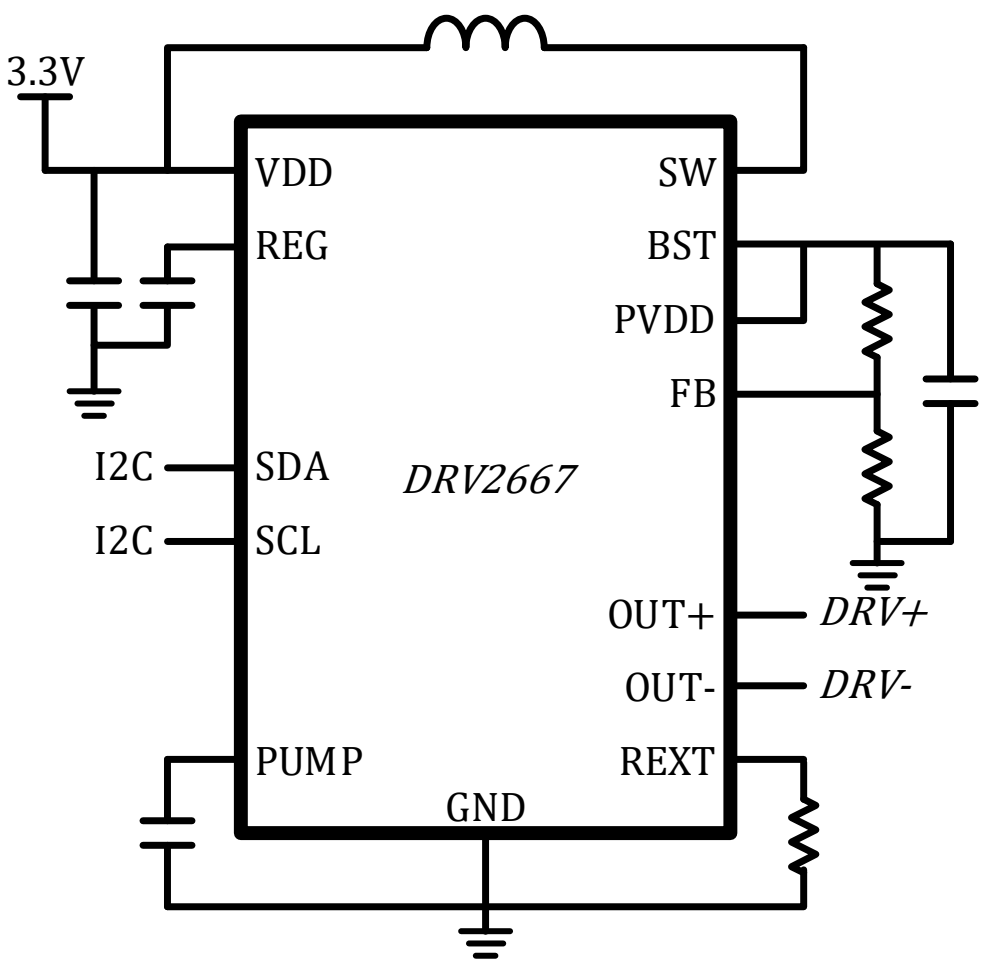

Figure 3.6 Actuation pathway. The TI DRV2667 generates voltages large enough to actuate the piezoelectric stack and can generate arbitrary waveforms.

\subsubsection{Sensing Pathway}

Piezoelectric sensors operate very differently than strain gauges, which have been more commonly used in similar load-monitoring applications. Because of this, the sensing pathway is significantly different than what is found in similar works [29]. Conventional strain gauges are resistive elements. When they experience strain along the sensing direction, the wires in the gauge are stretched or compressed, resulting in a changed overall resistance. The sensitivity of a strain gauge is commonly identified as a gauge factor, a ratio of change in resistance per change in strain:

$$
G F=\frac{\Delta R / R}{\varepsilon}
$$

By this definition, a strain gauge changes in resistance as it experiences strain. Therefore, the sensing pathway for a resistive strain gauge typically includes a Wheatstone bridge, 
which converts this change in resistance into a measurable change in voltage. The Wheatstone bridge also helps to account for changes in resistance due to non-strain factors, such as changes in environmental temperature and pressure. The resulting sensitivity is typically very low, especially when measuring microstrain movements present in the rat fixation model. For example, a similar fixation plate designed for use in Sprague-Dawley femoral defects has an unamplified sensitivity of roughly $0.280 \mathrm{~V} / \varepsilon$ (unpublished data from [81]). As a result, amplification is also required in order to reliably measure strain amplitudes on the order of 2000 to $6000 \mu \varepsilon$.

By contrast, the piezoelectric sensor used in this work has a sensitivity of roughly $25 \mathrm{mV} / \mathrm{N}$ (Section 2.4). The output of the piezoelectric sensor is quantified in terms of voltage in this work, though other authors prefer other quantities $[82,83]$. This output may be read by an $\mathrm{ADC}$ and digitally processed by a microcontroller or computer.

\subsubsection{Switching}

In order to switch between the actuating and sensing pathways, four solid-state photorelays were used (Figure 3.7). In order to operate in actuation mode, the piezoelectric element is connected to the two outputs of the DRV2667 piezoelectric driver. To operate in sensing mode, the one side of the piezoelectric element is connected to a 12-bit microcontroller ADC, while the other side is connected to ground. 


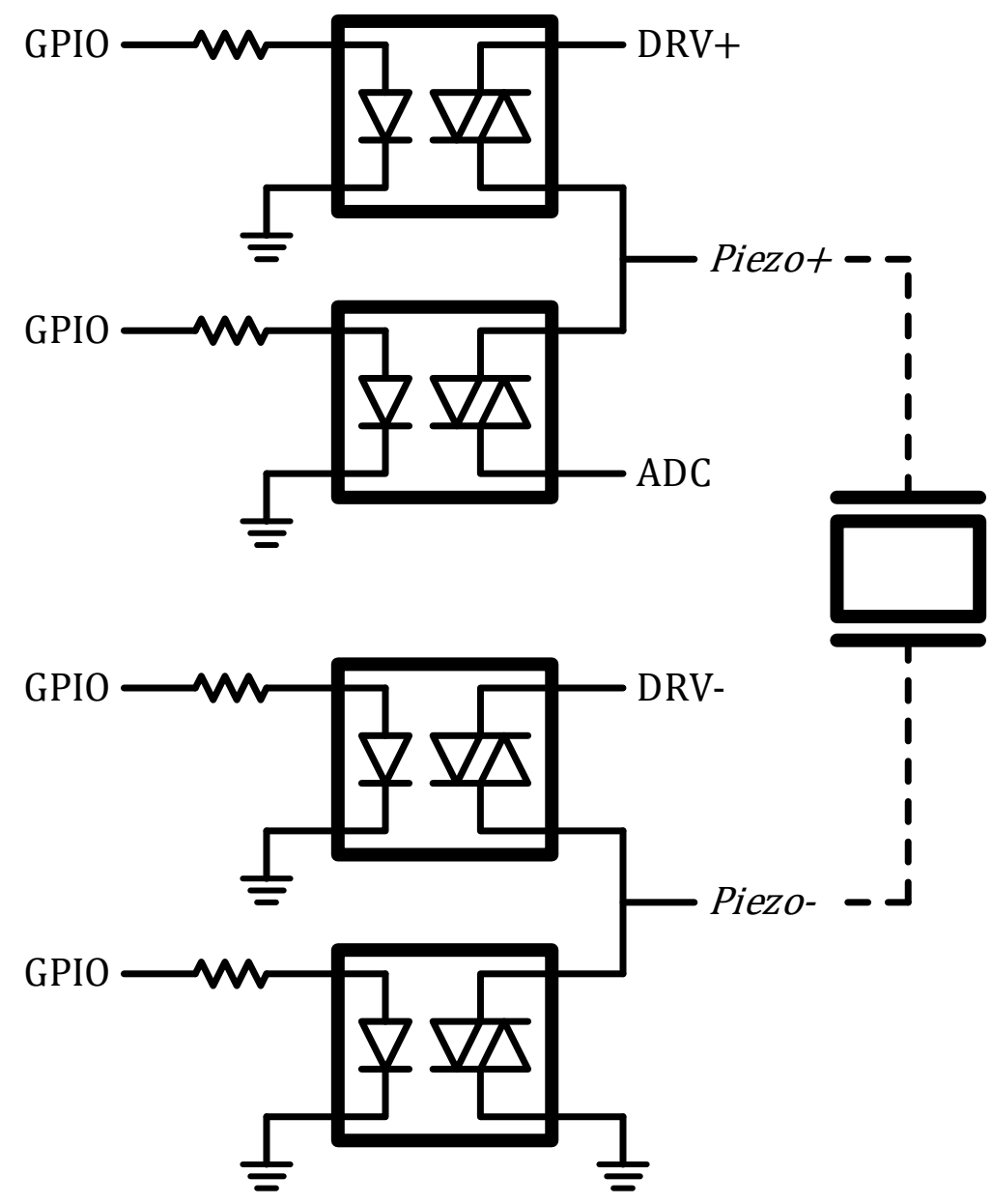

Figure 3.7 Schematic showing switching elements and their connections.

The photorelays selected for use in this device are the TLP3420 (Toshiba Corporation, Tokyo, Japan). These relays each consist of a single form A relay (single pole, single throw, normally open). This part was selected primarily due to the small physical size (VSON4 package) and large load isolation voltage (100 V).

An LED acts as the input device of a solid-state photorelay, such as the one used in this device. When sufficient current passes through the LED, the two output terminals will be connected with a relatively low resistance between them. The input current range specified in the datasheet is between 5 and $20 \mathrm{~mA}$, so a $470 \Omega$ resistor is placed in series with each relay input to minimize current consumption when the relays are on. When in the on state, there is approximately $8 \Omega$ resistance across the switched output. Due to the highly 
capacitive nature of piezoelectric elements, the voltage drop across this resistance will be negligible.

\subsection{Power Supply}

To monitor the course of fracture healing until bridging occurs, this sensor needs a lifetime of at least 8 weeks [17]. The power requirements of a sensor system, microcontroller, and wireless telemetry can easily be met using a non-rechargeable battery [81]. However, the current consumed by the boost converter in generating the voltage needed for actuation is significant. Therefore, a rechargeable system is required. This may be done using a number of battery charging systems, such as wireless battery charging or local energy harvesting. This system using inductive charging to transfer power to the implanted device without transcutaneous wires.

\subsubsection{Wireless Power Transfer}

Several commercial solutions currently exist to facilitate wireless power transfer between a transmitter and receiver device. Qi charging (Wireless Power Consortium, Piscataway, NJ, USA) is one of the most common solutions and is commonly implemented in many cell phones. This specification uses a pair of carefully tuned coil inductors, one transmitter coil and one receiver coil. The greatest limitation of this method is that the relative position of the receiver coil significantly impacts the quality of charging. The range of these chargers in the vertical direction is less than $1 \mathrm{~cm}$ in air, and must be tuned with the target distance in mind. Power transfer efficiency also drops off rapidly as the relative coil position is off axis in the horizontal direction.

Alternatives to inductive power transfer exist. Visible and near visible light charging has been demonstrated in vivo [84] and has seen growing popularity ex vivo in companies like Wi-Charge. The in vivo demonstrations have required careful orientation of the laser with respect to the implant, while newer products include features such as automatic receiver tracking. 
A loosely coupled inductive wireless power system (Figure 3.8) has been implemented in this design. The transmitter features a coil of 22 AWG copper wire (EIS Wire \& Cable, South Hadley, MA, USA) with approximately 125 turns. It has a cylindrical shape with an inner diameter of $30 \mathrm{~mm}$, an outer diameter of $60 \mathrm{~mm}$, and a length of $5 \mathrm{~mm}$. The receiver features a coil with 32 AWG copper wire (EIS Wire \& Cable, South Hadley, MA, USA) with roughly 290 turns. It has a rectangular shape with an inner size of $20 \mathrm{~mm}$ x $10 \mathrm{~mm}$, an outer size of $35 \mathrm{~mm} \times 25 \mathrm{~mm}$, and a length of $2 \mathrm{~mm}$.

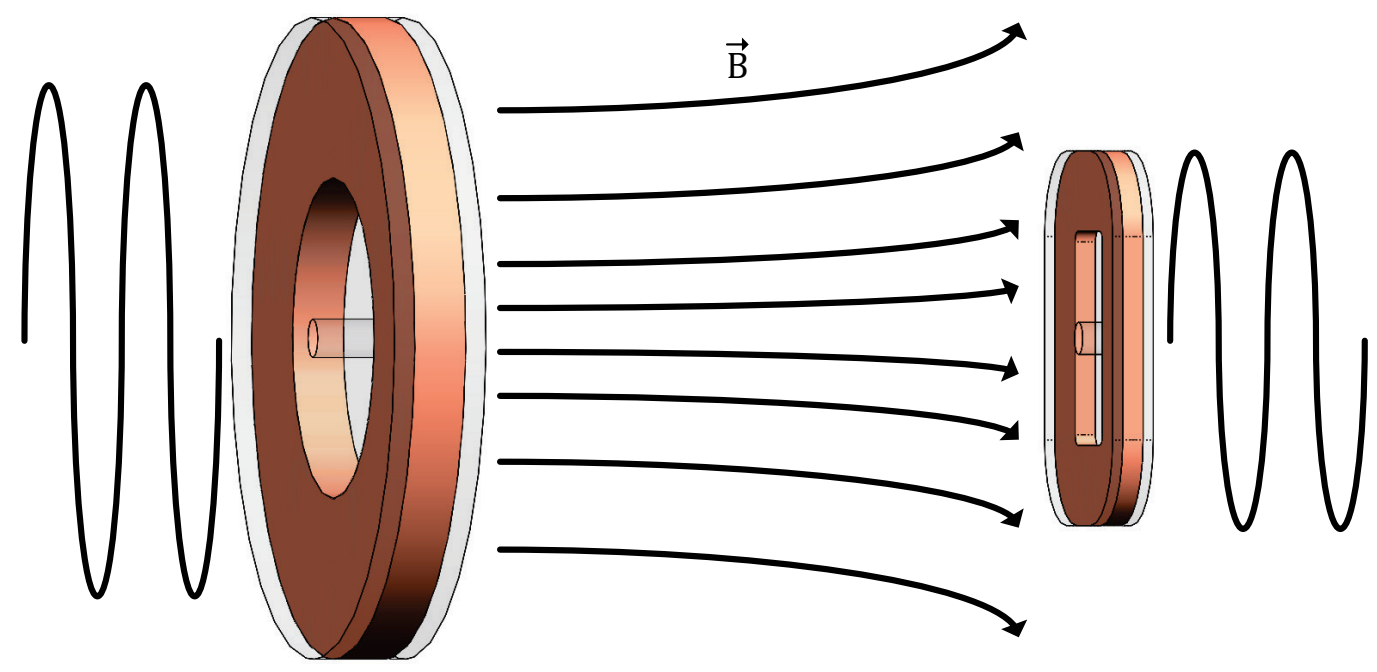

Figure 3.8 Loosely-coupled inductive wireless power transfer system. The transmitter coil used in this design is on the left; the receiver coil used in this design is on the right. A sinusoidal signal supplied to the transmitter coil generates a magnetic field. This magnetic field induces a sinusoidal current at the receiver coil.

The signal generation chain used in the transmitter is shown in Figure 3.9. A 20 Vpp, 1 kHz signal is generated by a Fluke $27110 \mathrm{MHz}$ Function Generator (Fluke Corporation, Everett, WA, USA), and can be controlled by a computer connected by a General Purpose Interface Bus (GPIB, IEEE 488.2) serial interface. The output of this is then amplified by a Tapco Juice J-1400 Power Amplifier. The gain of the amplifier can be adjusted by the user in response to the feedback provided by the sensor to generate enough power to charge the battery. This signal is then supplied to a coil inductor. 


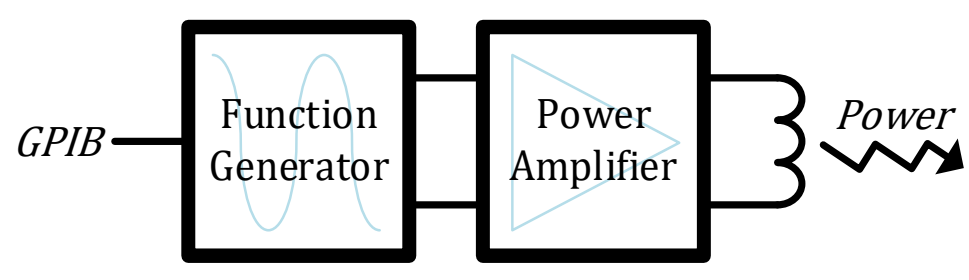

Figure 3.9 Schematic of wireless power transmitter. The signal generated by a function generator is amplified before being transmitted by a coil inductor.

The loosely-coupled coils do not operate in resonant mode. In tightly-coupled coils, the impedance of the coil decreases significantly at the resonant frequency, so the devices are carefully tuned to operate at resonance. In these coils, impedance increases near the resonance frequency (Figure 3.10). Therefore, it is desirable to operate far from the resonant frequency. 
(a)

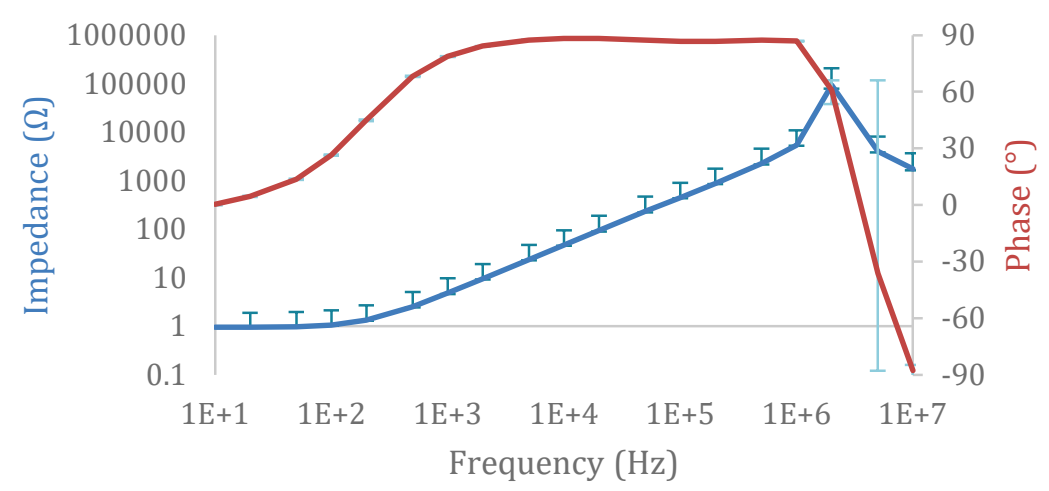

(b)

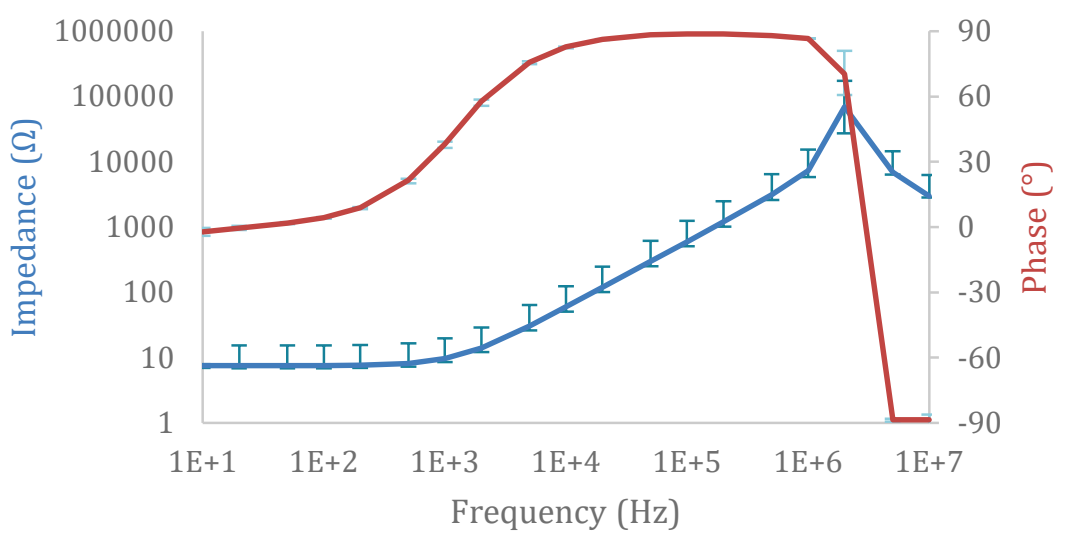

Figure 3.10 Impedance spectrum of both (a) transmitter $(\mathrm{n}=3)$ and $(\mathrm{b})$ receiver $(n$ $=3$ ) coils. Impedance significantly increases as the frequency approaches the resonant frequency for both coil designs.

As is the case in all wireless power transfer systems, the efficiency of wireless power transfer is significantly affected by misalignment. FEMM 4.2 (developed by David Meeker) was used to simulate the magnetic field generated around the transmitter coil by a 2 A current. A cross-sectional view of the coil and its field are shown in Figure 3.11. This simulation shows that the magnetic field decreases exponentially as distance along the $\mathrm{Z}$ axis increases, while the field strength is more stable radially across the diameter of the transmitter coil. 


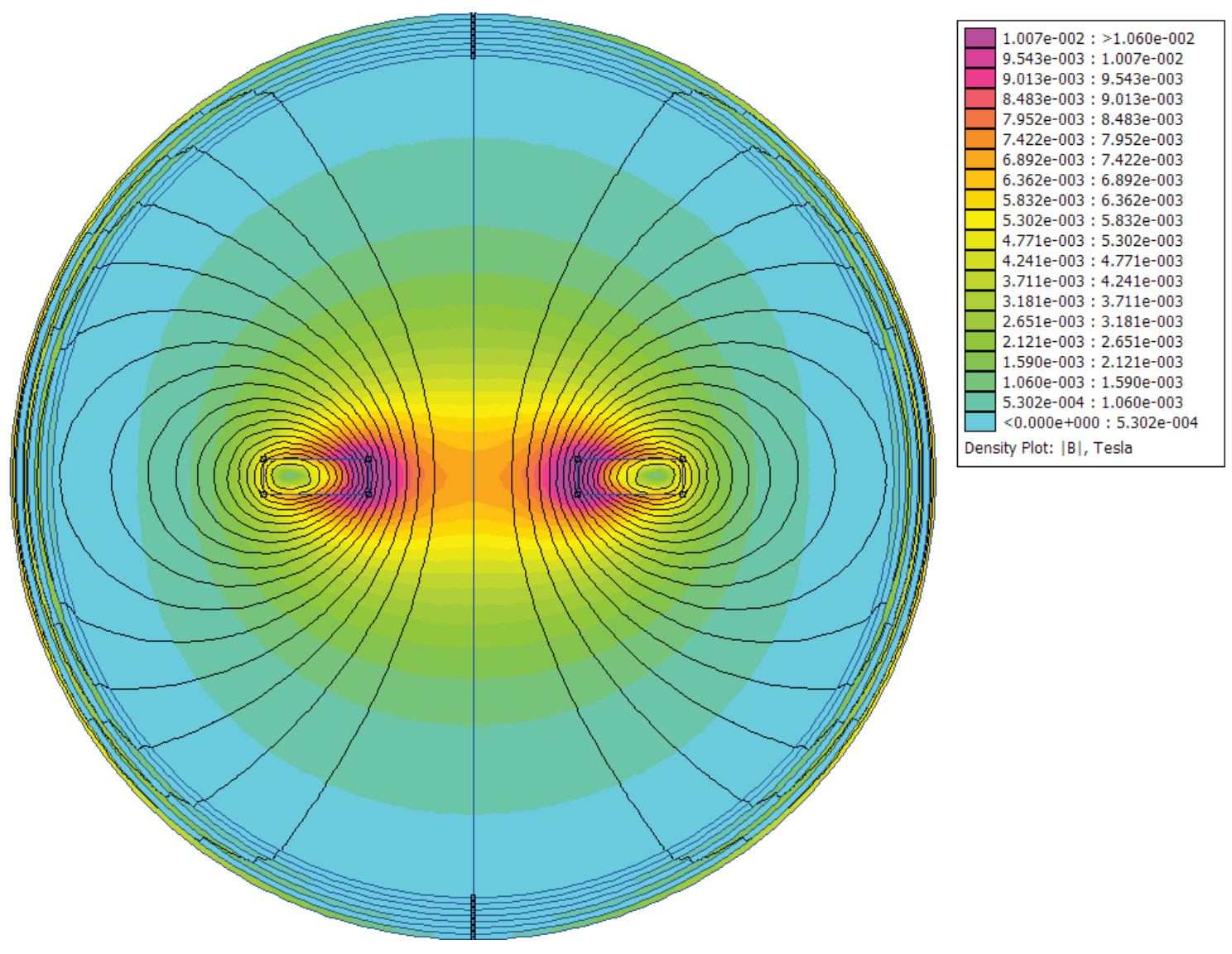

Figure 3.11 Cross-sectional view of the simulated flux density of the magnetic field generated by the transmitter coil at 2 A current.

The directionality of the magnetic field may be improved by the addition of a ferrite shield to the coil design. This restricts the passage of the magnetic field in one direction, resulting in improved magnetic field strength in the opposing direction. An additional simulation was run with the introduction of a ferrite shield to the transmitter coil. The resulting magnetic field is shown in Figure 3.12. 


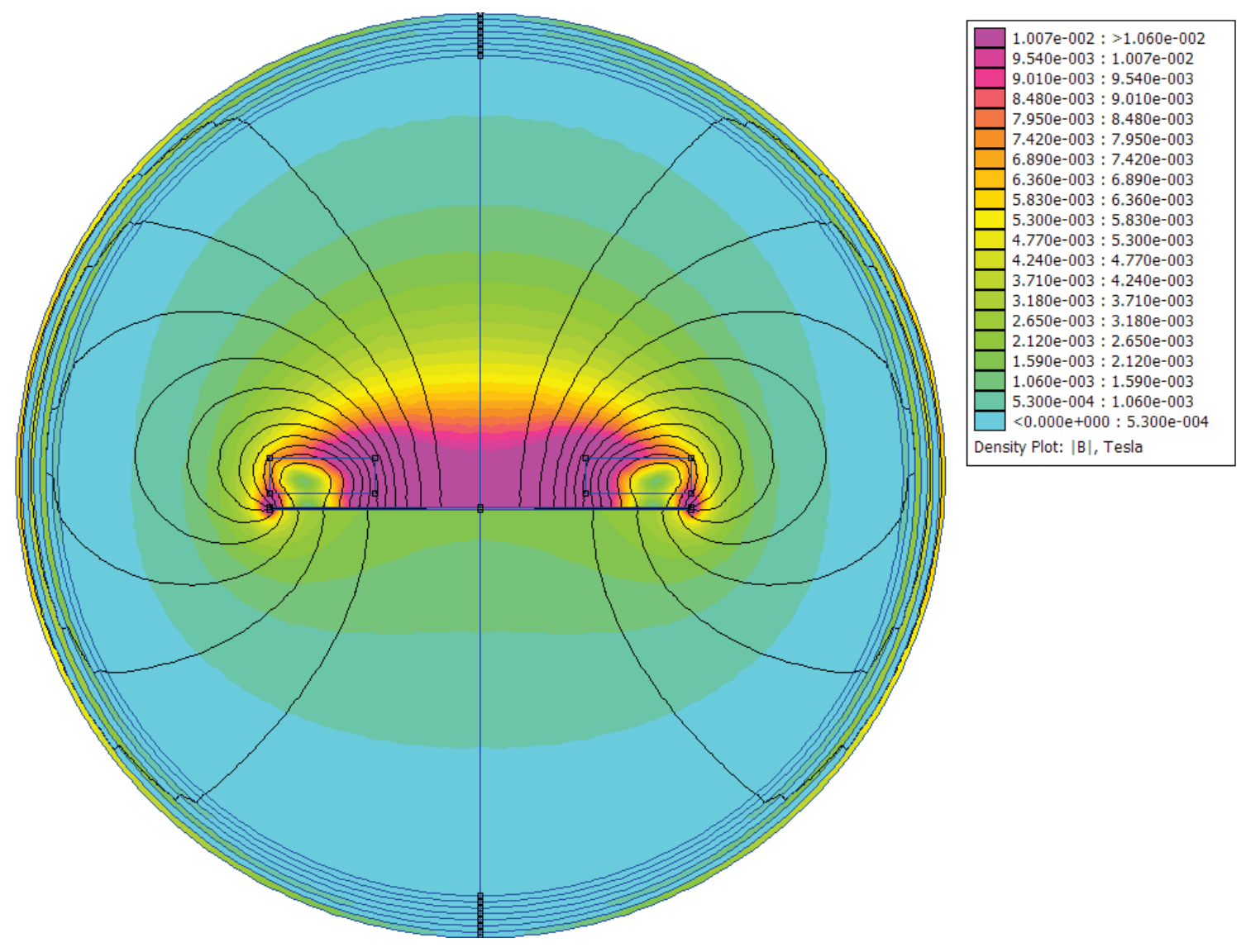

Figure 3.12 Cross-sectional view of the simulated flux density of the magnetic field generated by the transmitter coil with ferrite shield at 2 A current.

The strength of the magnetic field corresponds directly with the efficiency of the transmitter/receiver pair in space. shows the efficiency of the charging system at varying vertical offsets through animal tissue. Figure 3.13 shows the efficiency of wireless power transfer as functions of both vertical and horizontal offset from the transmitter coil. There is a significant drop in efficiency as distance between the transmitter and receiver increases (Figure 3.13b). However, there is much less attenuation in the horizontal direction (Figure $3.13 \mathrm{c})$. 
(a)

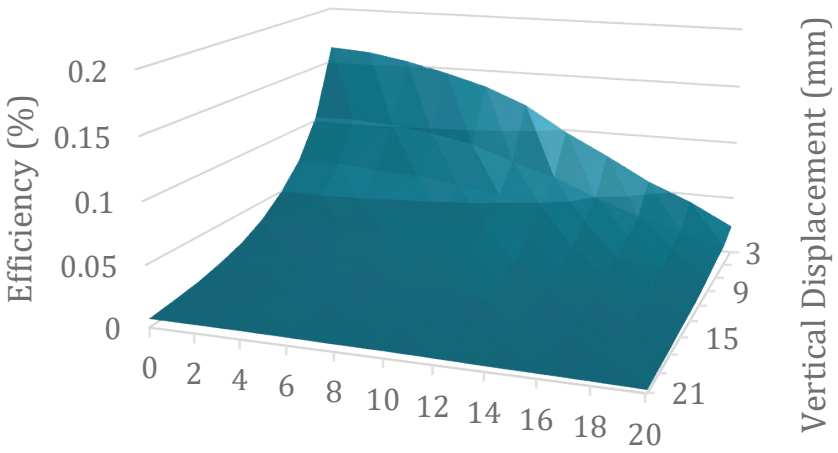

(b)

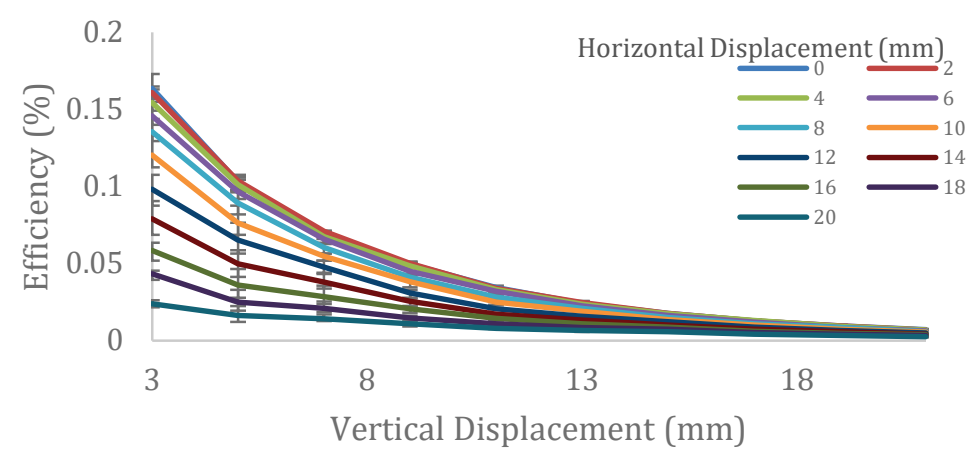

(c)

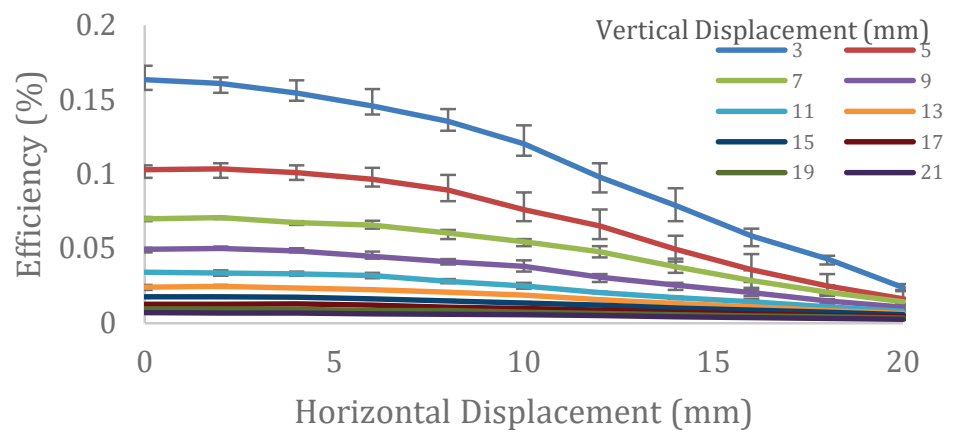

Figure 3.13 Wireless power transfer efficiency through animal tissue showing (a) both vertical and horizontal effects of distance, (b) effects of displacement in the vertical direction at varying horizontal displacements, and (c) effects of displacement in the horizontal direction at varying vertical displacements.

To improve the efficiency of the wireless power transfer system in vivo, a $0.3 \mathrm{~mm}$ thick sheet of ferrite material (Wurth Electronics Inc, Germany) is attached to both transmitter and receiver coils. 


\subsubsection{Battery Charging}

The signal received by the receiver coil is rectified by a cascade containing a full-bridge rectifier, capacitor, and zener diode (Figure 3.14). The bridge rectifier used is the CDHD2004 (Bourns Inc., Altadena, CA, USA), which features a $0.5 \mathrm{~V}$ forward drop to minimize signal attenuation. A $5.6 \mathrm{~V}$ zener diode protects the circuit from voltages that will cause component breakdown. A capacitor is used to limit the amount of voltage ripple according to the following equation:

$$
V_{\text {ripple }}=\frac{I}{C \cdot f}
$$

where $I$ is the current to the battery charger, $C$ is the size of the capacitor, and $f$ is the frequency of the rectified signal. So to obtain $100 \mathrm{~mA}$ at $2 \mathrm{kHz}$, a $100 \mathrm{uF}$ capacitor will limit the voltage ripple to around $250 \mathrm{mV}$.

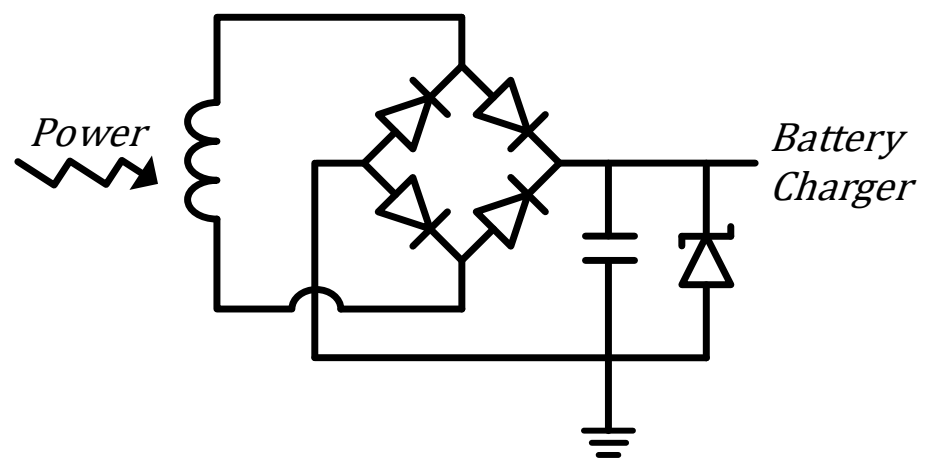

Figure 3.14 Schematic for the rectification of the received power signal. Current is induced in the inductor by a nearby magnetic field. This AC current is then rectified using a diode bridge, capacitor, and zener diode.

If the rectified signal is at least $3.75 \mathrm{~V}$, the battery charger (MCP73831, Microchip Technology Inc., Chandler, AZ, USA) is enabled. This chip provides the desired charge profile for lithium-polymer batteries (Section 3.4.3). A constant current is provided until the battery is at approximately $70 \%$ of its capacity, then the current drops and a constant voltage is provided to fill the remainder of the battery. 


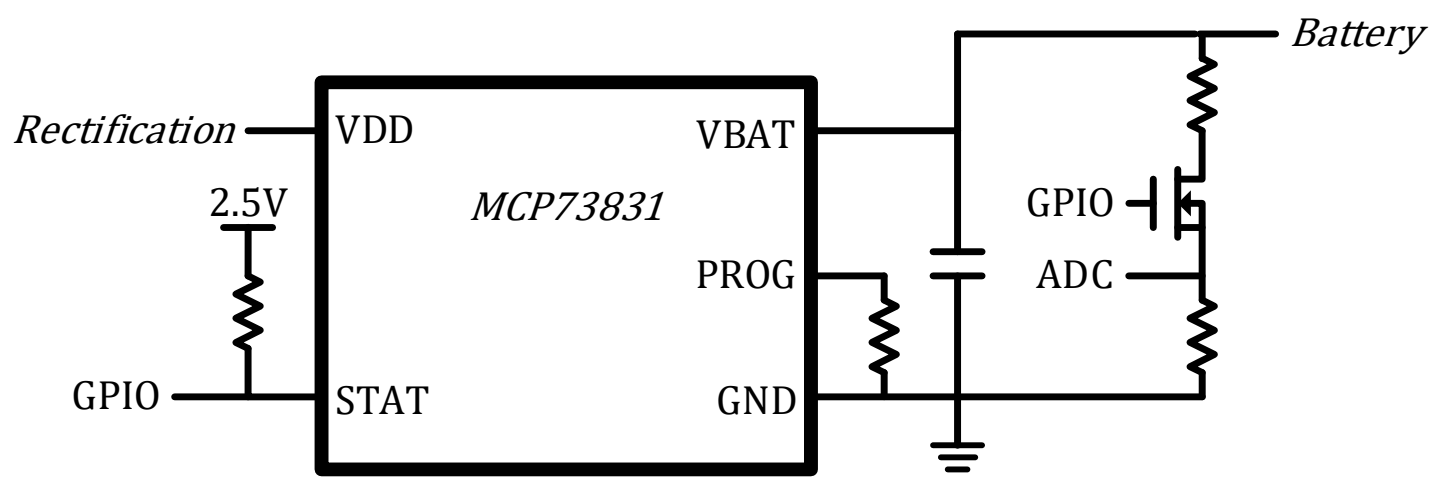

Figure 3.15 Schematic of the battery charger, charge sense, and battery level monitoring circuit.

Because the efficiency of the receiver is significantly dependent on the relative position of the transmitter and receiver coils, the current supplied to the battery changes with the position of the device relative to the transmitter (Figure 3.16). The behavior is proportional to the square root of the efficiency data. A sharp drop is observed in the vertical direction, with a more gradual decrease in current in the horizontal direction. 
(a)

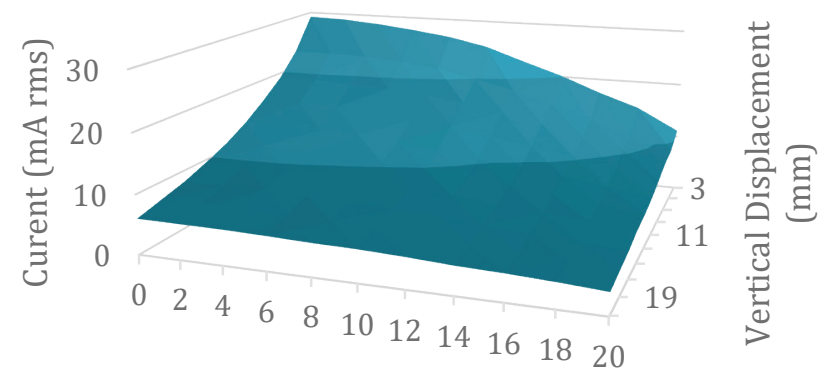

Horizontal Displacement (mm)

(b)

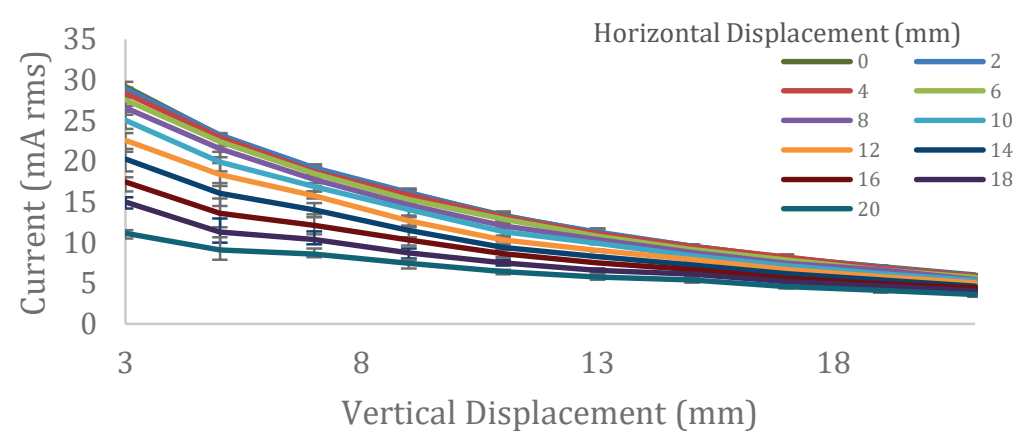

(c)

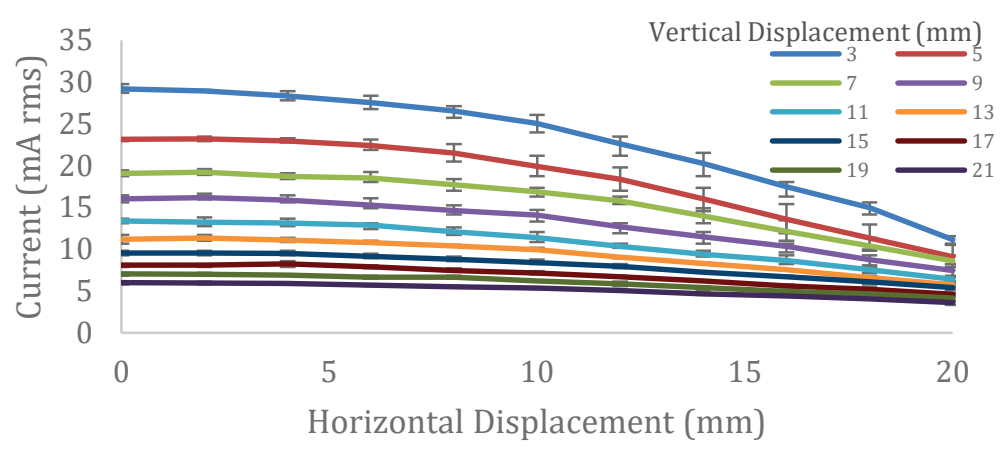

Figure 3.16 Current received through wireless power transfer efficiency through animal tissue showing (a) both vertical and horizontal effects of distance, (b) effects of displacement in the vertical direction at varying horizontal displacements, and (c) effects of displacement in the horizontal direction at varying vertical displacements.

Because of this dependence on the position of the device for charging, the charge state of the battery charger is provided by the STAT pin of the battery charger IC. When connected to a pull-up resistor, this pin will be active low when the battery is charging. This is read 
using GPIO and may be reported to the user over Bluetooth. This is used to confirm that the device is correctly positioned.

The battery voltage may also be monitored using the low-power transistor configuration shown in Figure 3.15. Current will only pass through the battery monitoring path when the transistor is turned on, which allows power to be saved when not measuring. When the transistor is enabled, the ADC reads the voltage relative to the overall voltage of the battery, which may be reported to the user using Bluetooth. The measurement profile resulting from this configuration is shown in Figure 3.17.

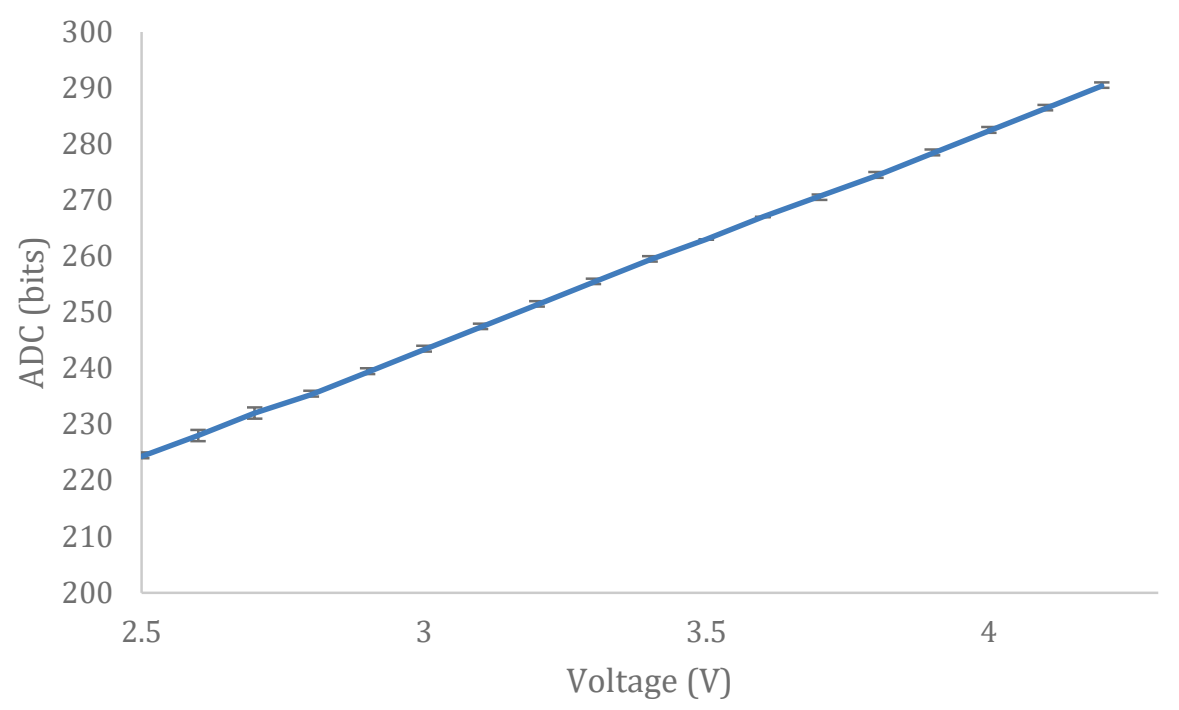

Figure 3.17 Battery voltage monitor profile $(n=3)$. The measured voltage $\left(\mathrm{V}_{\mathrm{in}} / 2\right)$ is not isolated from the $\mathrm{ADC}$, so additional resistances pull the measurement away from the expected curve. Due to consistent behavior, the voltage monitor is simply characterized instead of introducing a voltage follower to the circuit.

Two main voltage rails are used in this device. The battery provides a nominal $3.7 \mathrm{~V}$ to the piezoelectric driver circuit, but most other components can run at a lower $2.5 \mathrm{~V}$. An MCP1700 (Microchip Technology Inc., Chandler, AZ, USA) is used to provide this second voltage rail (Figure 3.18) to the microcontroller and other components that operate at the same logic level. 


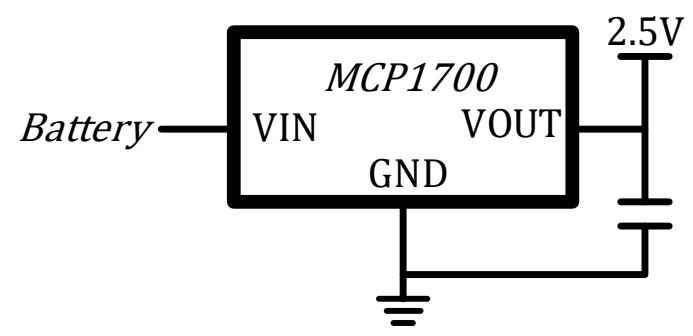

Figure 3.18 Schematic of the power supply for $2.5 \mathrm{~V}$ components.

\subsubsection{Battery}

The battery used in this design is the SparkFun DTP502535 (SparkFun Electronics, Boulder, CO, USA). This lithium polymer battery has a nominal voltage of $3.7 \mathrm{~V}$ and a nominal capacity of $400 \mathrm{mAh}$. It can provide up to 0.5 capacity $(200 \mathrm{~mA})$ of current, which is sufficient to drive the piezoelectric actuator which draws a peak current of roughly 130 mA. During constant actuation, this battery can provide current for just over an hour before the voltage drops below $3 \mathrm{~V}$.

The discharge curve representative of lithium polymer batteries such as the DTP502535 is shown in Figure 3.19. The battery may be charged up to a maximum voltage above the battery's nominal voltage. In the DTP502535, that maximum voltage is $4.2 \mathrm{~V}$. The voltage supplied by the battery decreases during use. The nominal voltage is the voltage provided by the battery for most of its lifetime. Near the end of the battery life, the voltage drops sharply. In many applications, the full capacity of the battery cannot be used before the battery is unable to provide power sufficient for the application. The battery used in this work has a nominal voltage of $3.7 \mathrm{~V}$, but is fully charged at $4.2 \mathrm{~V}$. The highest voltage required for actuation in this device is $3 \mathrm{~V}$. Roughly $97 \%$ of the battery capacity can be used before dropping below $3 \mathrm{~V}$. However, the current required by the boost converter cannot be provided by the battery at this low capacity, so the usable lifetime of the battery is actually less than this. 
(a)
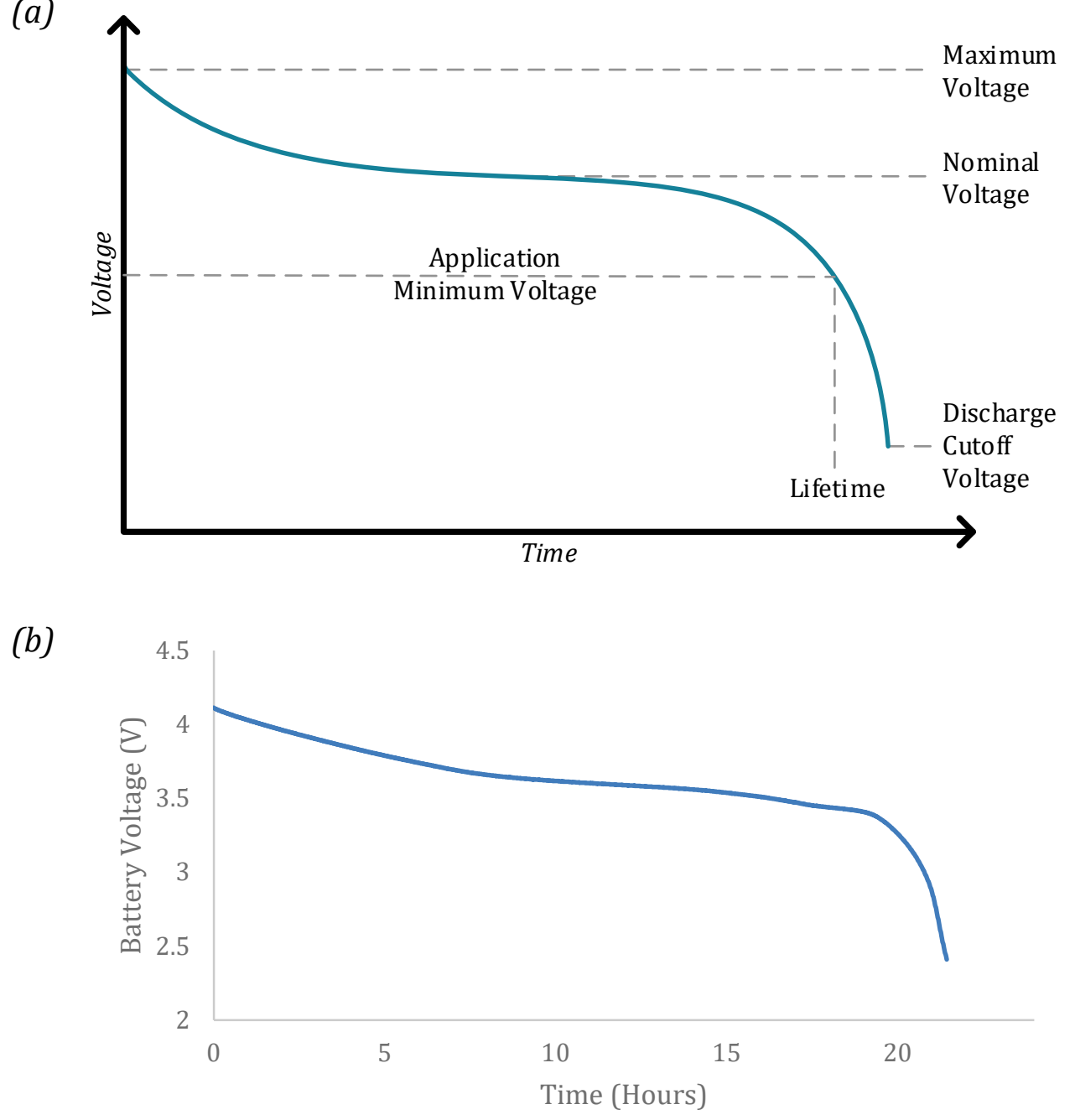

Figure 3.19 (a) Typical battery discharge profile for lithium polymer batteries. (b) Measured battery discharge curve for DTP502535.

The battery charge profile for lithium polymer batteries such as the DTP502535 is shown in Figure 3.20. Lithium polymer batteries are charged using a constant current/constant voltage $(\mathrm{CC} / \mathrm{CV})$ profile. During the first stage of charging, a constant current is supplied to the battery until it has reached roughly $70 \%$ of its total capacity. The profile then switches to constant voltage mode, where the voltage applied to the battery is roughly equal to the final capacity voltage as the current decreases logarithmically. 
(a)

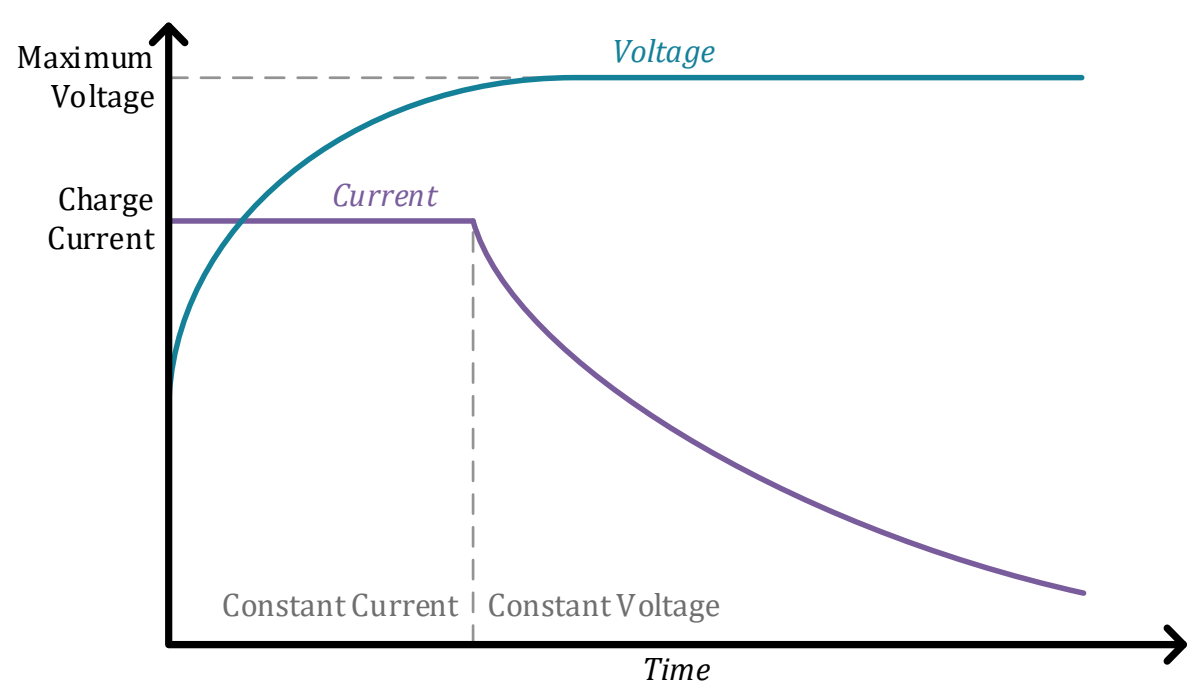

(b)

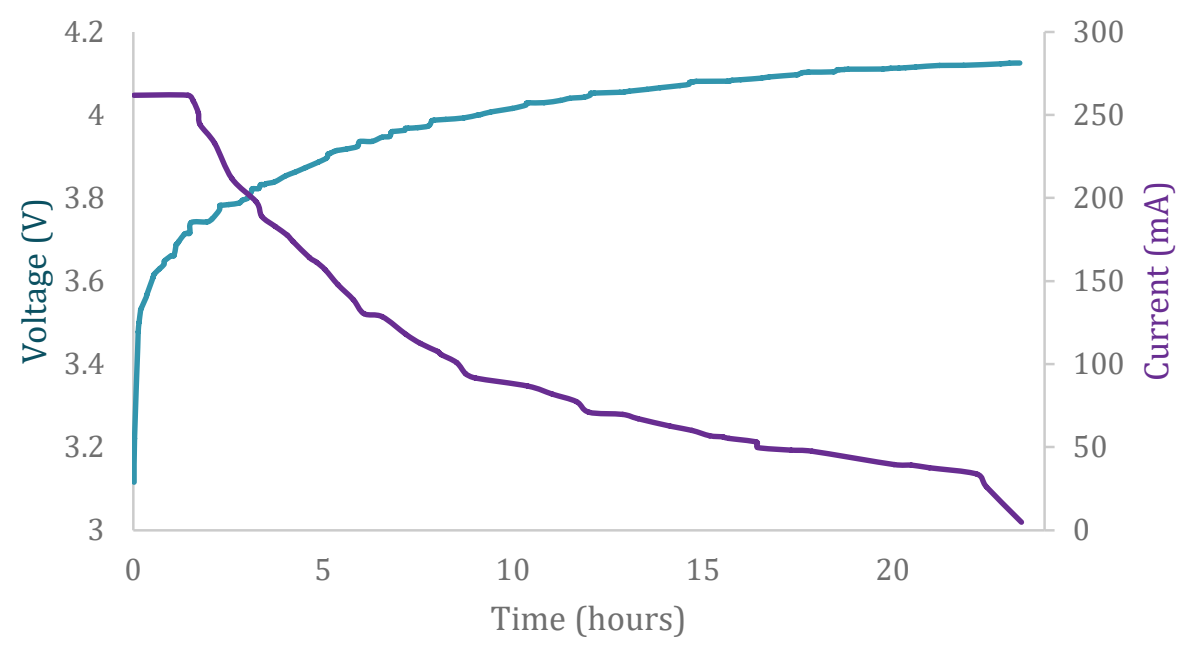

Figure 3.20 (a) Typical battery charging profile for lithium polymer batteries. (b) Battery charging curve obtained using the DTP502535.

\subsubsection{Power Budget}

Power budgets are commonly used to identify parts or subsystems that can be modified to lengthen the lifetime of a battery-powered device.

The average current and power consumption of the major subsystems of this device were measured using the National Instruments MyDAQ (Austin, TX, USA). Current and voltage 
measurements were taken at a sampling frequency of $10 \mathrm{kHz}$. Five seconds of data was collected and the time average current and voltage were taken to get the averages consumed each second. There was significant noise measured in the $1 \mu \mathrm{A}$ range, so the results shown in Table 3.2 are truncated to a $10 \mu \mathrm{A}$ precision.

Table 3.2 Experimental average current and power consumption for the major subsystems of this device.

\begin{tabular}{|c|c|c|}
\hline Component & Current $(\mathrm{mA})$ & Power $(\mathrm{mW})$ \\
\hline \multicolumn{3}{|l|}{ Microcontroller } \\
\hline Advertising & 0.02 & 0.10 \\
\hline Connected, not sensing & 0.35 & 1.43 \\
\hline Connected, sensing & 1.32 & 5.44 \\
\hline \multicolumn{3}{|l|}{ Piezo Driver } \\
\hline Idle & $<0.01$ & $<0.01$ \\
\hline Active & 22.52 & 92.94 \\
\hline \multicolumn{3}{|l|}{ Battery Monitor } \\
\hline Off & $<0.01$ & $<0.01$ \\
\hline On & $<0.01$ & $<0.01$ \\
\hline \multicolumn{3}{|l|}{ Relays } \\
\hline Off & $<0.01$ & $<0.01$ \\
\hline On & 2.69 & 6.71 \\
\hline
\end{tabular}

Most of the values collected make sense. The currents measured in the microcontroller tests are close to the values advertised in the BLE113 datasheet. The use of a MOSFET in the battery monitor subsystem should draw negligible current when off and the large resistors keep the current consumption in the on phase in the sub-microvolt range. The relays drew slightly less current than expected in its on stage. The piezoelectric driver current is significantly lower than what is expected. However, the use of the piezoelectric driver 
drains the $400 \mathrm{mAh}$ battery in just over one hour, which requires currents significantly higher than those measured in this test.

This measurement error may be due to multiple factors. First, this measured value may be too low, and may require more accurate sampling or a faster sampling rate to achieve more accurate measurements. Second, a battery is not able to provide its full current capacity in most applications. Third, the capacity is not constant at all currents. At higher current draw the lifetime of the battery relative to its capacity decreases. This is especially true in the piezoelectric driving circuit which draws a peak current of nearly $200 \mathrm{~mA}$ ( 0.5 capacity). The same behavior is seen when the device is in sensing mode, where it can last for 72.7 hours before dying - slightly longer that the 59.7 hours predicted by the power budget. Finally, due to the non-linear behavior of batteries, a theoretical power budget does not necessarily reflect the lifetime of the device [85]. Power budgets are effective tools at identifying components or subsystems that are drawing too much power but are less reliable when predicting the lifetime of the device.

\subsubsection{Thermal Considerations}

When using electromagnetic coils for wireless power transfer, it is important to consider the heat introduced to the body and the effect this heat has on the cells. The field of radiology contains a significant volume of research regarding temperature thresholds. One method of calculating safe temperature thresholds is using the cumulative equivalent minutes at $43^{\circ} \mathrm{C}\left(\mathrm{CEM} 43^{\circ} \mathrm{C}\right)$ [86]. Using this method, temperature $T_{i}$ and time exposed to that temperature $t_{i}$ during a time interval $i$ are used to calculate the equivalent minutes at $43^{\circ} \mathrm{C}$ using the equation:

$$
C E M 43^{\circ} \mathrm{C}=\sum_{i=1}^{n} t_{i} \cdot R^{43-T_{i}}
$$

where $R=0.25$ for temperatures less than $43^{\circ} \mathrm{C}$ and $R=0.5$ for temperatures greater than $43^{\circ} \mathrm{C}$. Based on a review by Van Rhoon et al. [87], a CEM $43^{\circ} \mathrm{C}$ of 9 minutes is appropriate in most cases. This value is supported by $\mathrm{CEM} 43^{\circ} \mathrm{C}$ data regarding earliest damage to 
internal organs such as the kidney and spleen occurring around this value in rats. Based on the above equation and given a roughly 8-hour charge time, the safe temperature threshold is approximately $40^{\circ} \mathrm{C}$.

At $1 \mathrm{kHz}$, the transmitter coil dissipates power equal to:

$$
P=I_{R M S}{ }^{2} * R
$$

In this system, an external transmitter coil is located in the animal cage below the tube that rats commonly sleep in. In a wireless battery charging system, there are three components to consider for thermal dissipation: the transmitter coil, the receiver coil, and the battery itself. In this loosely-coupled system, the transmitter coil outside the animal's body is the greatest concern due to the presence of significantly higher currents. Figure 3.21 shows the maximum temperature reached by the external coil when continuously active in a room at roughly $25^{\circ} \mathrm{C}$ with minimal air circulation and with a cooling fan beneath the coil drawing heat from it.

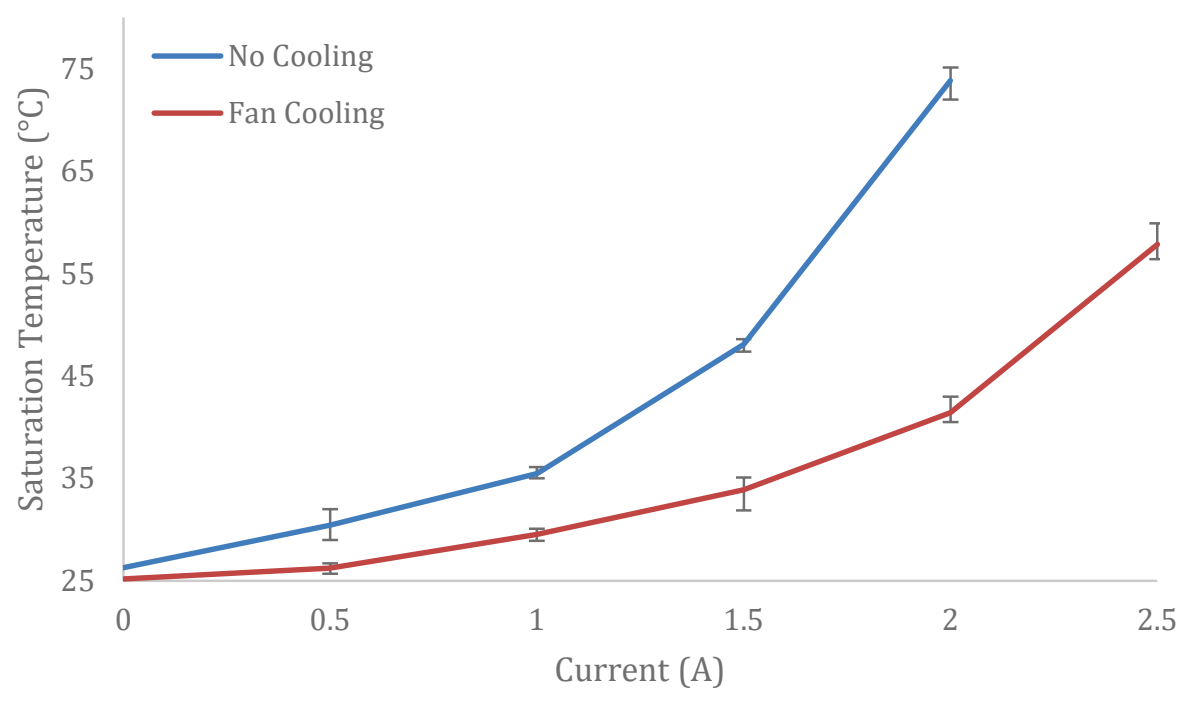

Figure 3.21 Maximum temperatures reached by the external transmitter coil $(n=3)$ as a function of supply current (RMS). 
The temperature of the transmitter and receiver can be monitored in order to keep the temperature within a desired range. The power supply can be temporarily disabled if the temperature increases too significantly.

\subsection{Software}

\subsubsection{Implant Software}

On startup, the telemetry unit begins to advertise information such as its name and appearance once per second until a connection is initiated by another device. All peripheral components remain off until they are enabled by an external device. Both sensing and actuation modes are disabled, so all relays are off.

When a connection has been established, a polling timer is started to read the charge state of the battery and the battery voltage. The charge state is polled every second the help the user to position the animal on the charger. The battery voltage is updated every 30 seconds. These may both be read at any time by the client device.

The implant is controlled by wirelessly updating Bluetooth attributes. Three modes are available. In off mode, sensing and actuation pathways and their related timers and other peripherals are off. When the device is put in actuation mode, the relays that complete the actuation pathway are enabled. Control of the piezoelectric driver, which is performed using I2C communication, is accessible to the user through another attribute. This attribute allows easy access to the default actuation profile ( $40 \mathrm{~V}$ at $35 \mathrm{~Hz}$ for 20 minutes) but may be altered to enable other actuation profiles.

In sensing mode, the relays that complete the sensing pathway are enabled and a polling timer is started to read data at $30 \mathrm{~Hz} .10$ data points are collected and packetized before being transmitted at once to reduce transmitter power consumption. 


\subsubsection{PC Software}

PC software was written to manage communication and data processing using the Windows Presentation Foundation (WPF) graphical framework in C\#. This software is designed to interface with the BLED112 wireless USB dongle (Silicon Labs, Austin, TX, USA). It manages discovered and connected devices, allows the user to control actuation and sensing of the target telemetry unit, and receives, processes, and saves receives data to CSV files.

On startup, the software does not have access to Bluetooth communication. To enable this communication, the communication port corresponding to the BLED112 must be selected and serial communication must be opened Figure 3.22.

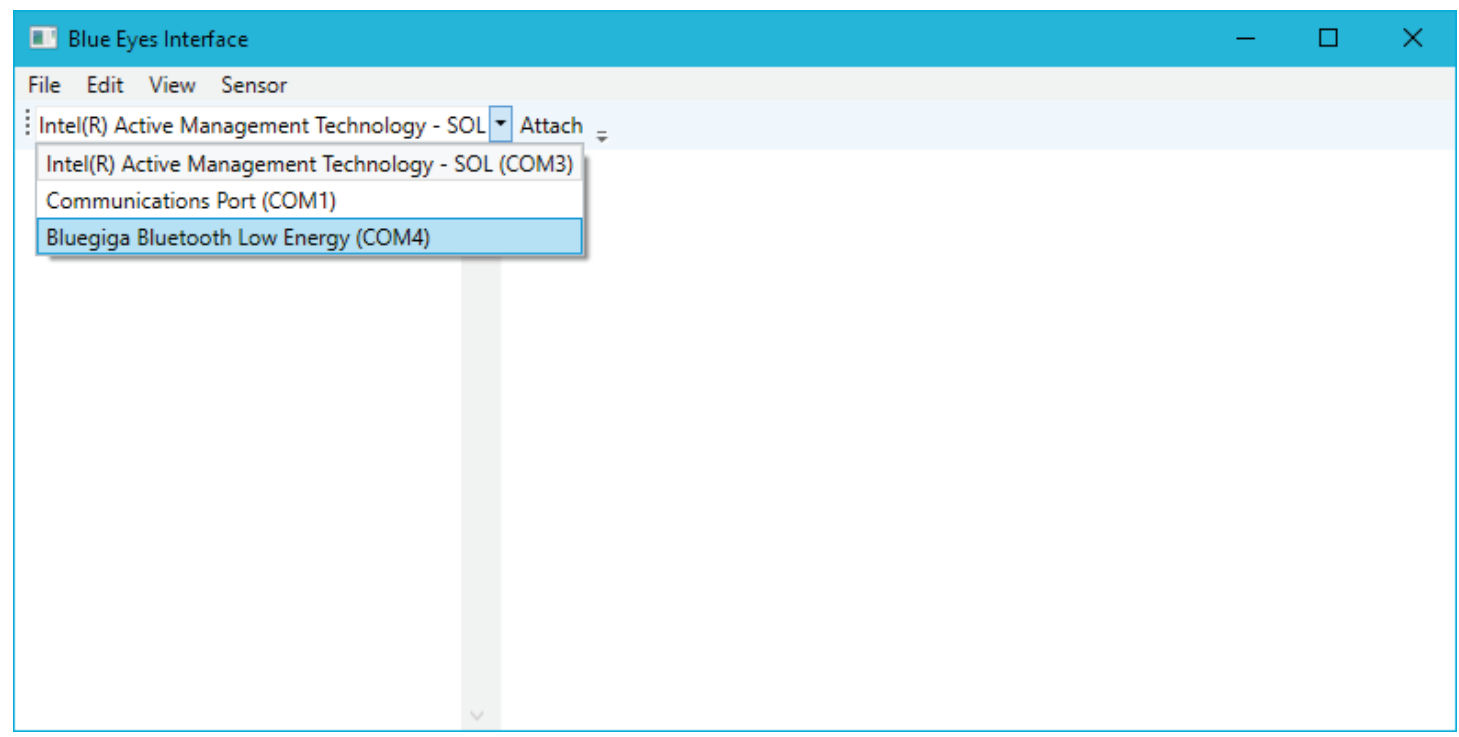

Figure 3.22 Port selection box. This is used to open serial communication with the BLED112 to enable Bluetooth communication.

Once the BLED112 has been connected, a list of nearby discovered Bluetooth devices showing the name and address appears on the left side of the window (Figure 3.23). Each entry is expandable to reveal the signal strength, bond number, and all data that the device has advertised. A command to initiate a connection with a desired device may be performed using the connect buttons. 


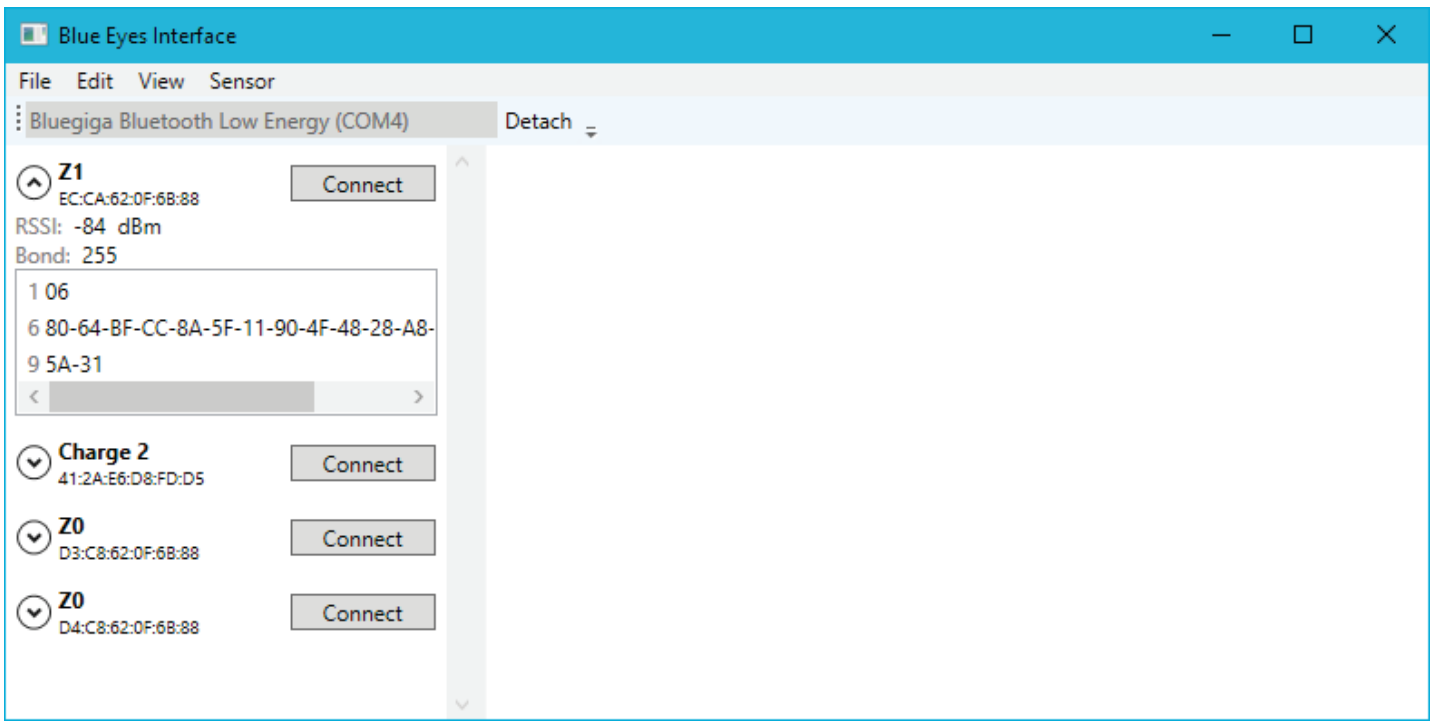

Figure 3.23 List of discovered devices. The entry for Z1 has been expanded to reveal additional information including signal strength, bond, and a list of advertised information in hexadecimal format.

One or more connected devices appear as tabs on the right side of the interface (Figure 3.24). In each tab is a data table containing the full list of generic attributes available in that device. As the value of each of these attributes is made known to the client, the table is updated. 


\begin{tabular}{|c|c|c|c|c|c|c|c|}
\hline 피 Eyes Interfa & & & & & - & $\square$ & $x$ \\
\hline File Edit View & & & & & & & \\
\hline Bluegiga Bluetooth & gy (COM4) & & etach & & & & \\
\hline (A) $\mathrm{Z1}$ & Disconnect & $\mathrm{Z1}$ & & & & & \\
\hline $\begin{array}{l}\text { EC:CA:62:0F:6B:88 } \\
\text { RSSI: }-87 \mathrm{dBm}\end{array}$ & & & Description & Value & & & \\
\hline Bond: 255 & & 28 & CharacteristicUserDescription & & & & $\wedge$ \\
\hline 106 & & 29 & PrimaryService & & & & \\
\hline $680-64-B F-C C-8 A-$ & $4 F-48-28-A 8-$ & 30 & Characteristic & & & & \\
\hline $95 \mathrm{~A}-31$ & & 31 & ChargeState & 01 & & & \\
\hline & $>$ & & ClientCharacteristicConfiguration & & & & \\
\hline ( $)$ Charge 2 & Connect & 33 & Characteristic & & & & \\
\hline 41:2A:E6:D8:FD:D5 & & 34 & BatteryVoltage & $0 \mathrm{E}-01$ & & & \\
\hline ( () $Z_{03 \cdot C 8 \cdot 62 \cdot 05 \cdot 6 B-88}^{Z}$ & Connect & 35 & ClientCharacteristicConfiguration & & & & \\
\hline & & 36 & Characteristic & & & & \\
\hline ( $)$ Z0 & Connect & 37 & Temperature & & & & \\
\hline & & & ClientCharacteristicConfiguration & & & & \\
\hline & & & CharacteristicUserDescription & & & & $\checkmark$ \\
\hline
\end{tabular}

Figure 3.24 Table showing connected devices and the most recent data that has been received from them.

Values may be updated by right-clicking on the attribute to change and selecting "Edit..." from the context menu. This will open a separate window that will allow the input of a decimal value that may be written to the server. If the attribute is read-only or an invalid number is entered, this will fail.

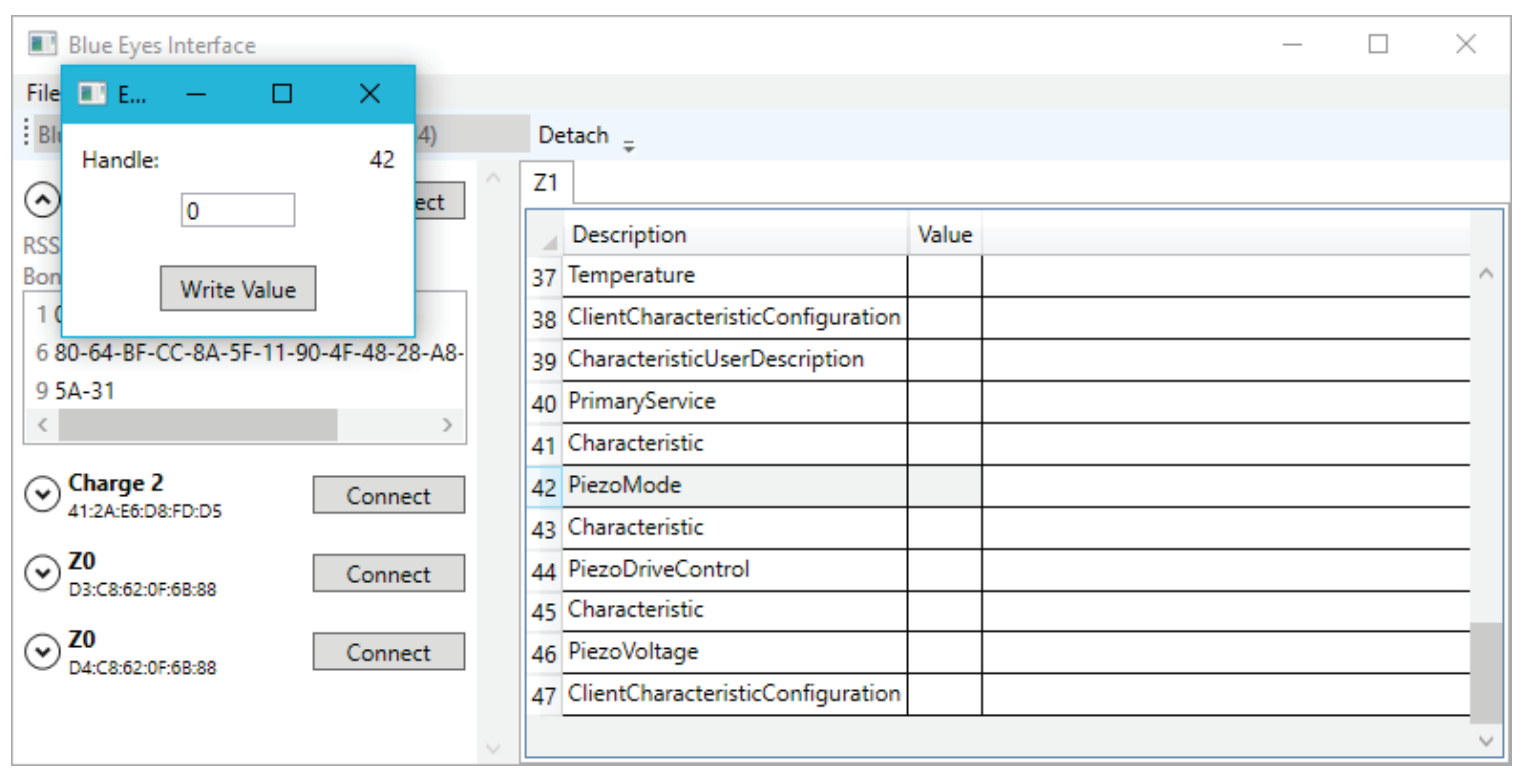

Figure 3.25 Window allowing writing of values to the server. 
As data is received from the implanted device, it is logged to a local CSV file. This data may be processed to extract the magnitude of the loads applied and other information provided by the sensor.

At time of submission (August 12, 2019), the code for this software is available at https://github.com/bradleydavidnelson/BlueEyes.

\subsection{Enclosure}

The three-piece enclosure (Figure 3.26) for this telemetry unit was 3D printed from FormLabs Dental LT resin (RS-F2-DLCL-01) using the FormLabs Form 2 printer. The superficial face of the enclosure features a raised boss. The inductive coil is assembled around this boss and glued into place. Two eyelets on the exposed side allow the telemetry unit to be sutured to the abdominal wall. A small walled section surrounds the coil and allows less sharp edges on the exposed animal.

The telemetry packaging described in this work uses the same materials and methods for the enclosure and wires to the fixation plate as those used in previous implants used in this animal model $[29,81]$, except the 3D printed packaging is of a different size. While 3D printed packaging and the polymer coating are limited to short-term implants $(<29$ days [88]), it is commonly used in animal studies like this work [29, 81]. 

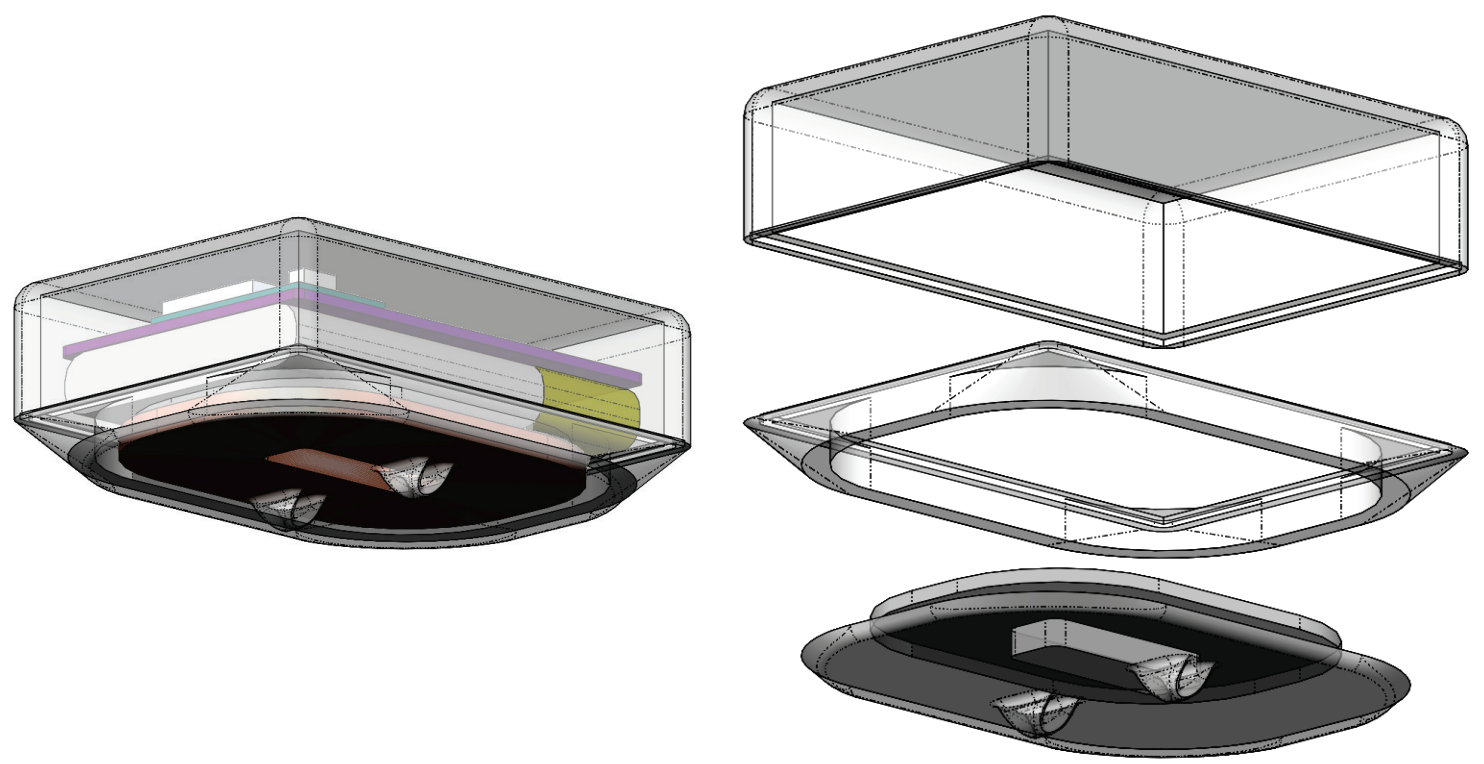

(c)

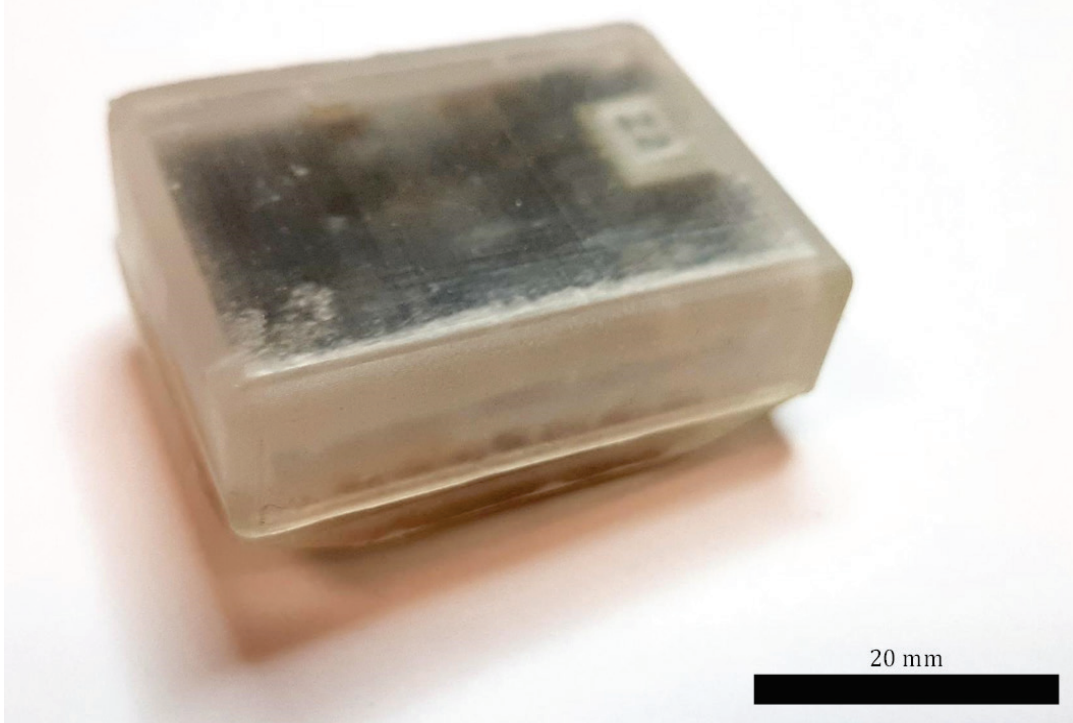

Figure 3.26 3D printed enclosure (a) containing the device components, (b) in exploded view, and (c) as photograph. The bottom is the superficial side of the telemetry unit.

Two 5-inch-long PFA-coated stainless-steel wires (A-M Systems, Sequim, WA, USA) enclosed in a silicone sleeve (A-M Systems, Sequim, WA, USA) connect the telemetry 
unit to the fixation plate. These wires exit the telemetry unit from the side and are glued inside the enclosure to reduce the risk of being pulled loose.

The packaging is assembled in multiple stages. The WPT coil is wound around the superficial component of the packaging. A sacrificial winding post is printed on this section to assist in more uniform coil winding using a coil winding machine. This post is then removed, and the wall is glued into place. The battery, WPT coil, and fixation plate are soldered to the PCB and the assembly is closed. A layer of Dymax 1072-m (Dymax Corporation, Torrington, CT, USA) is applied to the entire surface of the telemetry packaging and cured using UV light.

\subsection{Electromagnetic Signal Propagation in Tissue}

To demonstrate the efficacy of the electromagnetic systems used in this telemetry platform, namely the Bluetooth communication and wireless power transfer systems, fully assembled systems containing both the telemetry unit and the fixator $(n=3)$ were implanted into adult female Sprague Dawley (SD) rat (Charles River, Wilmington, MA, USA) cadavers. The animals and surgical procedures follow a relevant animal model [48]. While there are differences in the dielectric properties of live and dead tissue [89], the use of a cadaver model provides a similar environment to the body without requiring the use of live animals for preliminary verification.

The surgical areas were prepared by shaving the abdomen and target hind limb of the animal. A medial incision was then made across the ventral abdomen through the skin and superficial musculature to provide access to the abdominal cavity. An anterolateral skin incision was also made across the target hind limb to expose the underlying muscle. The hind limb fascia was separated using blunt dissection to expose the quadriceps muscles, and a keyhole incision was made in the abdominal wall between the abdominal cavity and the target hind limb. The fixation plate was inserted from the abdominal side of this incision into the exposed femoral region so that the wire connecting the fixator and telemetry passed through the keyhole incision. The telemetry unit was gently inserted into the abdominal 
cavity between the abdominal organs and subcutaneous muscles. The telemetry unit was anchored to the abdominal wall by suturing the eyelets through the muscle and skin. Both the abdominal muscle and abdominal skin were then sutured shut.

Blunt dissection was used to separate the exposed quadriceps muscles to expose the femur from the femoral head to the lateral condyle. The fixation plate was placed on the femur and clamped using a stainless-steel c-clamp. A stainless-steel clamping plate was used to distribute the load from the clamp across the fixation plate to reduce the risk of damage. With the fixation plate held in place by the clamp, four holes were drilled through the femur via the screw holes in the riser plates. Stainless steel screws were inserted into the drilled holes to hold the fixation plate to the bone. Once the two outermost screws were in place, the clamp and clamping plate were removed to allow access to the innermost screw-holes. The attachment of the fixator to an explanted femur is shown in Figure 3.27, with a $6 \mathrm{~mm}$ osteotomy shown for scale.

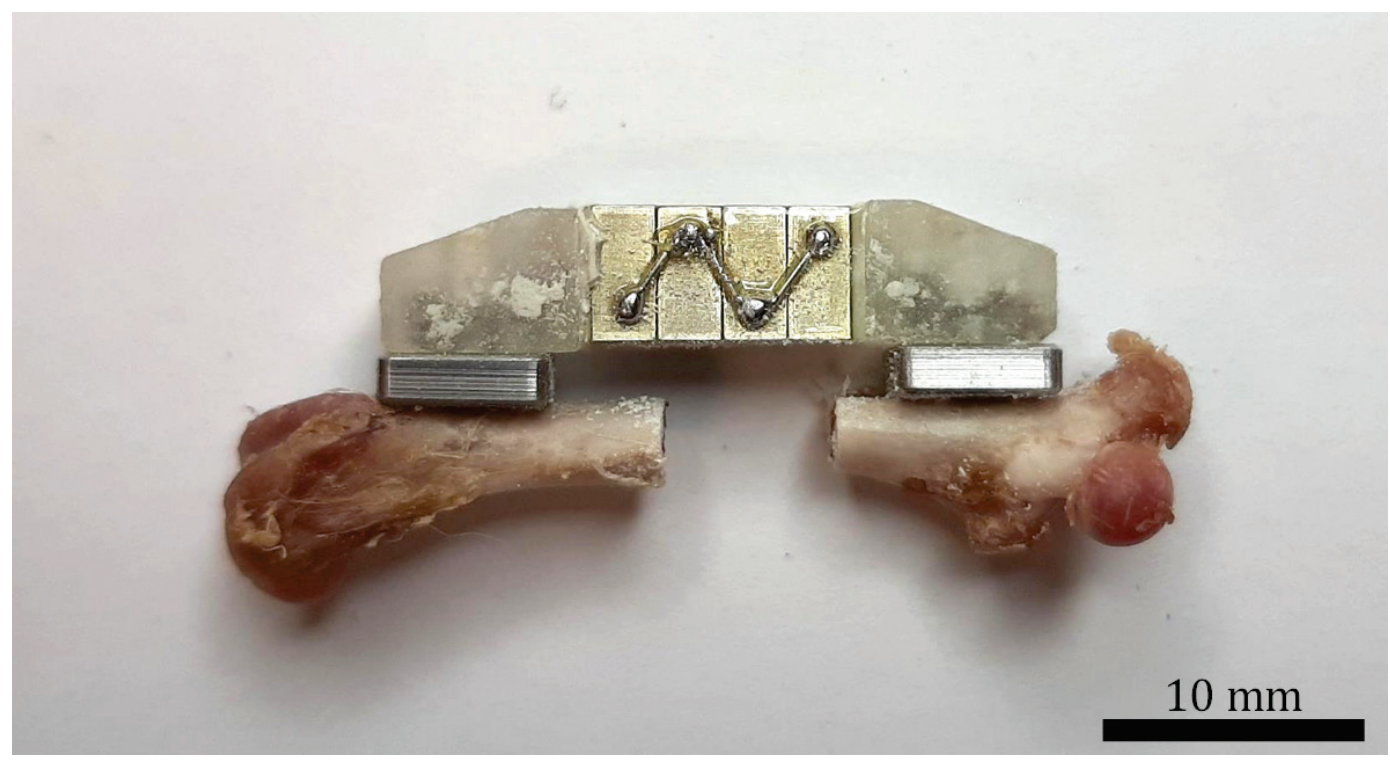

Figure 3.27 Fixator attached to explanted rat femur via screws. The defect size is 6 $\mathrm{mm}$.

Once the plate was firmly attached to the femur via the four screws, a 3D printed surgical guide was placed around the fixation plate. This guide has notches separated by $6 \mathrm{~mm}$ for reproducible critical-sized osteotomy. The guide also serves to protect the plate from 
damage during the osteotomy process. Gigli wire was used to create the $6 \mathrm{~mm}$ defect through the femur by cutting the bone both the proximal and distal notches in the surgical guide. The resulting bone defect was removed. After the defect was made, the muscle was sutured together over the fixation plate and the skin was sutured shut.

After surgery, the PC software described in section 3.5.2 was used to establish a connection with the telemetry unit. The telemetry unit was switched to load monitoring mode and the fixator was loaded using manual cyclic compression of the femur through the skin. Load monitoring mode was then disabled.

An example of the data collected during manual cyclic loading is shown in . The loading increased from an amplitude of roughly $0.1 \mathrm{~V}$ to an amplitude of roughly $0.3 \mathrm{~V}$. Based on a loading sensitivity of the plate without any callus formation of approximately $25 \mathrm{mV} / \mathrm{N}$, this corresponds to loading of 4 to $12 \mathrm{~N}$, which is within the range of what would be expected during ambulation [90].

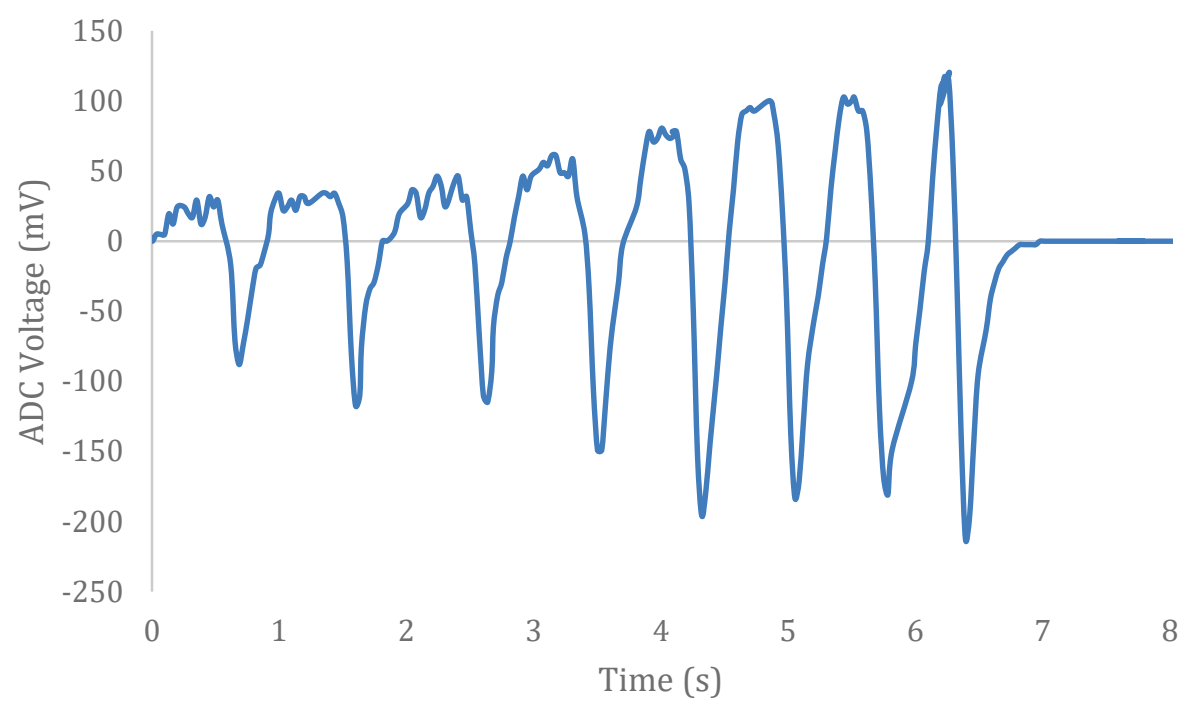

Figure 3.28 Load monitored by the fixation plate in the animal cadaver during manual actuation of the femur. The forces measured are in the range of 4 to $12 \mathrm{~N}$.

The animal was then placed on a wireless charging pad. A $1 \mathrm{kHz}$ signal was provided to the charger via the Tapco Juice J-1400 amplifier at increasing current until the battery 
charging state was wirelessly indicated in the software. The minimum current through the transmitter coil to cause the battery charger to recognize a charge was between 2 and 2.2 $\mathrm{A}_{\mathrm{rms}}$ for all three implants.

\subsection{Conclusion}

The circuitry in this section is sufficient to allow the use of the piezoelectric fixation plate in the animal model proposed in [48]. The size of the packaging is $41.8 \times 32.0 \times 15.6 \mathrm{~mm}$ which allows it to be implanted into the rat abdominal cavity. It uses BLE communication, which has sufficient range and reliability to communicate through the animal tissue to a computer within roughly $20 \mathrm{~m}$ of the animal. This allows for a wide range of testing environments in small- and medium-sized rooms. The battery used in this work can provide constant actuation for over an hour, which is more than sufficient for LMHF treatments which usually last for 15 to 20 minutes [55]. This battery may also be wirelessly recharged through the abdominal tissue so that the device may last for the duration of an $8+$ week study.

The telemetry unit described in this work is able to communicate within a $20 \mathrm{~m}$ room with minor data packet loss. To prevent loss of data, cyclic redundancy checks (CRC) are used in Bluetooth communication. These allow the receiver to evaluate whether or not the received packet contains the same data that was sent by the transceiver. If an error is detected from an advertising packet, the packet is likely to be discarded. Connected devices, on the other hand, have multiple ways to verify and request data when an error occurs. Data packets may either be initiated by the client or the server. When the client sends a read request to the server, the $\mathrm{CRC}$ can be compared with the expected value. If the CRC does not match, the client may simply repeat the read request until it receives the

correct packet. There are two methods used by BLE when data transmission is initiated by the server: notification and indications. Notifications send data but require no confirmation by the client. If the $\mathrm{CRC}$ does not match, the client may send a read request to get the 
correct data. Indications require confirmation from the client when data is sent, and the data will be sent repeatedly until an indicate packet is sent.

In this work, strain measurement data is set up to require indications. Whenever a new packet of measurement data is ready to be sent, it will continue to send that packet until it receives an indicate packet. This helps promote data accuracy for higher-frequency data. Packets of ten data points are sent every $3 \mathrm{~Hz}$, which leaves sufficient space for repeats in the case of received errors. Less vital data, such as the battery voltage, is transmitted using notifications. Error packets will be ignored, and the device will simply wait for the next voltage update. As a result, two-way communication may occur reliably even through biological tissues.

The minimum current to initiate battery charging will provide temperatures slightly above what is recommended using a CEM $43^{\circ} \mathrm{C}$ of 9 minutes. Therefore, it is desirable to not provide continuous battery charging. A pulsed charging profile will further improve cooling by allowing the coil to cool and prevent it from reaching the peak operating temperatures described in section 3.4 .5 since it takes well over an hour to reach these saturation temperatures. 


\section{Conclusions and Future Directions}

\subsection{Conclusions}

This work provides the following scientific contributions:

(1) The development of a piezoelectric fixation plate that provides localized actuation to facilitate fracture healing and local load monitoring to longitudinally observe the progress of fracture healing.

(2) The development of an implantable wireless telemetry unit for control of the fixation plate.

The piezoelectric fixator in this work is designed as a tool to allow for localized LMHF actuation and evaluate its efficacy in the healing of critical-size fractures. Current LMHF treatments rely on whole-body vibration that do not specifically target the fracture site or on localized actuation using external fixation plates. The fixation plate described in this work is (to the author's knowledge) the first internal fixation device that provides LMHF actuation to promote endochondral ossification as well as load monitoring to observe changes in the fracture stiffness throughout the healing process.

The actuation performance of the fixator allows for actuation up to approximately $20 \mu \mathrm{m}$ at the rated voltage limits of $\pm 75 \mathrm{~V}$ before bridging occurs. Furthermore, while the testing in this work was limited to the $30 \mathrm{~Hz}$ range (which appears to be optimal in the available literature), piezoelectric actuators can actuate at significantly higher frequencies. This allows for the investigation of a wide range of loading patterns with a single device.

The load monitoring performance of the fixator allows for the fixator to estimate the stiffness of the fracture callus. As the stiffness of the off-axis bone increases, the sensitivity in terms of $\mathrm{V} / \mathrm{N}$ decreases as the strain due to loading decreases.

The telemetry unit described in this work allows the piezoelectric fixation plate to be completely implantable. It uses Bluetooth Low Energy (BLE) communication to allow for 
the device to be remotely controlled by the user as well as for data collection during load monitoring. A local battery, along with boost converter and waveform shaper, control the actuation profile of the fixator and an array of solid-state relays allow the fixator to switch between actuation and sensing modes. A wireless charging system allows the battery to be recharged during implantation to allow the device to last for the duration of a several-month study.

The complete system was implanted into the cadavers of a relevant animal model to demonstrate that the system worked in vitro. Mechanical load monitoring performed as expected, and the battery was able to be recharged through the abdominal tissue of the rat.

\subsection{Future Directions}

The most prominent future direction of this work is to use the described system in a live animal study to quantify the effects of LMHF actuation on healing outcomes such as speed of healing, resulting bone strength, and resulting angiogenesis.

Other limitations and failure modes of this device may direct the future direction of development. One such failure mode is the small screws that attach the $3 \mathrm{D}$ printed endcaps to the stainless-steel riser plates used in this design. These screws have a tendency to shear, leaving the fixation plate disconnected from the femur. This may be resolved by machining the endcaps out of a single material. If the material is sufficiently hard, the ceramic component between the piezoelectric and the endcaps may be removed to reduce the size of the fixator. This design may be seen in Figure 4.1. 


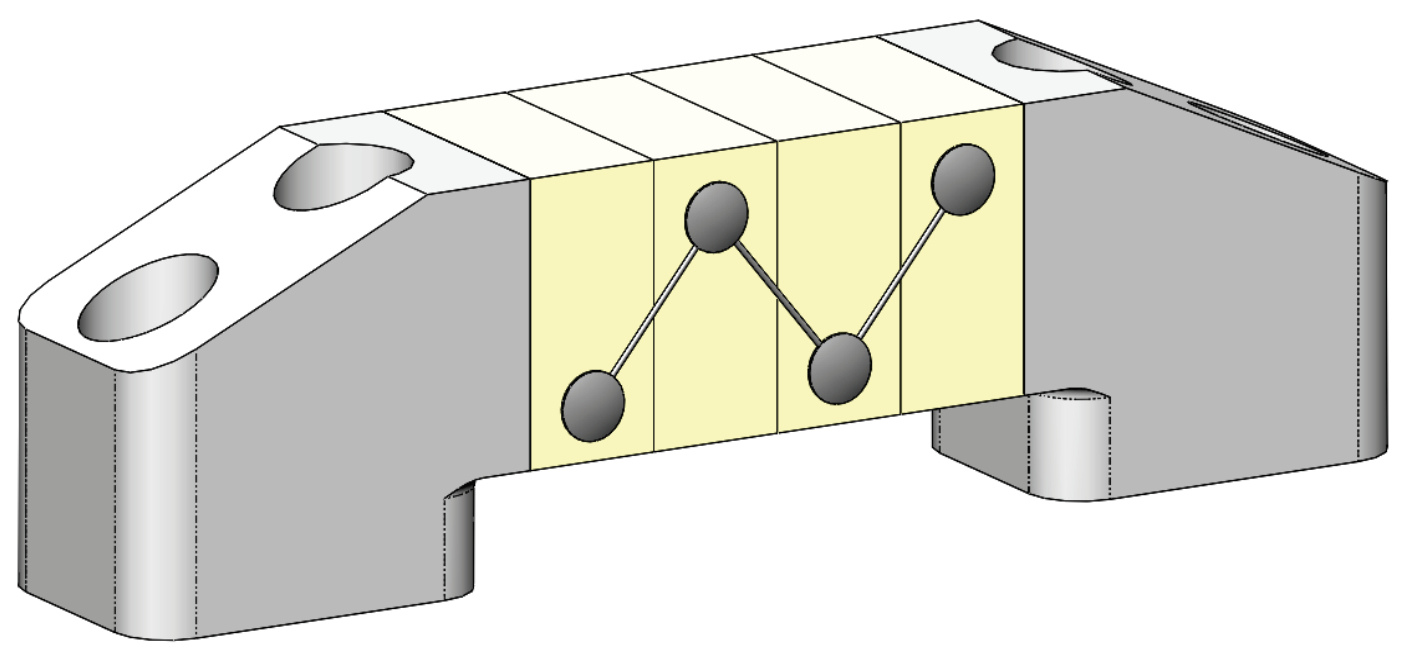

Figure 4.1 Potential future fixation plate design featuring machined endcaps. The removal of separate riser plates eliminates a common failure mode experienced in similar implants.

Another limitation in the current design is the wireless battery charging system. While the loosely-coupled system described in this work is effective it is inefficient, results in significant heat generation, and takes up more space in the telemetry unit than a more tightly coupled system with fewer turns. The current system has the benefit of greater freedom of movement in both horizontal and vertical directions, but digital tuning of the coils may be used to improve resonant tuning systems. Further work is required to improve the efficiency of the wireless power system to reduce the generated heat.

The packaging of the telemetry unit may also be improved. 3D printed packaging is useful for prototyping, but it does not provide the protection against moisture that more hermetically sealed packaging can. Environmental resilience may also be improved through parylene coating of the electronic components or the telemetry system as a whole.

While piezoelectric drift has not been an issue in this work, it is possible that the drift may be an issue in the future so that the ADC cannot capture the load monitoring signals. If this is the case, then additional circuitry such as a charge amplifier may be added to the sensing 
pathway to reduce its effects. Alternatively, a high-pass filter may be used to remove the low frequency drift. 


\section{Reference List}

[1] A. N. Pollak and S. Watkins-Castillo, I., "Injuries," in The Burden of Musculoskeletal Diseases in the United States, 3rd ed, 2014.

[2] L. Claes, S. Recknagel, and A. Ignatius, "Fracture healing under healthy and inflammatory conditions," Nature Reviews Rheumatology, vol. 8, no. 3, pp. 133143, 2012.

[3] R. Zura et al., "Epidemiology of fracture nonunion in 18 human bones," JAMA Surgery, vol. 151, no. 11, p. e162775, 2016.

[4] C. Tzioupis and P. V. Giannoudis, "Prevalence of long-bone non-unions," Injury, vol. 38, pp. S3-S9, 2007.

[5] M. Gaston and A. Simpson, "Inhibition of fracture healing," The Journal of Bone and Joint Surgery. British Volume, vol. 89, no. 12, pp. 1553-1560, 2007.

[6] D. R. Marsh, S. Shah, J. Elliott, and N. Kurdy, "The Ilizarov Method in Nonunion, Malunion and Infection of Fractures," Journal of Bone \& Joint Surgery, British Volume, vol. 79-B, no. 2, pp. 273-279, 1997.

[7] E. Antonova, T. K. Le, R. Burge, and J. Mershon, "Tibia shaft fractures: costly burden of nonunions," BMC Musculoskeletal Disorders, vol. 14, no. 1, 2013, Art. no. 42 .

[8] S. Patil and R. Montgomery, "Management of complex tibial and femoral nonunion using the Ilizarov technique, and its cost implications," The Journal of Bone and Joint Surgery. British Volume, vol. 88, no. 7, pp. 928-932, 2006.

[9] A. Phillips, "Overview of the fracture healing cascade," Injury, vol. 36, no. 3, pp. S5-S7, 2005. 
[10] H. M. Frost, "The biology of fracture healing: An overview for clinicians. Part I," Clinical Orthopaedics and Related Research, no. 248, pp. 283-293, 1989.

[11] C. Sfeir, L. Ho, B. A. Doll, K. Azari, and J. O. Hollinger, "Fracture Repair," in Bone Regeneration and Repair: Biology and Clinical Applications, J. R. Lieberman and G. E. Friedlaender, Eds. Totowa, NJ: Humana Press, 2005, pp. 21-44.

[12] A. Schindeler, M. M. McDonald, P. Bokko, and D. G. Little, "Bone remodeling during fracture repair: The cellular picture," in Seminars in Cell \& Developmental Biology, 2008, vol. 19, no. 5, pp. 459-466: Elsevier.

[13] B. Klosterhoff, S. Nagaraja, J. Dedania, R. Guldberg, and N. Willett, "Material and mechanobiological considerations for bone regeneration," in Materials for Bone Disorders: Elsevier, 2017, pp. 197-264.

[14] C. A. Malone, N. J. Sauer, and T. W. Fenton, "A radiographic assessment of pediatric fracture healing and time since injury," Journal of Forensic Sciences, vol. 56, no. 5, pp. 1123-1130, 2011.

[15] T. A. Einhorn, "The cell and molecular biology of fracture healing," Clinical Orthopaedics and Related Research, vol. 355, pp. S7-S21, 1998.

[16] L. Claes, N. Maurer-Klein, T. Henke, H. Gerngross, M. Melnyk, and P. Augat, "Moderate soft tissue trauma delays new bone formation only in the early phase of fracture healing," Journal of Orthopaedic Research, vol. 24, no. 6, pp. 1178-1185, 2006.

[17] L. Claes et al., "Monitoring and healing analysis of 100 tibial shaft fractures," Langenbeck's Archives of Surgery, vol. 387, no. 3-4, pp. 146-152, 2002.

[18] C. T. Brighton and R. M. Hunt, "Histochemical localization of calcium in the fracture callus with potassium pyroantimonate. Possible role of chondrocyte 
mitochondrial calcium in callus calcification," The Journal of Bone and Joint Surgery. American Volume, vol. 68, no. 5, pp. 703-715, 1986.

[19] J. Richardson, J. Cunningham, A. Goodship, B. O'connor, and J. Kenwright, "Measuring stiffness can define healing of tibial fractures," Bone \& Joint Journal, vol. 76, no. 3, pp. 389-394, 1994.

[20] J. Kenwright et al., "Axial movement and tibial fractures. A controlled randomised trial of treatment," Bone \& Joint Journal, vol. 73, no. 4, pp. 654-659, 1991.

[21] F. Burny, M. Donkerwolcke, R. Bourgois, M. Domb, and O. Saric, "Twenty years experience in fracture healing measurement with strain gauges," (in eng), Orthopedics, vol. 7, no. 12, pp. 1823-6, Dec 011984.

[22] M. Dreischarf et al., "In vivo implant forces acting on a vertebral body replacement during upper body flexion," (in eng), Journal of Biomechanics, vol. 48, no. 4, pp. 560-5, Feb 262015.

[23] G. Bergmann et al., "In vivo gleno-humeral joint loads during forward flexion and abduction," Journal of Biomechanics, vol. 44, pp. 1543-1552, 2011.

[24] P. Westerhoff et al., "In vivo measurement of shoulder joint loads during activities of daily living," Journal of Biomechanics, vol. 42, pp. 1840-1849, 2009.

[25] J. A. Szivek, R. F. Roberto, and D. S. Margolis, "In vivo strain measurements from hardware and lamina during spine fusion," Journal of Biomedical Materials Research, vol. 75B, no. 2, p. 8, 2005.

[26] P. J. Nicholls, E. Berg, J. F. Bliven, and J. M. Kling, "X-ray diagnosis of healing fractures in rabbits," Clinical Orthopaedics and Related Research, no. 142, pp. 234236, 1979. 
[27] F. Burny, "Study of consolidation of fractures by deformation gauges in clinical medicine," Acta Orthopaedica Belgica, vol. 34, no. 6, pp. 917-927, 1968.

[28] L. Claes and J. Cunningham, "Monitoring the mechanical properties of healing bone," Clinical Orthopaedics and Related Research, vol. 467, no. 8, pp. 1964-1971, 2009 .

[29] B. S. Klosterhoff et al., "Wireless implantable sensor for non-invasive, longitudinal quantification of axial strain across rodent long bone defects," Journal of Biomechanical Engineering, vol. 139, no. 11, p. 111004, 2017.

[30] B. S. Klosterhoff et al., "Implantable sensors for regenerative medicine," Journal of Biomechanical Engineering, vol. 139, no. 2, p. 021009, 2017.

[31] H. M. Frost, "Wolff's Law and bone's structural adaptations to mechanical usage: an overview for clinicians," The Angle Orthodontist, vol. 64, no. 3, pp. 175-188, 1994.

[32] L. Claes and C. Heigele, "Magnitudes of local stress and strain along bony surfaces predict the course and type of fracture healing," Journal of Biomechanics, vol. 32, no. 3, pp. 255-266, 1999.

[33] A. Goodship and J. Kenwright, "The influence of induced micromovement upon the healing of experimental tibial fractures," Bone \& Joint Journal, vol. 67, no. 4, pp. 650-655, 1985.

[34] G. Calori, E. Mazza, M. Colombo, C. Ripamonti, and L. Tagliabue, "Treatment of long bone non-unions with polytherapy: indications and clinical results," Injury, vol. 42, no. 6, pp. 587-590, 2011.

[35] Z. Huang, P.-G. Ren, T. Ma, R. L. Smith, and S. B. Goodman, "Modulating osteogenesis of mesenchymal stem cells by modifying growth factor availability," Cytokine, vol. 51, no. 3, pp. 305-310, 2010. 
[36] D. Lacroix, P. Prendergast, G. Li, and D. Marsh, "Biomechanical model to simulate tissue differentiation and bone regeneration: application to fracture healing," Medical and Biological Engineering and Computing, vol. 40, no. 1, pp. 14-21, 2002.

[37] D. Lacroix and P. Prendergast, "A mechano-regulation model for tissue differentiation during fracture healing: analysis of gap size and loading," Journal of Biomechanics, vol. 35, no. 9, pp. 1163-1171, 2002.

[38] P. Prendergast, R. Huiskes, and K. Søballe, "Biophysical stimuli on cells during tissue differentiation at implant interfaces," Journal of Biomechanics, vol. 30, no. 6, pp. 539-548, 1997.

[39] D. R. Carter, G. S. Beaupré, N. J. Giori, and J. A. Helms, "Mechanobiology of skeletal regeneration," Clinical Orthopaedics and Related Research, vol. 355, pp. S41-S55, 1998.

[40] P. Augat, U. Simon, A. Liedert, and L. Claes, "Mechanics and mechano-biology of fracture healing in normal and osteoporotic bone," Osteoporosis International, vol. 16, no. 2, pp. S36-S43, 2005.

[41] J. Boerckel, B. Uhrig, N. Willett, N. Huebsch, and R. Guldberg, "Mechanical regulation of vascular growth and tissue regeneration in vivo," Proceedings of the National Academy of Sciences of the United States of America, vol. 108, no. 37, pp. E674-80, 2011.

[42] J. D. Boerckel, Y. M. Kolambkar, H. Y. Stevens, A. S. Lin, K. M. Dupont, and R. E. Guldberg, "Effects of in vivo mechanical loading on large bone defect regeneration," Journal of Orthopaedic Research, vol. 30, no. 7, pp. 1067-1075, 2012.

[43] A. E. Goodship, T. J. Lawes, and C. T. Rubin, "Low-magnitude high-frequency mechanical signals accelerate and augment endochondral bone repair: Preliminary 
evidence of efficacy," Journal of Orthopaedic Research, vol. 27, no. 7, pp. 922930, 2009.

[44] T. P. Rüedi and W. M. Murphy, "AO principles of fracture management," Davos: AO Publishing \& Stuttgart New York: Georg Thieme Verlag, 2000.

[45] D. Marsh, "Concepts of fracture union, delayed union, and nonunion," Clinical Orthopaedics and Related Research, vol. 355, pp. S22-S30, 1998.

[46] A. Sarmiento, J. F. Schaeffer, L. Beckerman, L. L. Latta, and J. E. Enis, "Fracture healing in rat femora as affected by functional weight-bearing," The Journal of Bone and Joint Surgery. American Volume, vol. 59, no. 3, pp. 369-375, 1977.

[47] M. Tveit and J. Kärrholm, "Low effectiveness of prescribed partial weight bearing," Journal of Rehabilitation Medicine, vol. 33, no. 1, pp. 42-46, 2001.

[48] J. D. Boerckel, K. M. Dupont, Y. M. Kolambkar, A. S. Lin, and R. E. Guldberg, "In vivo model for evaluating the effects of mechanical stimulation on tissueengineered bone repair," Journal of Biomechanical Engineering, vol. 131, no. 8, p. 084502, 2009.

[49] P. Augat et al., "Early, full weightbearing with flexible fixation delays fracture healing," Clinical Orthopaedics and Related Research ${ }^{\circledR}$, vol. 328, pp. 194-202, 1996.

[50] C. Rubin, M. Bolander, J. P. Ryaby, and M. Hadjiargyrou, "The use of low-intensity ultrasound to accelerate the healing of fractures," Journal of Bone \& Joint Surgery, vol. 83 , no. 2 , p. $259,2001$.

[51] N. M. Pounder and A. J. Harrison, "Low intensity pulsed ultrasound for fracture healing: a review of the clinical evidence and the associated biological mechanism of action," Ultrasonics, vol. 48, no. 4, pp. 330-338, 2008. 
[52] L. R. Duarte, "The stimulation of bone growth by ultrasound," Archives of Orthopaedic and Traumatic Surgery, vol. 101, no. 3, pp. 153-159, 1983.

[53] R. Mundi, S. Petis, R. Kaloty, V. Shetty, and M. Bhandari, "Low-intensity pulsed ultrasound: Fracture healing," Indian Journal of Orthopaedics, vol. 43, no. 2, p. $132,2009$.

[54] K. S. Leung et al., "Low-magnitude high-frequency vibration accelerates callus formation, mineralization, and fracture healing in rats," Journal of Orthopaedic Research, vol. 27, no. 4, pp. 458-465, 2009.

[55] J. Wang, K. Leung, S. Chow, and W. Cheung, "The effect of whole body vibration on fracture healing-a systematic review," European Cells and Materials, vol. 34, pp. 108-127, 2017.

[56] H. K. Uhthoff, P. Poitras, and D. S. Backman, "Internal plate fixation of fractures: short history and recent developments," Journal of Orthopaedic Science, vol. 11, no. 2, pp. 118-126, 2006.

[57] S. M. Perren, "Evolution of the internal fixation of long bone fractures: the scientific basis of biological internal fixation: choosing a new balance between stability and biology," The Journal of Bone and Joint Surgery. British Volume, vol. 84, no. 8, pp. 1093-1110, 2002.

[58] G. A. Ilizarov, "The tension-stress effect on the genesis and growth of tissues. Part I. The influence of stability of fixation and soft-tissue preservation," Clinical Orthopaedics and Related Research, no. 238, pp. 249-281, 1989.

[59] J. Curie and P. Curie, "Développement par compression de l'électricité polaire dans les cristaux hémièdres à faces inclinées," Bulletin de Minéralogie, vol. 3, no. 4, pp. 90-93, 1880. 
[60] G. Lippmann, "Principe de la conservation de l'électricité, ou second principe de la théorie des phénomènes électriques," Journal de Physique Théorique et Appliquée, vol. 10, no. 1, pp. 381-394, 1881.

[61] S.-T. Ho and S.-J. Jan, "A piezoelectric motor for precision positioning applications," Precision Engineering, vol. 43, pp. 285-293, 2016.

[62] L. Howald, H. Rudin, and H. J. Güntherodt, "Piezoelectric inertial stepping motor with spherical rotor," Review of Scientific Instruments, vol. 63, no. 8, pp. 39093912, 1992.

[63] W. Robbins, D. Polla, and D. Glumac, "High-displacement piezoelectric actuator utilizing a meander-line geometry I. Experimental characterization," IEEE Transactions on Ultrasonics, Ferroelectrics, and Frequency Control, vol. 38, no. 5, pp. 454-460, 1991.

[64] T. Sharma, S.-S. Je, B. Gill, and J. X. Zhang, "Patterning piezoelectric thin film PVDF-TrFE based pressure sensor for catheter application," Sensors and Actuators A: physical, vol. 177, pp. 87-92, 2012.

[65] E. S. Kim and R. S. Muller, "IC-processed piezoelectric microphone," IEEE Electron Device Letters, vol. 8, no. 10, pp. 467-468, 1987.

[66] H. Gullapalli et al., "Flexible piezoelectric $\mathrm{ZnO}$-paper nanocomposite strain sensor," Small, vol. 6, no. 15, pp. 1641-1646, 2010.

[67] J. J. Dosch, D. J. Inman, and E. Garcia, "A self-sensing piezoelectric actuator for collocated control," Journal of Intelligent Material Systems and Structures, vol. 3 , no. 1, pp. 166-185, 1992.

[68] T. Furukawa, K. Ishida, and E. Fukada, "Piezoelectric properties in the composite systems of polymers and PZT ceramics," Journal of Applied Physics, vol. 50, no. 7, pp. 4904-4912, 1979. 
[69] IEEE, "ANSI/IEEE Std 176-1987," 1987.

[70] J. Sirohi and I. Chopra, "Fundamental understanding of piezoelectric strain sensors," Journal of Intelligent Material Systems and Structures, vol. 11, no. 4, pp. 246-257, 2000.

[71] ThorLabs, "Piezo Actuators," 2019.

[72] H. Jung and D.-G. Gweon, "Creep characteristics of piezoelectric actuators," Review of Scientific Instruments, vol. 71, no. 4, pp. 1896-1900, 2000.

[73] D. Damjanovic, "Hysteresis in piezoelectric and ferroelectric materials," The Science of Hysteresis, vol. 3, pp. 337-465, 2006.

[74] J. G. Smits, "Influence of moving domain walls and jumping lattice defects on complex material coefficients of piezoelectrics," IEEE Transactions on Sonics and Ultrasonics, vol. 23, no. 3, pp. 168-173, 1976.

[75] G. Arlt, "Piezoelectric relaxation," Ferroelectrics, vol. 40, no. 1, pp. 149-157, 1982.

[76] T. Kleckers, "Strain Gage and Piezoelectric Based Force Sensors - A Comparison," in SENSOR, Nürnberg, Germany, 2011, vol. B3, pp. 257-260.

[77] Bluetooth SIG, "Bluetooth Market Update 2019," 2019.

[78] Silicon Labs, "BLE113 Bluetooth Smart Module," Datasheet 2015.

[79] W. Dargie and C. Poellabauer, Fundamentals of wireless sensor networks: theory and practice. John Wiley \& Sons, 2010.

[80] Bluetooth Core Specifications Version 4.0, 2010.

[81] B. S. Klosterhoff et al., "Real-time monitoring of mechanical cues in the regenerative niche reveal dynamic strain magnitudes that enhance bone repair," Manuscript submitted for publication, 2019. 
[82] A. Fleming and S. Moheimani, "Improved current and charge amplifiers for driving piezoelectric loads, and issues in signal processing design for synthesis of shunt damping circuits," Journal of Intelligent Material Systems and Structures, vol. 15, no. 2, pp. 77-92, 2004.

[83] A. Fleming and S. Moheimani, "Precision current and charge amplifiers for driving highly capacitive piezoelectric loads," Electronics Letters, vol. 39, no. 3, pp. 282284, 2003.

[84] K. Goto, T. Nakagawa, O. Nakamura, and S. Kawata, "An implantable power supply with an optically rechargeable lithium battery," IEEE Transactions on Biomedical Engineering, vol. 48, no. 7, pp. 830-833, 2001.

$[85]$

Woodbank Communications. (2005). Available: https://www.mpoweruk.com/performance.htm

[86] S. A. Sapareto and W. C. Dewey, "Thermal dose determination in cancer therapy," International Journal of Radiation Oncology, Biology, Physics, vol. 10, no. 6, pp. 787-800, 1984.

[87] G. C. Van Rhoon, T. Samaras, P. S. Yarmolenko, M. W. Dewhirst, E. Neufeld, and N. Kuster, "CEM $43^{\circ} \mathrm{C}$ thermal dose thresholds: a potential guide for magnetic resonance radiofrequency exposure levels?," European Radiology, vol. 23, no. 8, pp. 2215-2227, 2013.

[88] Dymax, "1072-M Product Data Sheet."

[89] R. J. Halter et al., "The correlation of in vivo and ex vivo tissue dielectric properties to validate electromagnetic breast imaging: initial clinical experience," Physiological measurement, vol. 30, no. 6, p. S121, 2009. 
[90] T. Wehner, U. Wolfram, T. Henzler, F. Niemeyer, L. Claes, and U. Simon, "Internal forces and moments in the femur of the rat during gait," Journal of Biomechanics, vol. 43, no. 13, pp. 2473-2479, 2010. 


\section{A PCB Layout}
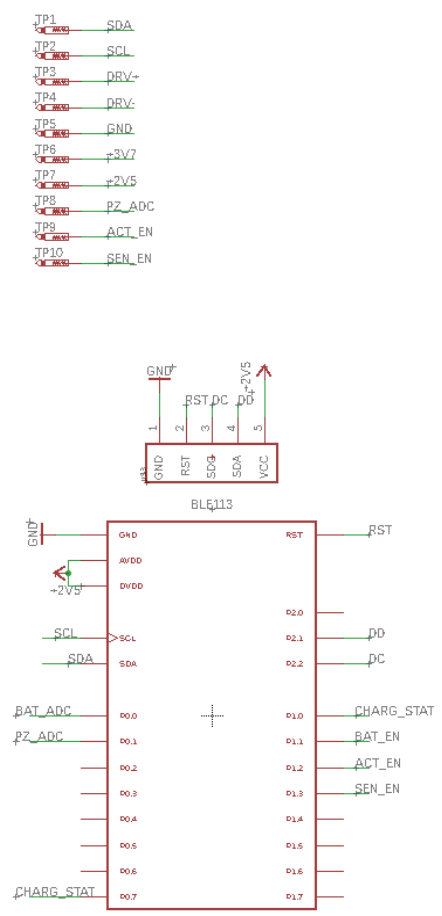
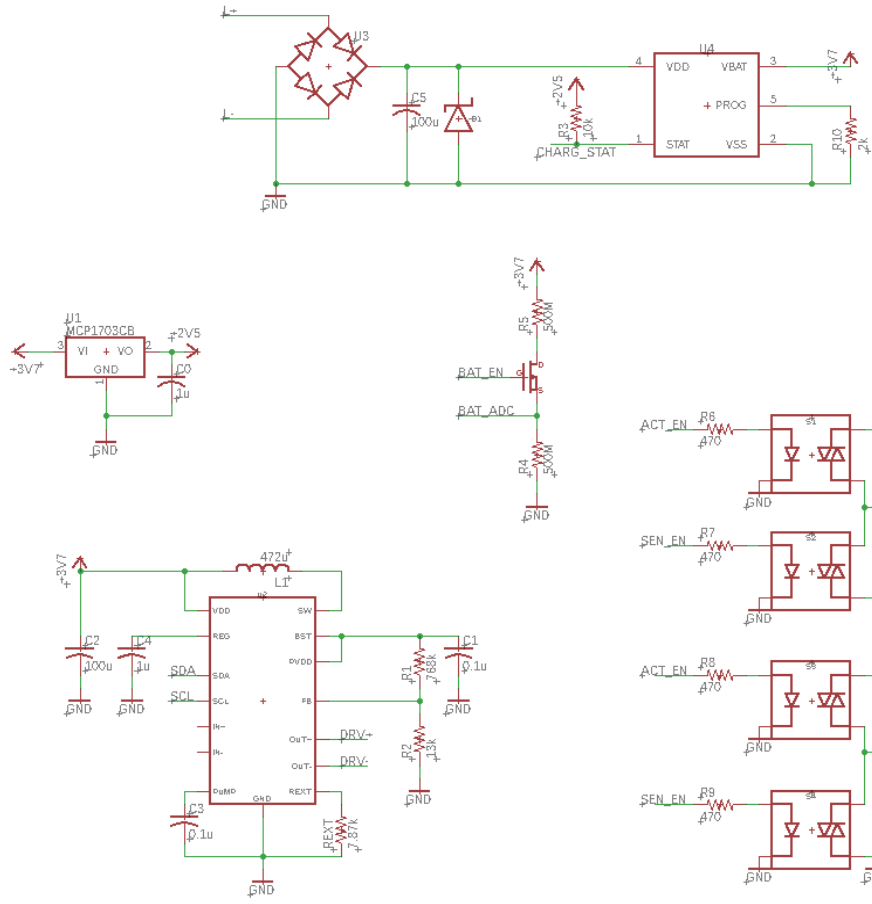
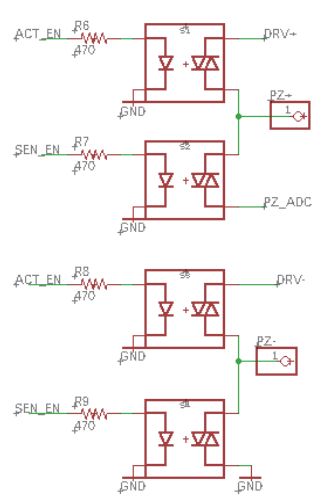
B

PCB Layout

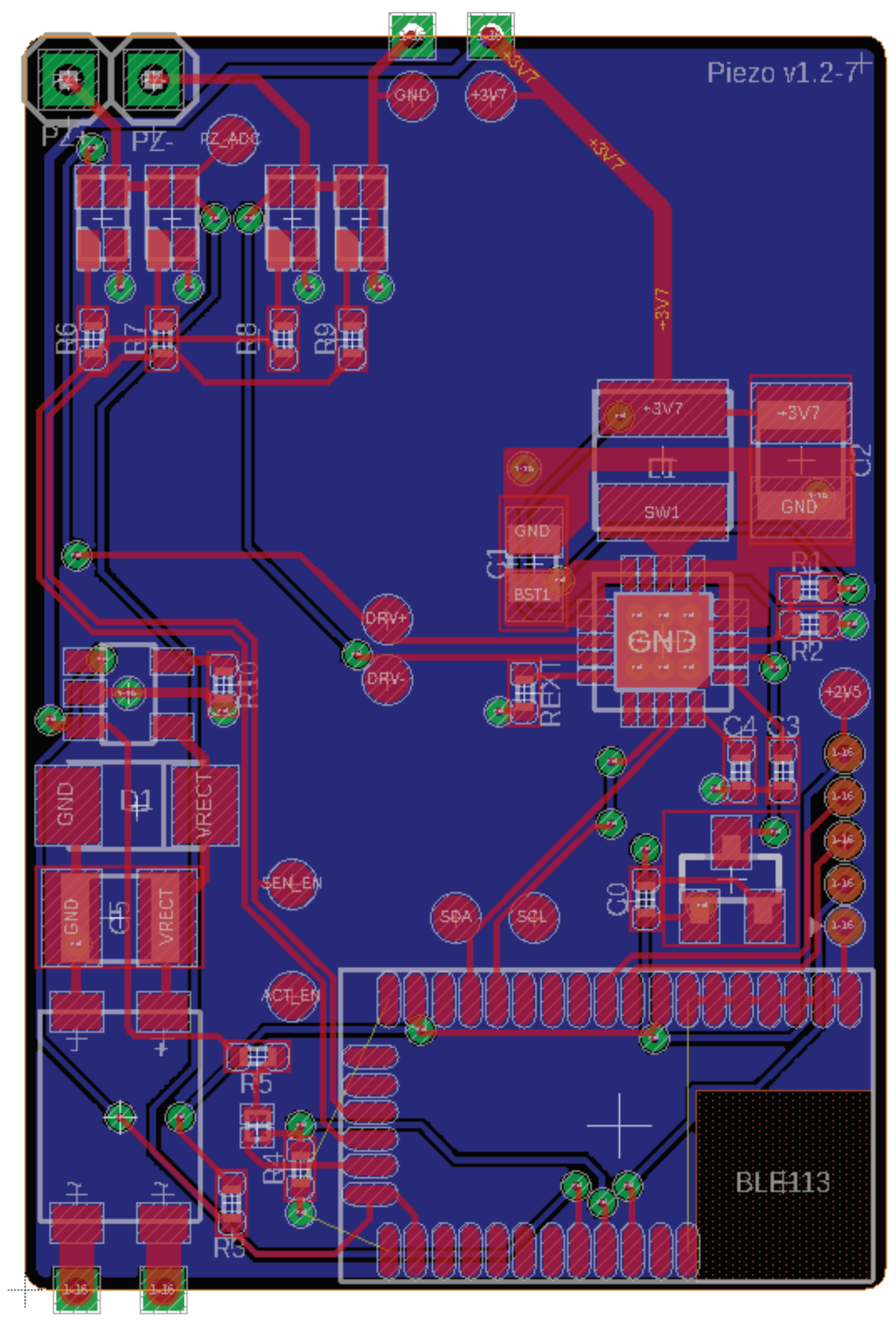




\section{Telemetry Software}

\section{C.1 Main File}

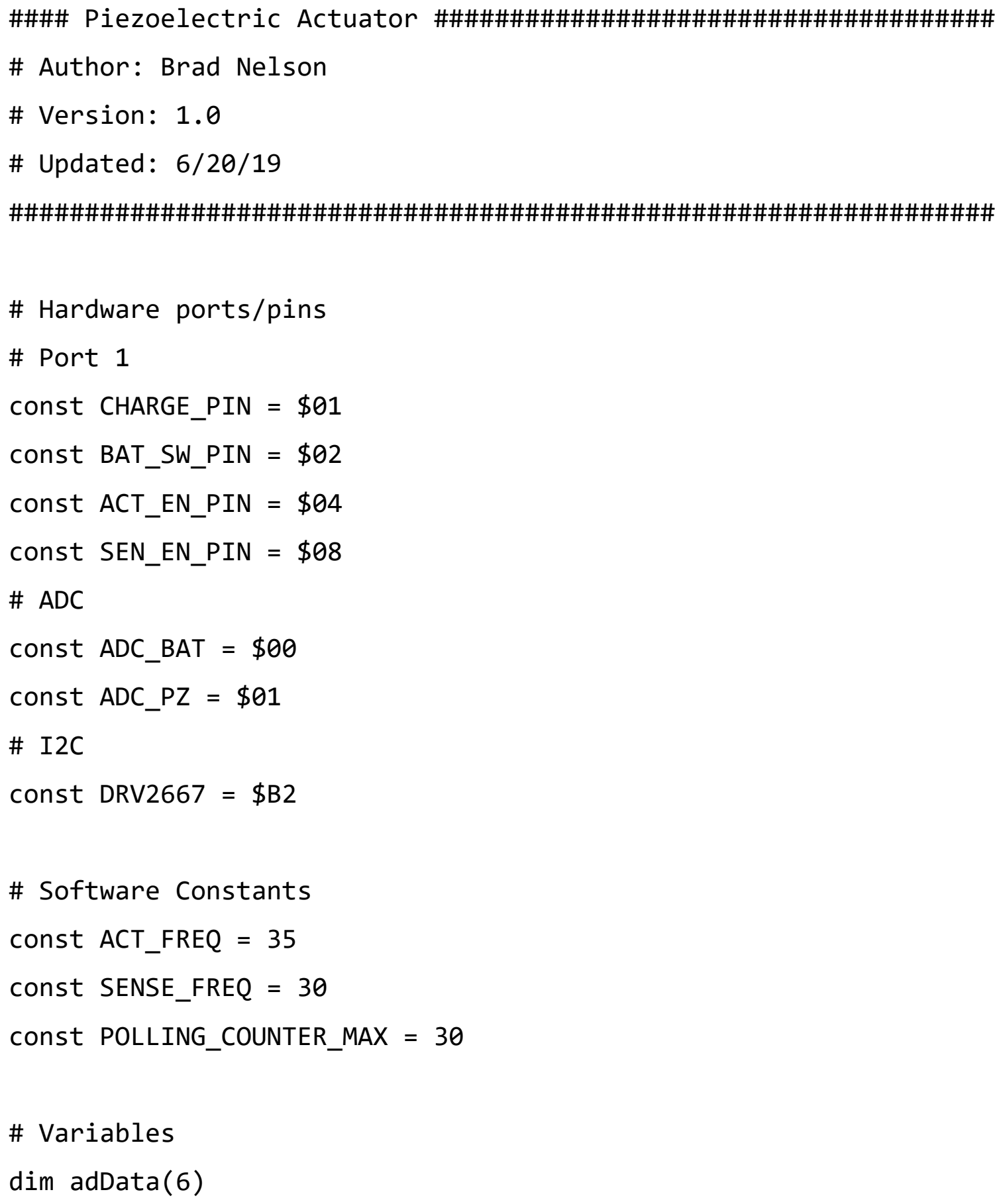




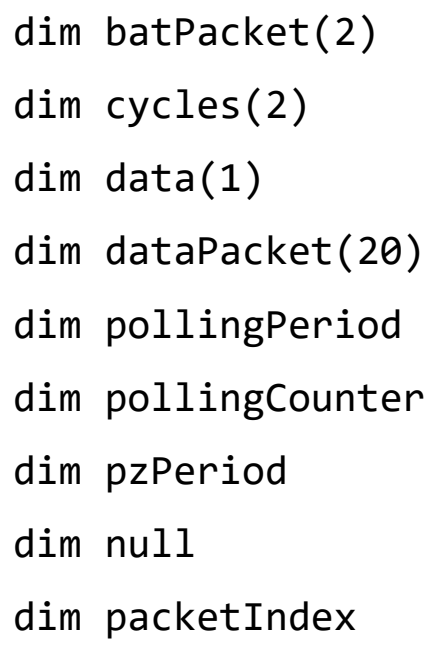

\# Define number of cycles 
$\operatorname{cycles}(\theta: 1)=" \backslash x \theta 8 "$

$\operatorname{cycles}(1: 1)=$ duration

\# Control

call hardware_i2c_write(DRV2667,1,2, "\x02\x00")

call hardware_i2c_write(DRV2667,1, 2, "\x01\x00")

call hardware_i2c_write(DRV2667,1,2, "\x03\x01")

call hardware_i2c_write(DRV2667,1,2,"\x04\x00")

\# Header

call hardware_i2c_write(DRV2667,1,2, "\xFF \x01")

call hardware_i2c_write(DRV2667,1, 2, "\x00\x05")

call hardware_i2c_write(DRV2667,1, 2, "\x01\x80")

call hardware_i2c_write(DRV2667,1,2, "\x02\x06")

call hardware_i2c_write(DRV2667,1,2, "\x03\x00")

call hardware_i2c_write(DRV2667, 1, 2, "\x04\x09")

call hardware_i2c_write(DRV2667,1,2,"\x05\x9D") \# Run for $5 \mathrm{sec}$ \# Data

call hardware_i2c_write(DRV2667,1,2, "\x06\xCC")

call hardware_i2c_write(DRV2667,1,2," \x07\x04")

call hardware_i2c_write(DRV2667,1,2, cycles $(\theta: 2)$ )

call hardware_i2c_write(DRV2667,1,2,"\x09\x00")

\# Control

call hardware_i2c_write(DRV2667,1,2," \xFF \x00")

call hardware_i2c_write(DRV2667,1,2,"\x02\x01")

end

procedure set_ad_data(charging)

\# Discoverable/Connectable

$\operatorname{adData}(\theta: 1)=2$ 


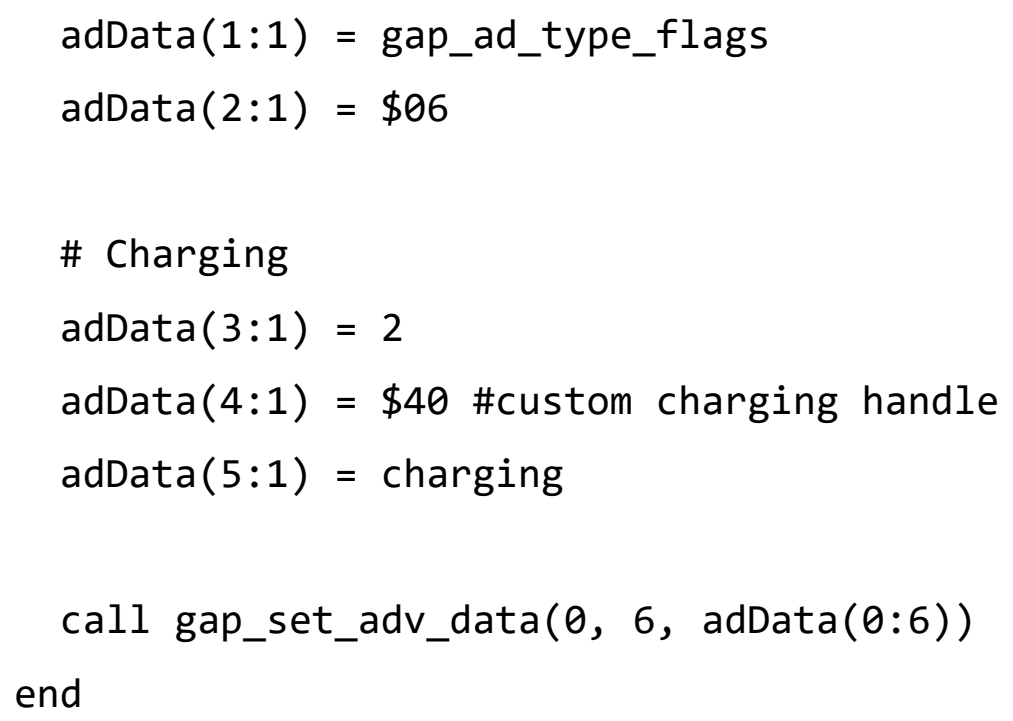


call hardware_io_port_write $(1, \$ 0 \mathrm{E}, \$ 01)$

end

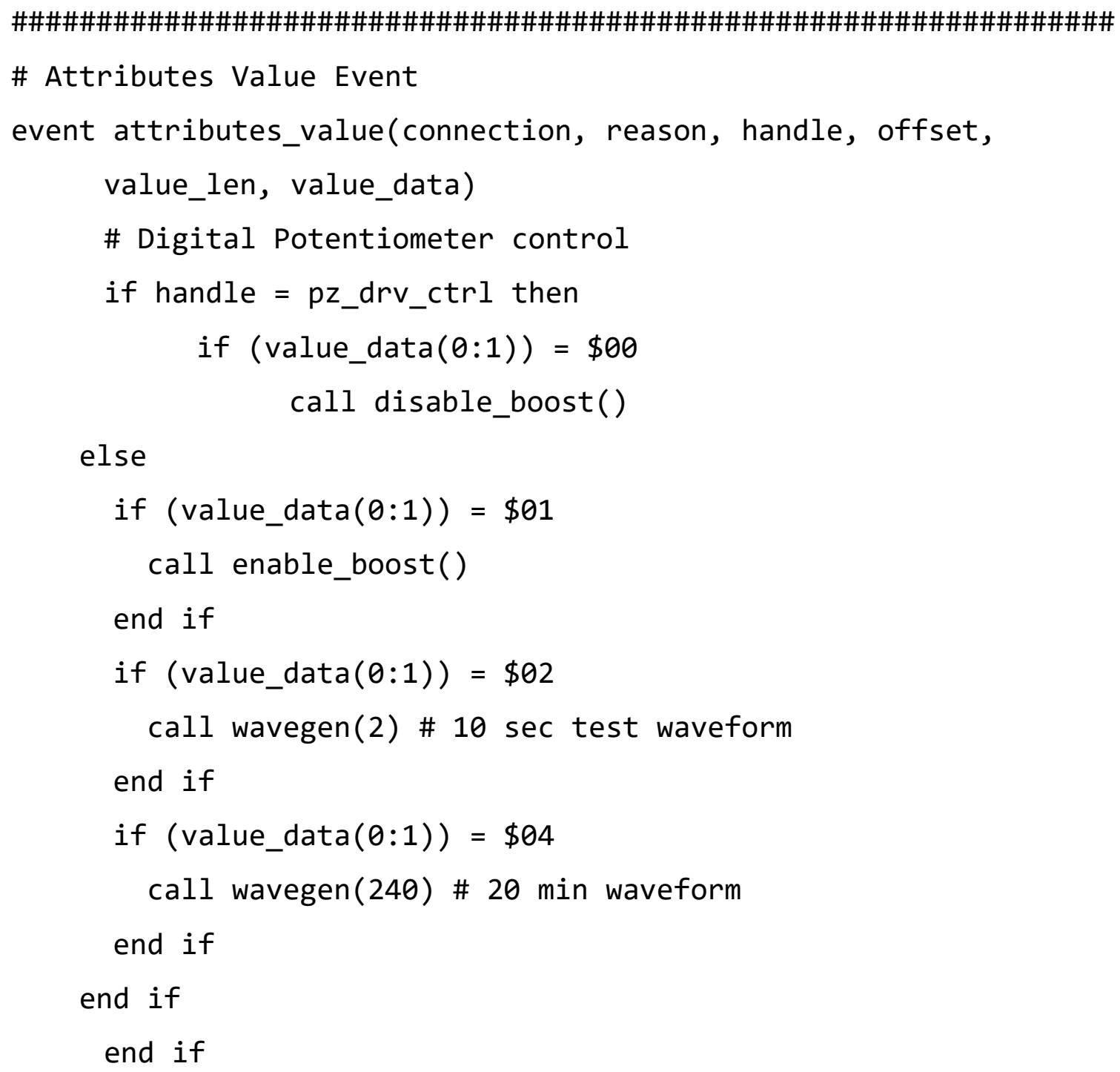


call hardware_io_port_write( 1, ACT_EN_PIN + SEN_EN_PIN, $\$ 00)$

call hardware_set_soft_timer $(0,0,1)$

end if

\# 1 - Actuation mode

if (value_data $(0: 1))=\$ 01$

call hardware_io_port_write( 1, ACT_EN_PIN +

SEN_EN_PIN, ACT_EN_PIN)

call hardware_set_soft_timer $(0,0,1)$

end if

\# 2 - Sensing mode

if (value_data $(0: 1))=\$ 02$

packetIndex $=0$

call hardware_io_port_write( 1, ACT_EN_PIN +

SEN_EN_PIN, SEN_EN_PIN)

call hardware_set_soft_timer(pzPeriod, 0, 1)

end if

end if

end

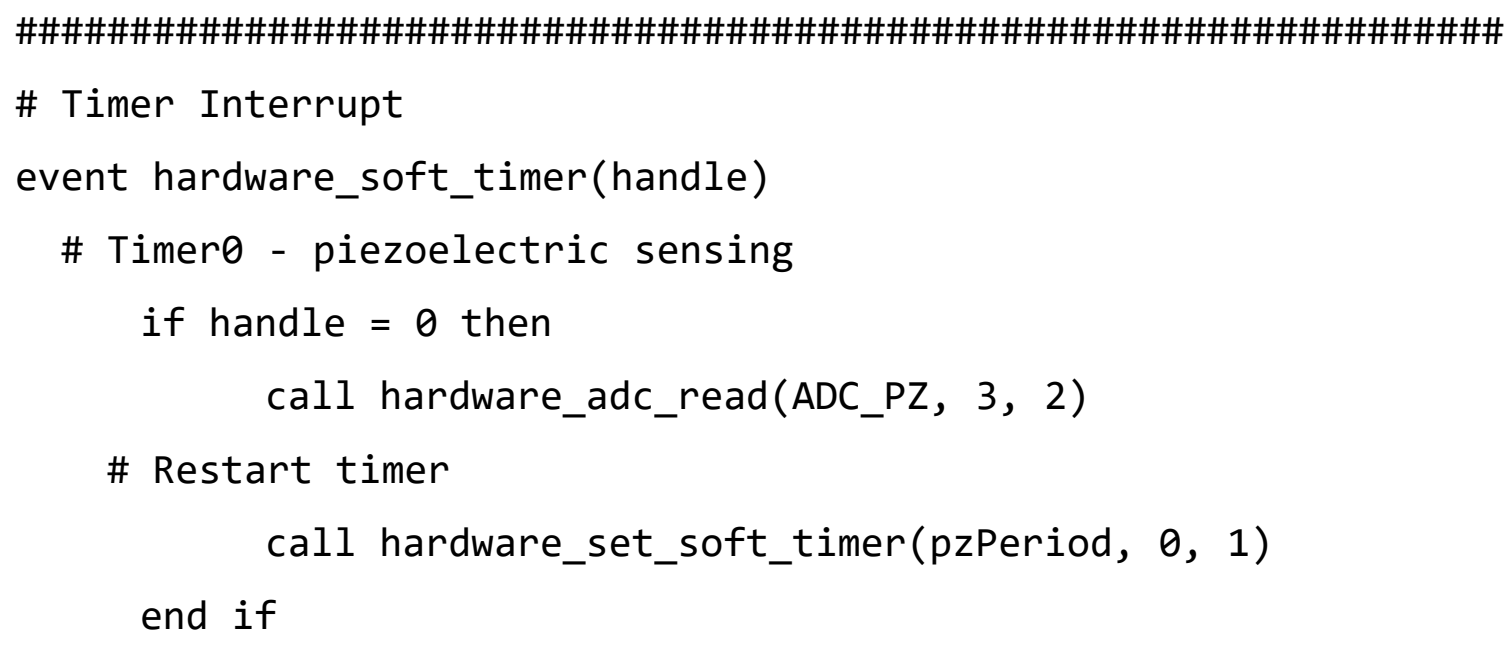




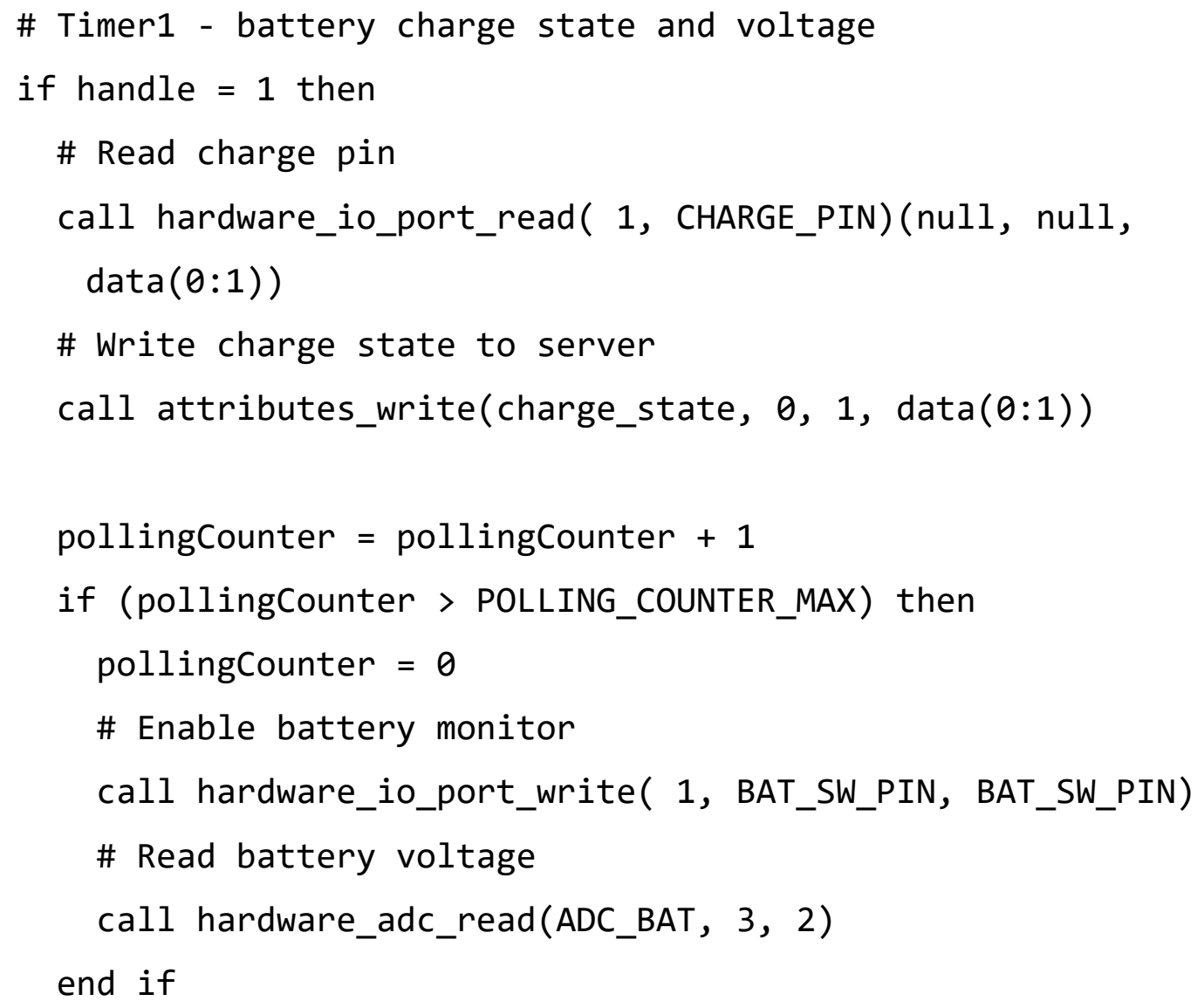




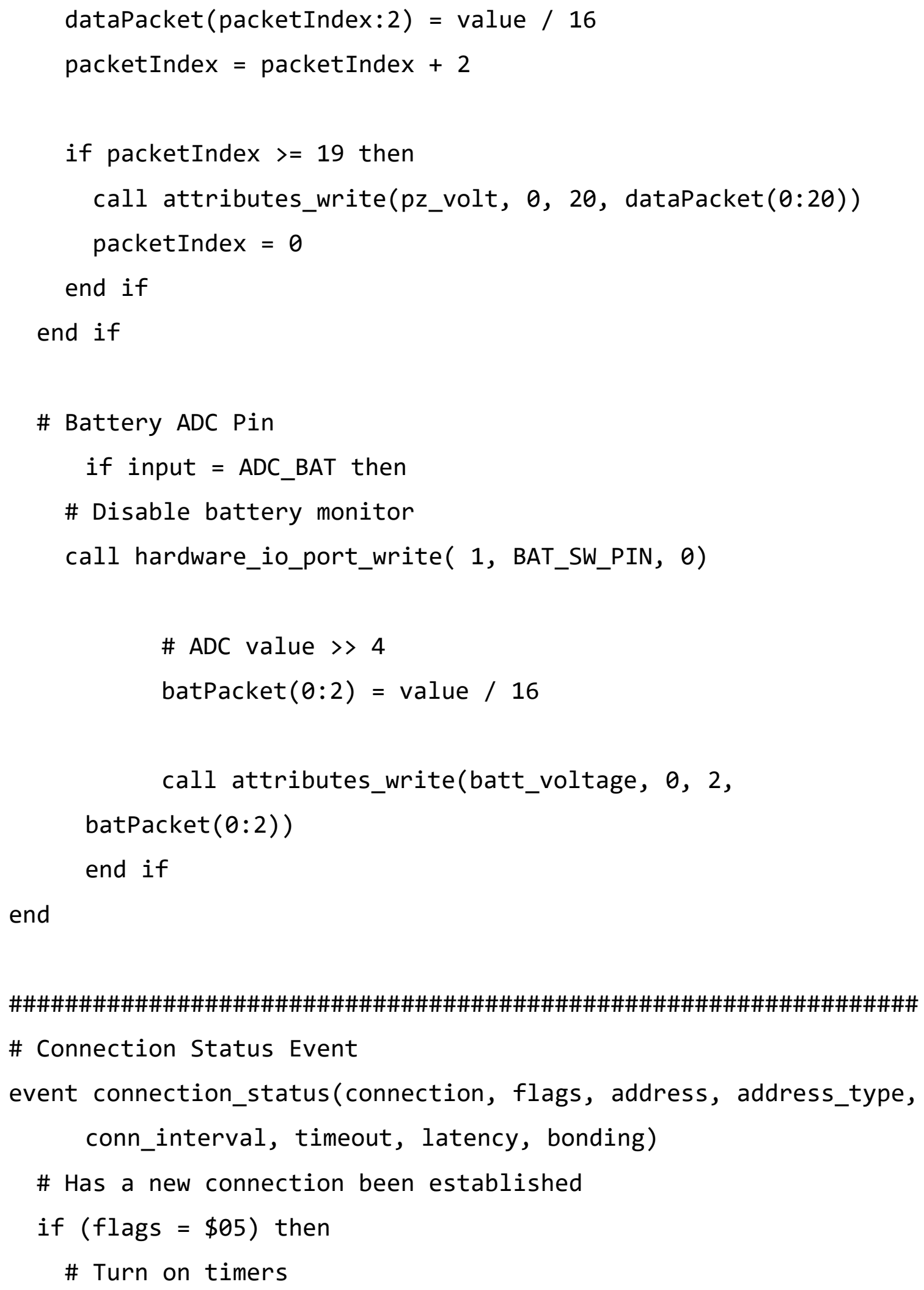




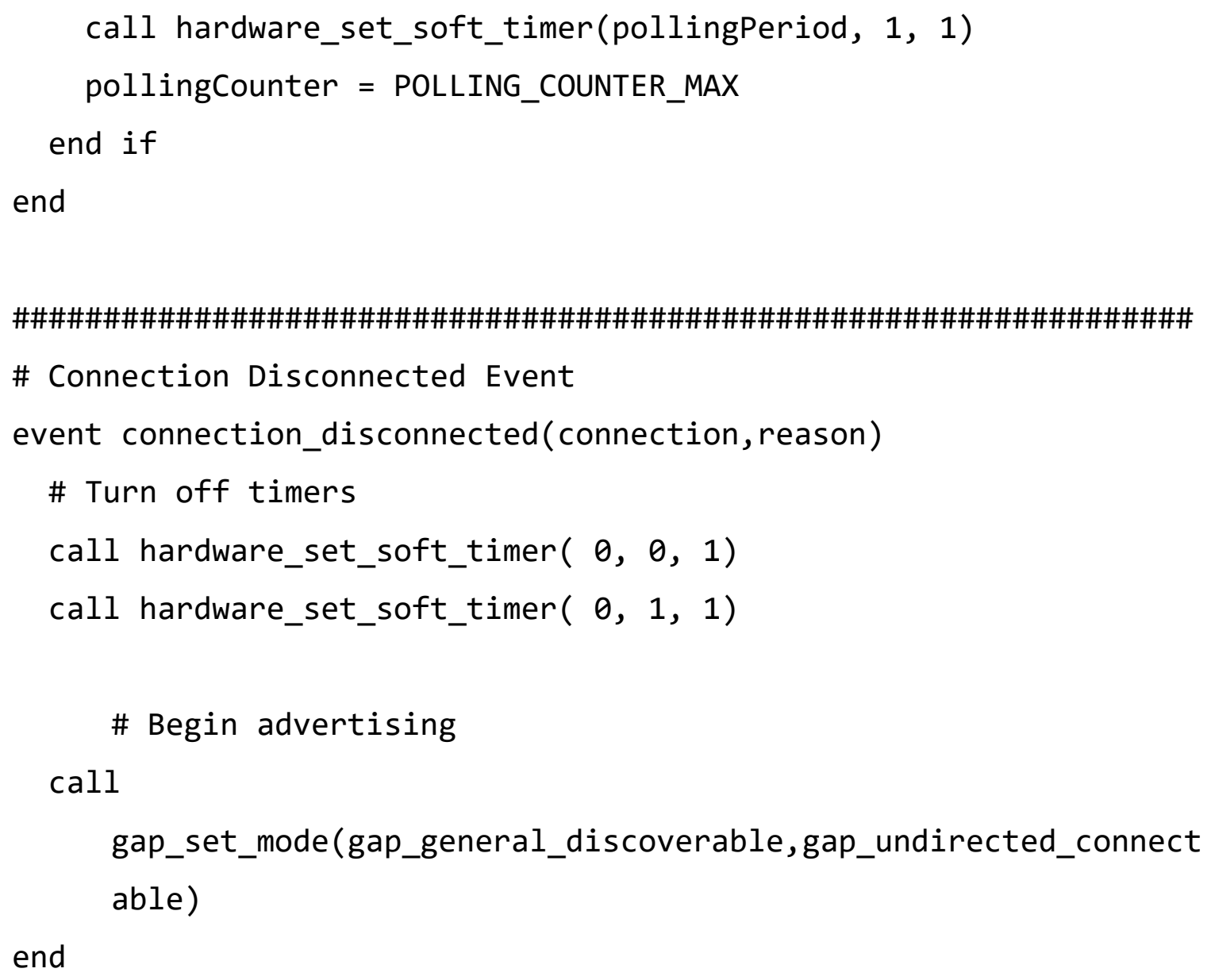

\section{C.2 GATT File}

<?xml version="1.0" encoding="UTF-8" ?>

<configuration>

<service uuid="1800">

<description>Generic Access Profile</description>

〈characteristic uuid="2a00">

<description>Device Name</description>

<properties read="true" const="true" />

$\langle$ value $>$ Z1</value $>$

$\langle/$ characteristic $>$ 


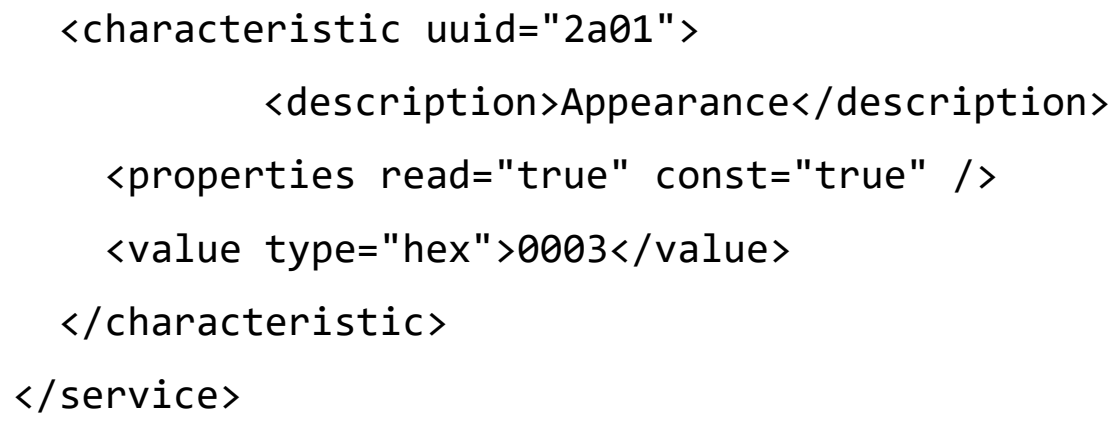


$\langle/$ service $>$

<service uuid="a451a318-a828-484f-9011-5f8accbf6480" advertise="true">

<description>MTU Biosensors Service</description> <characteristic uuid="77da051e-8378-11e7-bb31-

be2e44b06b34" id="gatt_debug">

<description>Debug Packet</description> <properties read="true" write="true"/> <value length="2" type="hex"/> $\langle/$ characteristic $>$

$\langle/$ service $>$

<!-- Battery Service-->

<service uuid="763b50b2-75a4-11e9-8f9e-2a86e4085a59" advertise="true"> <characteristic uuid="7d79ef76-7335-11e9-a923-1681be663d3e" id="charge_state"> <properties notify="true" read="true"/> <value length="1">1</value>

$\langle/$ characteristic > <characteristic uuid="7d79ef76-7335-11e9-a923-1681be663d3f" id="batt_voltage"> <properties notify="true" read="true" write="false"/> <value length="2" type="hex" >0</value $\rangle$

$\langle/$ characteristic $>$ <characteristic uuid="741e32a6-70a4-11e7-857b-a6006ad3dba1" id="batt_temp">

<description>Temperature Packet</description> 


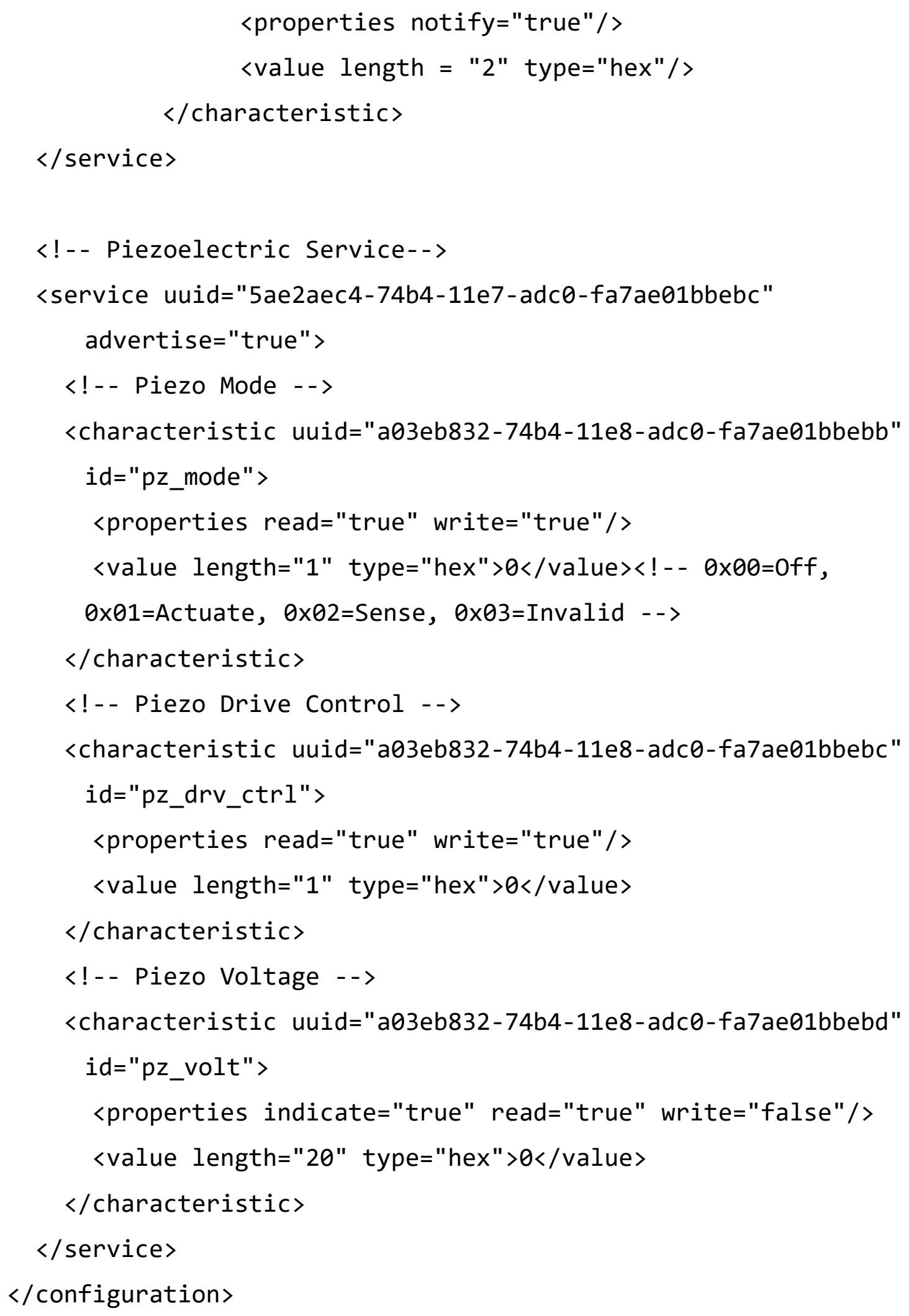




\section{Copyright Information}

The following figures use content licensed under Creative Commons license 3.0:

Figure 1.1: "Fracture Repair" by Servier Medical Art, Licensed under Creative Commons 3.0 - https://smart.servier.com/smart image/bone-fracture-13/, https://smart.servier.com/smart image/bone-fracture-14/, https://smart.servier.com/smart image/bone-fracture-15/, https://smart.servier.com/smart image/bone-fracture-16/. Accessed July 2019.

Figure 1.4: "Femur" by Servier Medical Art, Licensed under Creative Commons 3.0 https://smart.servier.com/smart_image/femur/. Accessed July 2019. 